\title{
WestVirginiaUniversity
}

THE RESEARCH REPOSITORY @ WVU

Graduate Theses, Dissertations, and Problem Reports

2006

\section{Enhanced iris recognition: Algorithms for segmentation, matching and synthesis}

Samir Shah

West Virginia University

Follow this and additional works at: https://researchrepository.wvu.edu/etd

\section{Recommended Citation}

Shah, Samir, "Enhanced iris recognition: Algorithms for segmentation, matching and synthesis" (2006). Graduate Theses, Dissertations, and Problem Reports. 2481.

https://researchrepository.wvu.edu/etd/2481

This Thesis is protected by copyright and/or related rights. It has been brought to you by the The Research Repository @ WVU with permission from the rights-holder(s). You are free to use this Thesis in any way that is permitted by the copyright and related rights legislation that applies to your use. For other uses you must obtain permission from the rights-holder(s) directly, unless additional rights are indicated by a Creative Commons license in the record and/ or on the work itself. This Thesis has been accepted for inclusion in WVU Graduate Theses, Dissertations, and Problem Reports collection by an authorized administrator of The Research Repository @ WVU. For more information, please contact researchrepository@mail.wvu.edu. 


\title{
Enhanced Iris Recognition: Algorithms for Segmentation, Matching and Synthesis
}

\author{
by \\ Samir Shah \\ Thesis submitted to the \\ College of Engineering and Mineral Resources \\ at West Virginia University \\ in partial fulfillment of the requirements \\ for the degree of
}

Master of Science

in

Electrical Engineering

Arun A. Ross, Ph.D., Chair

Xin Li, Ph.D.

Lawrence Hornak, Ph.D.

Lane Department of Computer Science and Electrical Engineering

\author{
Morgantown, West Virginia \\ 2006
}

Keywords: Iris recognition, Iris synthesis, Feature agglomeration, Markov Random Field (MRF), Cubic splines, Geodesic Active Contours (GAC), Level sets, Snakes, Iris segmentation, Iriscodes, Multi-unit fusion, Multi-instance fusion. 


\author{
Abstract \\ Enhanced Iris Recognition: Algorithms for Segmentation, Matching and Synthesis \\ by \\ Samir Shah \\ Master of Science in Electrical Engineering \\ West Virginia University \\ Arun A. Ross, Ph.D., Chair
}

This thesis addresses the issues of segmentation, matching, fusion and synthesis in the context of irises and makes a four-fold contribution. The first contribution of this thesis is a post matching algorithm that observes the structure of the differences in feature templates to enhance recognition accuracy. The significance of the scheme is its robustness to inaccuracies in the iris segmentation process. Experimental results on the CASIA database indicate the efficacy of the proposed technique. The second contribution of this thesis is a novel iris segmentation scheme that employs Geodesic Active Contours to extract the iris from the surrounding structures. The proposed scheme elicits the iris texture in an iterative fashion depending upon both the local and global conditions of the image. The performance of an iris recognition algorithm on both the WVU non-ideal and CASIA iris database is observed to improve upon application of the proposed segmentation algorithm. The third contribution of this thesis is the fusion of multiple instances of the same iris and multiple iris units of the eye, i.e., the left and right iris at the match score level. Using simple sum rule, it is demonstrated that both multi-instance and multi-unit fusion of iris can lead to a significant improvement in matching accuracy. The final contribution is a technique to create a large database of digital renditions of iris images that can be used to evaluate the performance of iris recognition algorithms. This scheme is implemented in two stages. In the first stage, a Markov Random Field model is used to generate a background texture representing the global iris appearance. In the next stage a variety of iris features, viz., radial and concentric furrows, collarette and crypts, are generated and embedded in the texture field. Experimental results confirm the validity of the synthetic irises generated using this technique. 
I dedicate my thesis to my family 


\section{Acknowledgments}

Last two years have been the most exciting years in my life. I want to take this opportunity and thank all the people who have made my stay at WVU most memorable.

I am highly obliged to Dr. Arun Ross for being my advisor and the committee chair. His devotion to work, extremely hardworking nature, a very positive attitude has motivated me a lot. The last one year when I worked with him, I gained so much knowledge that I can say it got the best out of me.

I am very grateful to my committee members Dr. Xin Li and Dr. Lawrence Hornak for their valuable guidance and suggestions for my thesis. Their comments have been very useful in enhancing the presentation of this thesis. Also, I was fortunate to have an opportunity to learn Dr. McGraw's course in medical image analysis which helped me significantly in my thesis.

I would also like to extend my gratitude to all lab-mates Simona, Shigefumi, Chris, Sarvesh, Kiran, Kelly, Matt, Phani, Rohan, Rajiv, Nikhil and Dong who have been with me all the time and for their co-operation and necessary feedback. I would also like to thank my friends Raviraj, Manoday, Shantanu, Rajas, Sanket and all in WVU to be always with me and I would never forget the fun I had with all of them in Morgantown.

I would like to thank my parents and my sisters for their support, never-fading love and sacrifice. It was because of them I could survive hard times and be consistent on my work. Lastly, I would also like to thank my wife, Jidnya, for standing by me and sharing my joys and frustrations. Without her, I would not have come so far. 


\section{Contents}

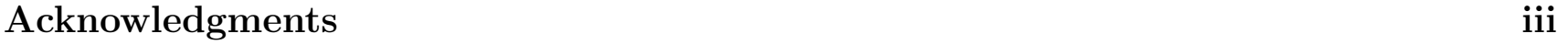

List of Figures $\quad$ vi

$\begin{array}{ll}\text { List of Tables } & \text { ix }\end{array}$

1 Introduction $\quad 1$

1.1 Biometrics . . . . . . . . . . . . . . . . . . . . 1

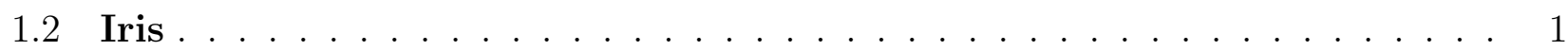

1.3 Iris recognition $\ldots \ldots \ldots \ldots \ldots \ldots \ldots \ldots$

1.3.1 Appearance-based methods . . . . . . . . . . . . . . . 4

1.3 .2 Filter-based methods . . . . . . . . . . . . . . . . . . . . . . . . 4

1.3.3 Feature-based methods . . . . . . . . . . . . . . . . . 6

1.4 Challenges in iris recognition . . . . . . . . . . . . . . . . . 7

1.5 Contributions of this thesis . . . . . . . . . . . . . . . . . . . 7

2 Iris Segmentation using Geodesic Active Contours $\quad 10$

2.1 Motivation . . . . . . . . . . . . . . . . . . 10

2.2 Baseline iris segmentation, encoding and matching algorithm . . . . . . . 11

2.3 Enhanced matching utilizing the structure of differences in iriscodes . . 13

2.4 Iris Segmentation using Geodesic Active Contours . . . . . . . . . . . . . . 17

2.4 .1 Pupil Segmentation . . . . . . . . . . . . . . . . . . . 17

2.4 .2 Iris segmentation . . . . . . . . . . . . . . . . . . . . . . . . . . . . . . . . . . . . . .

Geodesic Active Contours . . . . . . . . . . . . . . . . . . . . . 21

Iris segmentation using Geodesic Active Contours . . . . . . . . . . 25

2.5 Analyzing segmentation performance . . . . . . . . . . . . . 33

2.5.1 A rejection criteria for highly occluded irises . . . . . . . . . . . . . 34

2.5.2 Using GAC to segment other iris dataset images . . . . . . . . . . . 36

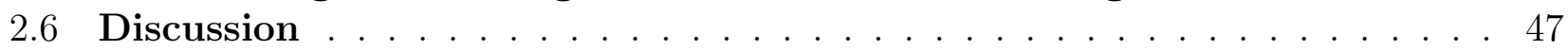

3 Multi-Instance, Multi-Unit Fusion $\quad 49$

3.1 Limitations of using single iris image . . . . . . . . . . . . . . . . . . . 49

3.2 Fusion in iris systems . . . . . . . . . . . . . . . . . . . . . . . . . 51

3.3 Fusion in other biometric systems . . . . . . . . . . . . . . . . . . . . . 52

3.4 Multi-instance iris fusion . . . . . . . . . . . . . . . . . . . . . . 53

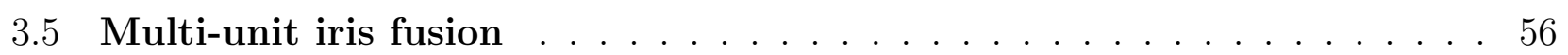

3.6 Discussion . . . . . . . . . . . . . . . . . . . . . 61 
4 Iris Synthesis using Feature Agglomeration $\quad 62$

4.1 Iris synthesis . . . . . . . . . . . . . . . . . . . . . . . . 62

4.2 Feature agglomeration . . . . . . . . . . . . . . . . . 64

$4.2 .1 \quad$ Texture synthesis using Markov Random Field . . . . . . . . . . . . 64

Synthesis using single primitive . . . . . . . . . . . . . . . 65

4.2 .2 Feature synthesis . . . . . . . . . . . . . . . . . 66

4.3 Results of synthesis . . . . . . . . . . . . . . . . . . . . . . . . . 69

$4.3 .1 \quad$ Clustering of iris images . . . . . . . . . . . . . . . 71

4.3 .2 Comparison of genuine and impostor distributions . . . . . . . . . 72

4.4 Discussion . . . . . . . . . . . . . . . . . . . . . . 74

$\begin{array}{llr}5 & \text { Summary and Future work } & 78\end{array}$

\begin{tabular}{lr}
\hline Appendices & 80
\end{tabular}

\begin{tabular}{|ll}
\hline A Iris Recognition based on Demographic Attributes & 80
\end{tabular}

A.1 Introduction . . . . . . . . . . . . . . . . . . . . . . 80

A.2 Experimental results . . . . . . . . . . . . . . . . . . . . . 81

A.3 Summary . . . . . . . . . . . . . . . . . . . . . . . . . . . 83

$\begin{array}{lr}\text { References } & 84\end{array}$ 


\section{List of Figures}

1.1 Anatomy of iris. The two white blobs in the pupil are the specular reflections due to the imaging device. . . . . . . . . . . . . . . . . . 2

1.2 Block diagram of a typical iris recognition system. . . . . . . . . . . . . . . . 3

2.1 Real part of 2-D Gabor wavelet filters. . . . . . . . . . . . . . . . . . . . 12

2.2 The genuine and impostor match score distribution indicating the region of overlap. 13

2.3 Irises incorrectly segmented using the integro-differential operators. . . . . . . . . 15

2.4 The block diagram summarizing the new matching scheme utilizing the blockwise differences in iriscodes. Here $a$ and $b$ are the boundaries of the overlap region between genuine and impostor scores. . . . . . . . . . . . . . . . . . 16

2.5 ROC curves indicating the performance improvement on the CASIA database using the structure of differences in iriscodes. . . . . . . . . . . . . . . 17

2.6 Pupil binarization. (a) Image of an eye with dark eyelashes; (b) Thresholded binary iris image. . . . . . . . . . . . . . . . . 17

2.7 2-D Median filtered binary iris image. . . . . . . . . . . . . . . . . 18

2.8 Traced boundaries of all the remaining objects in the binary image (shown in gray). 18

2.9 Fitting circle on all potential regions where pupil might be present (shown in gray). 19

2.10 Pupil segmentation. (a) Image of an eye with dark eyelashes; (b) Segmented pupil (shown using white color). . . . . . . . . . . . . . . . . 20

2.11 Pupil segmentation without inpainting of specular reflections. (a) Iris image; (b) Smoothed image; (c) Thresholded binary image; (d) 2-D Median filtered image; (e) Under-segmented pupil (shown using white color). . . . . . . . . . . . . 20

2.12 Pupil segmentation using inpainting of specular reflections in the vicinity of the pupil. (a) Iris image; (b) Smoothed image; (c) Inpainted specular reflection in the vicinity of the pupil; (d) Thresholded binary image; (e) 2-D Median filtered image; (f) Segmented pupil (shown using white color). . . . . . . . . . . . . . 21

2.13 Pupil segmentation. (a) Iris image; (b) Thresholded binary image; (c) 2-D Median filtered image; (d) Segmented pupil (shown using white color). . . . . . . . . . . 22

2.14 Curve $\gamma$ evolving towards the boundary of the object. . . . . . . . . . . . . . . 23

2.15 Stopping function for the geodesic active contours.(a) Example iris image from CASIA user 5; (b) Stopping function $K$; (c) Modified stopping function $K^{\prime}$. . . . . 26

2.16 Contour initialization for iris segmentation.(a) Example iris image from CASIA user 5; (b) Zero level set (initial contour); (c) Mesh plot of the signed distance

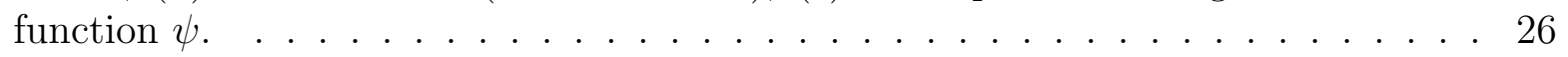


2.17 Evolution of the geodesic active contour during iris segmentation.(a) Iris image from CASIA user 5 with initial contour; (b) Embedding function $\psi$ (X and $\mathrm{Y}$ axis correspond to the size of the iris image and the $\mathrm{Z}$ axis represents different level sets); (c), (d), (e), (f), (g), (h), (i), (j), (k), (l) Contours after every 100 iterations and their corresponding embedding functions; (m) Final contour after 700 iterations (contours shown using white color). . . . . . . . . . . . .

2.18 Effect of minimizing the thin plate energy of the levels sets on the geodesic active contour evolution.(a) Contour without minimizing the think plate energy of the level sets; (b) Contour obtained by minimizing the thin plate energy of the level sets (contours shown using white color).

2.19 Final contour segmenting the iris.(a) Geodesic contour splitting at various local minima; (b) Final contour (contours shown using white color). . . . . . . . . . . .

2.20 Robust detection of the radius and co-ordinates of the iris using geodesic active contours. (a) and (b) Radius of the iris estimated using circular gradient operators; (c) and (d) Radius of the iris estimated using geodesic active contours (iris radius shown using white color). .

2.21 Perona-Malik anisotropic diffusion.(a) Normalized iris image; (b) Normalized iris image enhanced using histogram equalization; (c) Normalized iris image enhanced using anisotropic diffusion and histogram equalization. . . . . . . . . . . . . . 33

2.22 Receiver Operating Characteristics for the CASIA iris database. . . . . . . . . . . 34

2.23 Receiver Operating Characteristics for the WVU non-ideal iris database: (a) Left iris; (b) Right iris. .

2.24 Occluded irises due to eyelids and eyelashes in the CASIA dataset. (a) Occluded iris (user 41); (b) Occluded iris (user 91). . . . . . . . . . . . . . . 36

2.25 Masks of the occluded irises in Figure 2.24 due to eyelids and eyelashes. . . . . . . 36

2.26 Histogram of available iris texture information in the normalized images of CASIA. 37

2.27 ROC curves indicating the performance improvement when iris images (11\%) with very large occlusion are rejected. . . . . . . . . . . . . . . . . . 37

2.28 Sample irises rejected using the available iris texture information criterion. . . . . 38

2.29 Masks of irises rejected in Figure 2.28 using the available iris texture information criterion.

2.30 Histogram of available iris texture information in the normalized images of the WVU non-ideal iris database: (a) Right iris; (b) Left iris. . . . . . . . . . . . . . .

2.31 ROC curves indicating the performance improvement when iris images $(2 \%$ of right irises and $3 \%$ of left irises of WVU non-ideal iris database) with very large occlusion are rejected: (a) Right iris; (b) Left iris. . . . . . . . . . . . . . . . . . .

2.32 Receiver Operating Characteristics for the WVU off-angle iris database: (a) Right iris; (b) Left iris. . . . . . . . . . . . . . . . . . . . . . 42

2.33 Segmenting WVU Non-Ideal irises using the proposed algorithm. . . . . . . . . . . 43

2.34 Segmenting UBIRIS irises using the proposed algorithm. . . . . . . . . . . . . . . 44

2.35 Segmenting $M M U 1$ irises using the proposed algorithm. . . . . . . . . . . . . . 45

3.1 Common problems encountered during the image acquisition process. . . . . . . . 50

3.2 An iris image showing a contact lens having the characters "AV" imprinted on it. 51

3.3 Information fusion in irises: (a) Multi-instance fusion; (b) Multi-unit fusion. . . . . 54

3.4 Performance of iris recognition using single enrolled impressions $\left(I_{1}\right.$ and $\left.I_{2}\right)$ of left and right irises of WVU non-ideal iris database: (a) Right iris; (b) Left iris. . . . . 55 
3.5 Performance of iris recognition using single enrolled impressions $\left(I_{1}\right.$ and $\left.I_{2}\right)$ of CASIA iris database. . . . . . . . . . . . . . . . . . . 56

3.6 Performance improvement of iris recognition by fusing two impressions of the WVU left and right irises using the simple sum rule: (a) Right iris; (b) Left iris. . 57

3.7 Performance improvement of iris recognition by fusing two irises of CASIA iris database using the simple sum rule. . . . . . . . . . . . . . . . . 58

3.8 Performance improvement of iris recognition using multi-unit fusion of left and right irises using sum rule: (a) Fusing impressions $I_{1}$ from both irises; (b) Fusing impressions $I_{2}$ from both irises. . . . . . . . . . . . . . . . . . . 59

3.9 Performance improvement of iris recognition using multi-unit-instance fusion of both the $I_{1}$ and $I_{2}$ impressions of the left and right irises using the sum rule . . .

4.1 Selection of primitive from a real iris image. (a) An iris image from the CASIA database; (b) The associated unwrapped image; (c) Primitive extracted from the unwrapped image. . . . . . . . . . . . . . . . . . . 64

4.2 Block diagram summarizing texture synthesis using MRF. . . . . . . . . . . . . 65

4.3 Texture synthesis using MRF. (a) Sample primitive from real iris image; (b) Bigger synthesized texture image; (c) Carved out circular iris; (d) Iris with radial gradient. 66

4.4 Synthesis of radial interlaced furrows. (a) Linear furrows; (b) Deformed furrows using periodic cubic splines. . . . . . . . . . . . . . . . 67

4.5 Lending texture to a streamline using LIC. (a) White noise image, T; (b) Convolving T with a streamline; (c) Result of LIC. . . . . . . . . . . . . . 67

4.6 Synthesis of collarette. (a) Interlaced furrows ; (b) collarette shape generated using periodic cubic splines ; (c) Radial furrows circumscribed by collarette; (d) Synthesized iris with eyelids and eyelashes. . . . . . . . . . . . . . 68

4.7 Synthesis of concentric furrows. (a) Synthesized iris with concentric furrows; (b) Synthesized iris with eyelids and eyelashes. . . . . . . . . . . . . . 68

4.8 Synthesis of crypts. (a) A synthesized shape of crypt; (b) ROI for averaging; (c) Multiple crypts; (d) Synthesized iris with crypts; (e) Synthesized iris with eyelids and eyelashes.

4.9 Examples of two synthesized iris classes with seven images per class. (a) Synthesized iris; (b) Pupil size decreased by 10 pixels; (c) Pupil size increased by 10 pixels; (d) Smoothed synthesized iris; (e) Synthesized iris added with noise; (f) Rotated synthesized iris (-10 degrees); (g) Rotated synthesized iris (10 degrees). . 70

4.10 Impostor distributions for real and synthetic images. . . . . . . . . . . . . . 73

4.11 Genuine and impostor match score distributions of real and synthetic images. . . . 73

4.12 Examples of synthesized iris images of different classes. . . . . . . . . . . . . 75

4.13 Graphical user interface. . . . . . . . . . . . . . . . . . . . . 77

A.1 Performance of iris recognition for subjects belonging to different ethnicity. . . . . 81

A.2 Performance of iris recognition for subjects having different eye color. . . . . . . . 82

A.3 Performance of iris recognition for subjects belonging to different sex. . . . . . . . 82 


\section{List of Tables}

1.1 Examples of a few iris segmentation, encoding and matching techniques proposed in the literature . . . . . . . . . . . . . . . . . . . . . 8

2.1 The GAR at a fixed FAR using Masek's code, integro-differential operators for iris segmentation and statistical analysis of iriscodes. . . . . . . . . . . . . . 15

2.2 The GAR at a fixed FAR for the CASIA iris database using Masek's code, integrodifferential operators and geodesic active contours (GAC) for iris segmentation. . . 46

2.3 The GAR at a fixed FAR for the WVU non-ideal right-iris database using Masek's code, integro-differential operators and geodesic active contours (GAC) for iris segmentation. . . . . . . . . . . . . . . . . . . 46

2.4 The GAR at a fixed FAR for the WVU non-ideal left-iris database using Masek's code, integro-differential operators and geodesic active contours (GAC) for iris

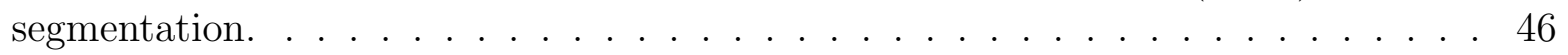

2.5 Parameters in iris segmentation using Geodesic Active Contours. . . . . . . . . . . 48

3.1 The GAR at a fixed FAR for single impressions $I_{1}$ and $I_{2}$, multi-instance fusion $\left(I_{1}+I_{2}\right)$, multi-unit fusion $\left(I_{1}\right.$ of left $+I_{1}$ of right and similarly $I_{2}$ of left $+I_{2}$ of right) and multi-instance-multi-unit fusion. . . . . . . . . . . . . 6 61

$4.1 \quad$ Examples of publicly available iris databases. . . . . . . . . . . . . . . . . . 63

4.2 Confusion matrix indicating iris and non-iris classification. . . . . . . . . . . . . 72 


\section{Chapter 1}

\section{Introduction}

\subsection{Biometrics}

Biometrics refers to the use of automated or semi-automated methods for recognizing humans based on their physical or behavioral traits. The physical traits include fingerprints, face, iris, retina, voice, hand geometry, finger vein, palm vein, sclera veins, etc. Signature, gait, keystroke dynamics, etc. are examples of behavioral characteristics. A biometric system is a pattern recognition system that uses biometric identifiers to establish the identity of a person [1]. The basic purpose of biometrics is to define the identity of a person based on "who she is" rather than by "what she possesses" (e.g., an ID card) or "what she remembers" (e.g., a password). The focus of this thesis is on the iris biometric.

\subsection{Iris}

The iris is a vastly detailed texture. Its formation is independent on the genetic genotype and depends on the initial condition embryonic precursor to the iris. Thus, the iris texture is chaotic and unique to every individual. The iris is considered to be one of the most accurate and reliable biometric trait for the purpose of verification and identification of individuals [2]. The iris is an internal organ of the eye that is located just behind the cornea and in front of the lens. The functionality of the iris is to control the size of the pupil, which in turn regulates the amount of light entering the pupil and impinging on the retina. The visible structures around the iris are the pupil, sclera and cornea. The pupil lies near the center of the iris. It appears 
dark because most of the light entering the pupil is absorbed by the tissues inside it. The sclera is commonly known as "the white of the eye". It is the eye's protective outer cover. The cornea is the transparent part of the human eye which covers the iris, pupil and the anterior part of the human eye. The iris is a annulus structure consisting of fibrovascular tissues called stroma. It is divided into two regions, viz., pupillary zone which is the inner region of the iris on the periphery of the pupil and the ciliary zone which constitutes the rest of the iris as shown in Figure 1.1. These two regions are separated by the collarette which typically represents the thickest part of the human iris. Furrows found in the stroma on the circumference of the iris have a circular structure. They are termed as circular furrows. The majority of the furrows in the stroma are interlaced and radiate towards the pupil (dilator muscles) and are termed as radial furrows. Some circular furrows (sphincter muscles) are present just outside the pupil in a narrow band of about $1 \mathrm{~mm}$. The stroma connects the sphincter muscles, which contract the pupil, with the dilator muscles, which dilate the pupil. The iris also contains sharply demarcated crypts that are a result of iris thinning which exposes the darkly pigmented posterior layer. Thus, the iris in the presence of near-infrared lighting, is observed to have several features including radial and concentric furrows, crypts and the collarette, all of which contribute to its uniqueness and play a significant role in identifying an individual.

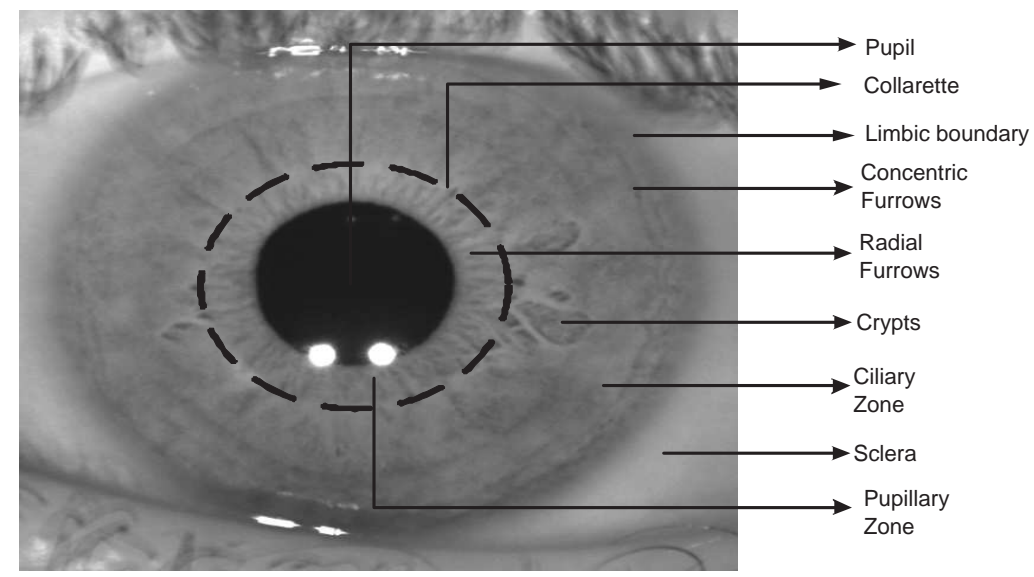

Figure 1.1: Anatomy of iris. The two white blobs in the pupil are the specular reflections due to the imaging device. 


\subsection{Iris recognition}

Flom et al. [3] postulate that the textural content of the iris is stable throughout an individual's lifespan. They state that every iris is unique and no two individuals (even those possessing the same genetic genotype) have similar irises. Indeed, the two irises of an individual have been observed to be different in their intricate detail. Hence, the iris is considered to be a robust and unique biometric with a very low False Accept Rate (FAR). The function of an iris recognition system is to extract, represent and compare the textural intricacy present on the surface of the iris. Such a system comprises of modules for iris segmentation, enhancement, feature extraction (encoding) and feature matching. During enrolment, the extracted feature templates are stored in the database. During authentication, a digital photograph of the iris is taken and the feature set extracted. This feature set is then matched with the templates in the database in order to determine or validate an individual's identity. Figure 1.2 shows the block diagram of a typical iris recognition system.

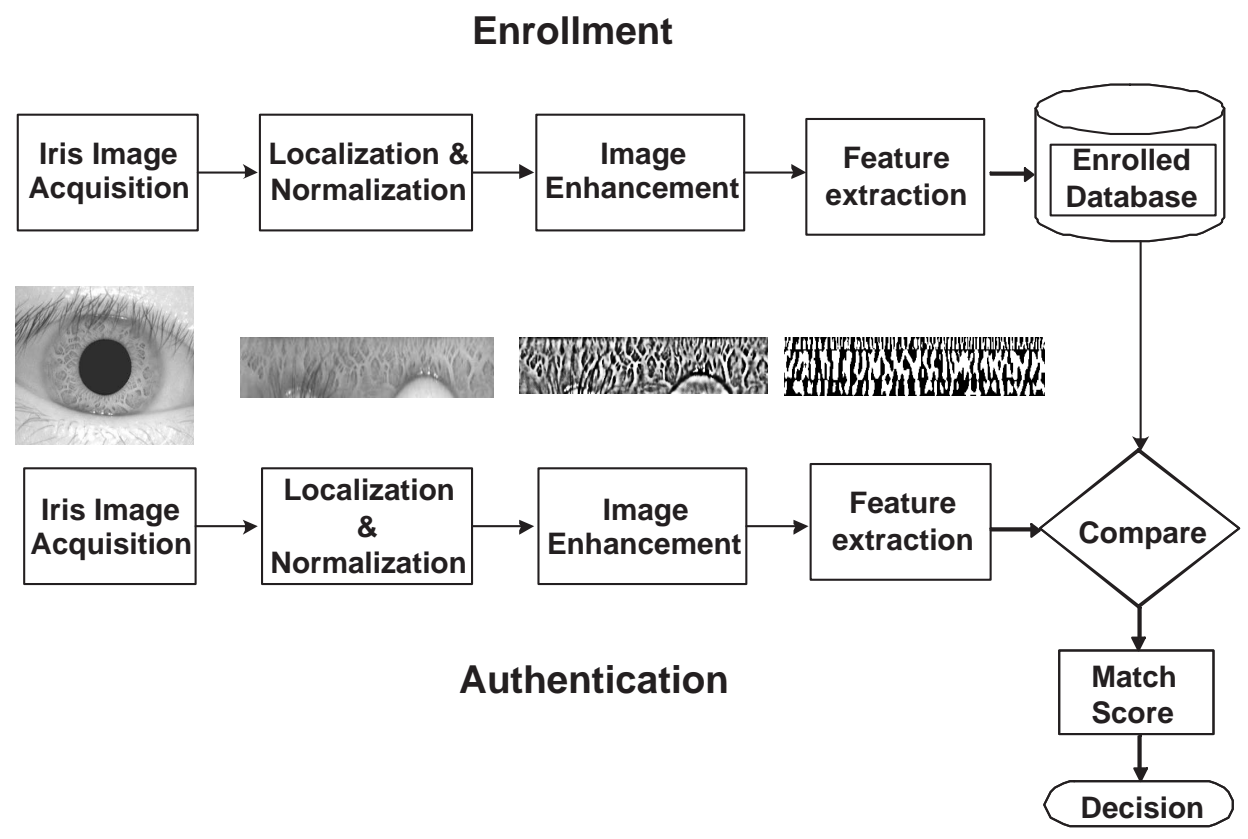

Figure 1.2: Block diagram of a typical iris recognition system.

Large-scale authentication experiments ( [4], [2], [5], [6]) have confirmed the notion that the iris texture is unique, further underscoring the relevance of this biometric trait in distinguishing individuals. Daugman implemented the world's first iris recognition system [4] for which he has 
the patent [7, the rights of which are now held by Iridian Technologies 1 . He uses the integrodifferential operator, which acts as a circular edge detector, for determining the inner and outer boundaries of the iris as well as the upper and lower eyelids. Multiscale 2-D Gabor-Wavelet coefficients are then used to encode the iris resulting in an iris template called an "iriscode". At the time of identification, the iriscode is matched with the iriscodes in the database. Over the years, several different algorithms have been presented in the literature. These algorithms can be classified into three broad different categories, viz., (a) appearance-based, (b) filter-based and (c) feature-based, depending on the method by which the iris texture is represented for matching purposes. Appearance-based methods use the pixel values of the iris (e.g. Principal component analysis (PCA) and Independent component analysis (ICA)) to represent the underlying iris images. Filter-based methods use certain filters like Gabor-Wavelet or Discrete cosine transform (DCT) to extract the textural properties of the iris texture. Feature-based techniques use the local features present in the iris like the crypts or fibers and their locations to represent various iris images. Examples for each of these categories is presented below.

\subsubsection{Appearance-based methods}

Noh et al. [8] use a feature extraction algorithm based on Independent Component Analysis (ICA). ICA is first used to generate a set of optimal basis vectors. Each iris image is then projected onto this set of basis vectors. The coefficients of the projections are used as feature vectors. The feature vectors are then converted to an iriscode by quantizing them. Dorairaj et al. 9] use a combination of Principal Component Analysis with Independent Component Analysis to encode the iris image. They use the integro-differential operators to localize the iris and the pupil. The matching is accomplished using both the Euclidean and the Hamming distance metrics.

\subsubsection{Filter-based methods}

Filter-based methods for encoding iris information are the most researched techniques in the iris recognition literature. The first iris recognition algorithm proposed by Daugman ( [4], [10], [2]) uses a texture-based method to encode irises. Multi-scale 2-D Gabor-Wavelet transform is used to generate a 256-byte 'iriscode'. Hamming distance is then used as a measure to determine

\footnotetext{
${ }^{1}$ Iridian Technologies http://www.iridiantech.com/
} 
the proximity of two iriscodes. The integro-differential operator, which acts as a circular edge detector, is employed for determining the inner and outer boundaries of the iris as well as the upper and lower eyelids.

Wildes ( [11], [5]) uses the Laplacian-of-a-Gaussian (LOG) filter to extract features from the iris image. A Hough transform-based method is used to segment the iris. Also, the upper and lower boundaries of the eyelid are approximated using parabolic curves. Matching is done using the normalized correlation between the test and training images.

Ma et al. ( [12], [13]) use a Hough transform based technique to determine the center and radius of the pupil and the iris. A bank of multi-scale Gaussian filters modulated by circularly symmetric sinusoidal function are used to produce discriminating texture features in irises. The filter response obtained on the image not only entails information from every orientation but also in both the $x$ and the $y$ directions. The filtered image is divided into small blocks and the mean and the average absolute deviation of each filtered block are used as the features.

Boles and Boashash [14] use the zero-crossings of the wavelet transform at various resolution levels over concentric circles on the iris as the feature vectors. Dissimilarity functions are used to match these feature vectors. Lim et al. [15] use 2-D four-level Haar wavelet transform to extract features from the iris image. The feature vector is formed by combining all the filtered pixel values of the high-pass filter of the $4^{\text {th }}$ level and the mean of the filtered pixel values of the remaining high-pass filters of the other three levels. The iris image is finally represented by quantizing the feature vector. A competitive learning neural network is used during matching to recognize the irises. Abhyankar and Schuckers [16] use training based Active Shape Models to segment the iris from the sclera. The non-linear shape of the iris is learned using a few training images. The normalized iris is then encoded by employing bi-orthogonal wavelet implemented using the lifting scheme [17].

Masek and Kovesi [18] employ weighted gradients using a combination of Kovesi's modified canny edge detector [19] and the circular Hough-transform to segment the iris. The normalized iris image is then convolved with 1-D Log-Gabor filters so that the DC component of the evensymmetric Gabor filter becomes zero. The iris is segmented using edge detection and Hough transform approach. Huang et al. [20] use edge detection and Hough transform based method to segment the irises. A bank of Log-Gabor filters are then used to capture local orientation characteristics of the iris so as to produce discriminating features. They later modified their 
iris segmentation algorithm [21] by proposing a new noise-removing approach by the use of edge information based on phase congruency. Yuan and Shi [22] segment the iris using edge detection and Hough transform. They filter the iris image using a 2-D log-Gabor filter with different scales and orientations. The phase congruency is computed by combining data over these scales and orientations. The candidate iris features are then compared with those stored in the database using the Euclidean distance metric.

Thornton et al. [23] use the Hough transform for iris segmentation. Advanced distortiontolerant correlation filters are then used for robust pattern matching.

Recently, an algorithm by Monro and Zhang et al. (University of Bath) [24] claims a near $100 \%$ recognition rate on the CASIA database. They tessellate the iris into rectangular patches oriented at $45^{\circ}$ degrees and then average the patch along its width. The FFT of these 1-D patch signals is taken and the frequency magnitude differences between the adjacent patches and a binary code is generated by detecting the zero crossings of each difference resulting in an iriscode. Hamming distance is used to match two iriscodes.

Park et al. [25] use circular edge detectors for detecting the iris and pupillary boundary. Bandpass filtering is performed on the iris image in order to achieve multiscale characteristics. Each filtered output is subject to a directional filter bank (DFB) which decomposes it into several directional subbands. The subband output is then binarized to form the iris feature vector. The authors also compute directional energy of each subband as a complementary feature vector [26]. The Hamming distance metric is used to match the iris feature vectors.

\subsubsection{Feature-based methods}

Ma et al. [6] propose an algorithm for recognizing irises using key local variations in the iris image. The appearance or disappearance of an important structure in an iris such as crypts, radial fiber, freckle, et. has been used to represent the iris. The spatial locations of these local sharp variation points are computed by first converting the 2-D image into 1-D signal and then applying the dyadic wavelet transform. These position sequences are matched using the exclusive OR operation. Another feature-based iris recognition method has been proposed by Sun et al. [27] who divide the image into small blocks and compute the histograms of the local binary patterns [28] for representing the texture of the iris. Local binary patterns are able to detect corners, edges, line ends, spots, etc. in an image. The similarity between two iris images 
is measured using a global graph matching scheme.

Table 1.1 summarizes the segmentation, encoding (i.e., representation) and matching techniques of some algorithms described in the literature.

\subsection{Challenges in iris recognition}

The significant challenges in iris recognition are enumerated below:

1. The iris acquisition process is inherently difficult. It requires considerable user cooperation and a controlled environment. This may result in acquisition of low quality iris images (occluded, with specular reflections etc.,) and deteriorate the performance of recognition system. Designing techniques to process such poor quality iris images is a challenging task.

2. Iris segmentation is one of the most significant step in iris recognition system. Most of the existing iris segmentation algorithms rely on parametric models of the circle and ellipse to localize the iris. However, when the iris is severely occluded due to the eyelids and the eyelashes, the boundary of the iris may not conform to the circular or elliptical shape.

3. The accuracy of iris recognition is to some extent dependant on the color of the eye. For e.g., when the iris acquisition is using the infra-red spectrum, the brown irises flaunt more textural information as compared to a blue eye.

4. The eye-diseases also have an impact on the recognition performance. For instance, the treatment for glaucoma changes the iris pigmentation and hence the color of the iris [29].

5. Printed contact lenses for eye pose another significant challenge in iris recognition. Some contact lenses have the company logo or some characters printed on them which might conceal the iris information to some extent. Also, iris recognition systems might not be able to detect contact lenses with irides itself printed on them (spoofing).

\subsection{Contributions of this thesis}

The work done in this thesis addresses some of the challenges discussed in the previous section. The major contributions of this thesis are 


\begin{tabular}{|c|c|c|c|}
\hline Authors & $\begin{array}{l}\text { Segmentation } \\
\text { Technique }\end{array}$ & $\begin{array}{l}\text { Encoding } \\
\text { technique }\end{array}$ & $\begin{array}{l}\text { Matching } \\
\text { technique }\end{array}$ \\
\hline Daugman [4] & $\begin{array}{l}\text { Integro-differential } \\
\text { operator }\end{array}$ & $\begin{array}{l}\text { Multiscale 2-D Gabor } \\
\text { Wavelet coefficients }\end{array}$ & Hamming distance \\
\hline Wildes [11] & $\begin{array}{l}\text { Edge detection and } \\
\text { Hough transform }\end{array}$ & $\begin{array}{l}\text { Laplacian-of-a-Gaussian } \\
\text { (LOG) filter }\end{array}$ & Normalized correlation \\
\hline $\begin{array}{l}\text { Bole and } \\
\text { Boashash [14] }\end{array}$ & $\begin{array}{l}\text { Edge and contour } \\
\text { detection }\end{array}$ & $\begin{array}{l}\text { Zero crossings of } \\
\text { Wavelet transform } \\
\text { on the iris }\end{array}$ & Dissimilarity function \\
\hline $\begin{array}{l}\text { Masek [18] } \\
\text { and Kovesi }\end{array}$ & $\begin{array}{l}\text { Kovesi's edge detection } \\
\text { and Hough transform }\end{array}$ & 1D Log-Gabor filters & Hamming distance \\
\hline Ma et al. [12] & $\begin{array}{l}\text { Edge detection and } \\
\text { Hough transform }\end{array}$ & $\begin{array}{l}\text { Circular symmetric } \\
\text { Gabor filters }\end{array}$ & Nearest Feature Line \\
\hline Lim et al. [15] & $\begin{array}{l}\text { Edge detection and } \\
\text { Hough transform }\end{array}$ & $\begin{array}{l}\text { 2-D Harr Wavelet } \\
\text { transform }\end{array}$ & $\begin{array}{l}\text { Competitive learning } \\
\text { neural network }\end{array}$ \\
\hline Noh et al. [8] & $\begin{array}{l}\text { No information } \\
\text { about segmentation }\end{array}$ & ICA & Hamming distance \\
\hline Ma et al. [6] & $\begin{array}{l}\text { Edge detection and } \\
\text { Hough transform }\end{array}$ & $\begin{array}{l}\text { Key local variations } \\
\text { using Wavelet transform }\end{array}$ & Exclusive OR operation \\
\hline Huang et al. [20] & $\begin{array}{l}\text { Edge detection and } \\
\text { Hough transform }\end{array}$ & $\begin{array}{l}\text { Bank of Log-Gabor } \\
\text { filters representing } \\
\text { local orientation of iris }\end{array}$ & Euclidean distance \\
\hline Huang et al. [21] & $\begin{array}{l}\text { Phase congruency and } \\
\text { Hough transform }\end{array}$ & $\begin{array}{l}\text { Multiscale 2-D Gabor } \\
\text { Wavelet coefficients }\end{array}$ & Hamming distance \\
\hline Yuan et al. [22] & $\begin{array}{l}\text { Edge detection and } \\
\text { Hough transform }\end{array}$ & 2-D phase congruency & Euclidean distance \\
\hline Dorairaj et al. [9] & $\begin{array}{l}\text { Integro-differential } \\
\text { operator }\end{array}$ & PCA and ICA & Euclidean distance \\
\hline Thornton et al. [23] & Hough transform & $\begin{array}{l}\text { Advanced correlation } \\
\text { filter }\end{array}$ & Peak-to-correlation-energy \\
\hline $\begin{array}{l}\text { Abhyankar [16] } \\
\text { and Schuckers }\end{array}$ & Active Shape Models & Bi-orthogonal Wavelet & Hamming distance \\
\hline
\end{tabular}

Table 1.1: Examples of a few iris segmentation, encoding and matching techniques proposed in the literature. 
1. A novel iris segmentation scheme that employs Geodesic Active Contours to extract the iris from the surrounding structures has been developed. The proposed scheme elicits the iris texture in an iterative fashion depending upon both the local and global conditions in the image. Also, a post matching algorithm that observes the structure of the differences in feature templates to enhance recognition accuracy has been discussed. The significance of the scheme is its robustness to inaccuracies in the iris segmentation process.

2. An iris recognition system using multiple instances and multiple units of irises has been proposed to offset the disadvantage of encountering poor quality irises. A very good improvement in performance is achieved using multi-instance multi-unit iris recognition.

3. Due to the unavailability of large publicly available iris database, the possibility of generating synthetic iris databases is an attractive alternative. In this thesis, we discuss a new technique for generating synthetic iris images. Here, the background texture is first generated using a texture synthesis scheme based on Markov Random Fields. Then, features of the iris such as the radial and concentric furrows, collarette and crypts are added to the synthetic images.

The organization of the thesis is as follows: Chapter 2 explains a novel iris segmentation scheme using Geodesic Active Contours and illustrates its performance on the CASIA and WVU non-ideal iris databases. It also discusses a technique that studies the block-wise statistics of the feature values constituting the iriscodes to improve the performance of an iris recognition system. The significance of the scheme is its robustness to inaccuracies in the iris segmentation process. Chapter 3 demonstrates the performance improvement achieved in an iris recognition system using multiple instances of a person's iris as well as both units (i.e., both left and right irises) of a person. Chapter 4 discusses a technique to synthesize irises by the means of feature agglomeration. Finally chapter 5 summarizes the contribution of this thesis and suggests extensions to this thesis. 


\section{Chapter 2}

\section{Iris Segmentation using Geodesic}

\section{Active Contours}

\subsection{Motivation}

The first and, perhaps, the most important step in an iris recognition system is iris segmentation or localization. Segmentation involves detecting and isolating the iris structure from an image of the eye. As seen in Figure 1.1, the iris appears to be located in the vicinity of the sclera, the pupil and the eyelids. Thus, the segmentation process has to accurately detect the boundaries separating the iris from these other components. Apart from estimating the actual shape of the iris, the segmentation routine should detect occlusions due to eyelashes that can confound the extracted features. Errors in segmentation can result in inferior recognition performance since the textural content of the iris will be incorrectly encoded.

Most segmentation models in the literature assume that the pupillary, the limbic and the eyelid boundaries are circular or elliptical in shape. Hence, they focus on determining model parameters that best fit these hypotheses ( [4], [11], [6]). Only very few algorithms in the literature do not assume circular or elliptical boundaries (e.g., see Abhyankar and Schuckers [16]). This chapter attempts to address a few primary questions: Can the matching accuracy of an iris recognition system be improved even if the iris is not accurately segmented, and the eyelash and eyelid structures infringe on the segmented iris? Can an alternate iris detection scheme be developed that does not rely on parametric models of the circle and ellipse to localize the iris? Also, should we reject irises which are highly occluded and thus, exhibit very less iris texture 


\section{CHAPTER 2. IRIS SEGMENTATION USING GEODESIC ACTIVE CONTOURS11} pattern?

Thus, the contributions of this chapter are as follows:

1. The first contribution is a novel matching scheme that examines the block-wise structure of differences in iriscodes in order to compare two irises. While most algorithms in the literature merely compute the Hamming distance between two iriscodes, the proposed scheme studies the statistics of the feature values constituting the iriscodes. The significance of the scheme is its robustness to inaccuracies in the iris segmentation process.

2. A novel Geodesic Active Contour-based (GAC) iris segmentation approach that is able to accurately determine the boundary of the iris thereby eliciting its shape. As the iris boundary is not approximated as a circle or an ellipse in the proposed scheme, only the iris pixels are expected to contribute to the localized iris region. The use of Active Contours is not new in the field of image segmentation. They have been extensively used in the field of medical image analysis for segmenting various images like brain MRI images [30], abdominal CT images [31]. They have also been used in the field of machine vision [32]. In this chapter, their significance in the context of efficient iris segmentation is demonstrated.

3. An iris rejection criteria in terms of the amount of iris pattern present in the image has been presented.

\subsection{Baseline iris segmentation, encoding and matching algorithm}

We first describe a baseline segmentation, encoding and matching algorithm that will be used in several of the experiments outlined in this thesis. Integro-differential operators, which are a variation of the Hough transform, act as circular edge detectors and have been formerly used to determine the inner and the outer boundaries of the iris [4]. They also have been used to determine the elliptical boundaries of the lower and the upper eyelids. An integro-differential operator can be defined as

$$
\max _{\left(r, x_{0}, y_{0}\right)}\left|G_{\sigma}(r) * \frac{\partial}{\partial r} \oint_{r, x_{0}, y_{0}} \frac{I(x, y)}{2 \pi r} d s\right|
$$




\section{CHAPTER 2. IRIS SEGMENTATION USING GEODESIC ACTIVE CONTOURS 12}

where $I(x, y)$ is the image, $r$ is the radius of the pupil or iris, $\left(x_{0}, y_{0}\right)$ its center and $G_{\sigma}(r)$ is the Gaussian smoothing function with scale $\sigma$. Thus the integro-differential operator searches for a maximum partial derivative of the image over a circular boundary by varying the radius $r$ and center $\left(x_{0}, y_{0}\right)$. The eyelids can be detected in a similar fashion by performing the integration on an elliptical boundary rather than a circular one. The output of the segmentation process is a binary mask that indicates the iris and non-iris pixels in the image.

Iris segmentation is followed by a normalization scheme to generate a fixed dimension feature vector that lends itself to matching. The rubber sheet model proposed by Daugman maps each point in the $(x, y)$ domain to a pair of polar coordinates $(r, \theta)$. This results in a fixed size unwrapped rectangular iris image [4]. Gabor filters are then used to extract the textural information (encoding) from the unwrapped iris. A 2-D Gabor filter over an image domain $(x, y)$ is given by

$$
G(x, y)=e^{-\pi\left[\left(x-x_{0}\right)^{2} / \alpha^{2}+\left(y-y_{0}\right)^{2} / \beta^{2}\right]} e^{-2 \pi i\left[u_{0}\left(x-x_{0}\right)+\nu_{0}\left(y-y_{0}\right)\right]}
$$

where $\left(x_{0}, y_{0}\right)$ specifies the center of the Gaussian filter, $\alpha$ and $\beta$ are the width and length of the filter, $\left(u_{0}, \nu_{0}\right)$ specify the modulation with frequency $\omega_{0}=\sqrt{u_{0}^{2}+\nu_{0}^{2}}$ and orientation $\theta=\arctan \left(\nu_{0} / u_{0}\right)$. The prominence of the iris texture changes as one moves away from the pupil. Hence, a set of three Gabor filters with different scales and frequency but the same orientation $\left(0^{\circ}\right)$ are applied to different regions of the "normalized" iris as shown in Figure 2.1. The filtering
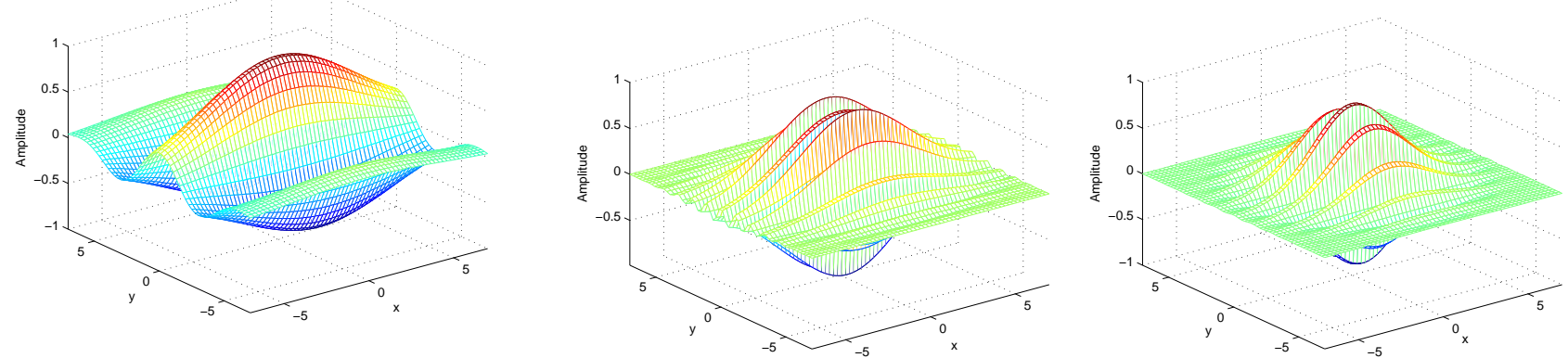

Figure 2.1: Real part of 2-D Gabor wavelet filters.

results in complex-valued phase information. This phase information is quantized into four quadrants in the complex plane resulting in a complex-valued bit whose real and complex parts can be either 0 or 1 . The resulting binary feature vector is called an "iriscode". The difference 
between two such iriscodes is measured using the Hamming distance which is a measure of the number of different bits between the two iriscodes. The Hamming distance is a dissimilarity score and is calculated using the bits corresponding to the iris pixels by utilizing the binary masks generated in the segmentation process. Let $I_{1}$ and $I_{2}$ be the two iriscodes to be compared, and $M_{1}$ and $M_{2}$ be their relative masks. The Hamming distance (HD) is calculated as follows:

$$
H D=\frac{\left\|\left(I_{1} \otimes I_{2}\right) \bigcap M_{1} \bigcap M_{2}\right\|}{\left\|M_{1} \bigcap M_{2}\right\|}
$$

where the XOR operator, $\mathbb{\otimes}$, detects the disagreement between the corresponding bits in the iriscodes, the AND operator, $\bigcap$, ensures that the Hamming distance is calculated using only the bits generated from the true iris region and the $\|$.$\| operator computes the norm of the bit$ vector. Ideally, the Hamming distance between two images of the same iris will be 0 (genuine score) and that between two images of different irises will be (0.5) (impostor score).

\subsection{Enhanced matching utilizing the structure of differ- ences in iriscodes}

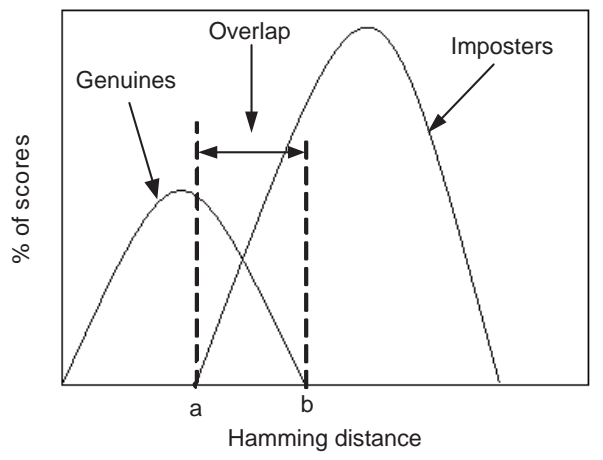

Figure 2.2: The genuine and impostor match score distribution indicating the region of overlap.

Typically, the Hamming distance is computed as a single-valued measure indicating the dissimilarity of two iriscodes. The primary reason for a large Hamming distance for genuine pairs is incorrect iris segmentation or ineffective iris encoding in iris regions having significantly less texture information. Integro-differential operators are not able to segment the highly occluded irises of the CASIA database (Figure 2.3). When an occluded iris image due to eyelids or eyelashes is not segmented accurately, the normalized iris contains non-iris parts of the eye like the sclera 


\section{CHAPTER 2. IRIS SEGMENTATION USING GEODESIC ACTIVE CONTOURS14}

and the eyelids which should have been masked out. Matching the iriscodes (feature templates) of such images results in a large Hamming distance and hence poor iris recognition performance.

The structure of the differences between iriscodes is not exploited when computing the Hamming distance. In this section, it has been demonstrated that the structure of differences in iriscodes can be used to enhance the matching performance. Consider a sample genuine-impostor distribution as shown in Figure 2.2. The ambiguous region, $R=[a, b]$, represents the overlapping of genuine and impostor scores. Also, When a match score falls in the ambiguous region $R$, then the following sequence of steps is executed: (a) The two registered rectangular iriscodes are divided into small blocks; (b) The Hamming distance between corresponding blocks is computed; (c) If more than half the number of blocks have a Hamming distance lower than $a$, then the match score is labeled as a genuine score. As any bit in iriscode can be equally likely either 0 or 1, the probability of two blocks of iriscodes for two different irises matching is 0.5. Daugman [33] has shown that the Hamming distance distribution for impostors lies in the range of 0.45 and 0.55. The Hamming distance for a particular block can also lie in the range of 0.55 and 1 when a significant region of iris is occluded and hence only a few bits in that block of iriscode are to be matched to compute the match score. For e.g., consider two blocks of iriscodes where only 1 bit is unmasked and all others are masked due to occlusion. Also, if that bit in first block of iriscode is 1 and in the other block is 0 , then the Hamming distance between such blocks of iriscodes is 1 . Thus very high Hamming distances might occur due to the masked iris blocks which might increase the overall Hamming distance of two iriscodes. Let $c$ be the maximum theoretical Hamming distance between two iriscodes assuming that they are unmasked. If less than $33 \%$ of the blocks are in $[a, c]$ (which is the HD range for impostors), then the match score is labeled as a genuine score; else, the original Hamming distance is retained. The assumption behind this approach is that, in appropriately registered iris images of the same eye, a high Hamming distance is primarily due to incorrect segmentation. Thus, only a few blocks contribute to large Hamming distances. In the case of an impostor pair, on the other hand, almost all the blocks contribute to a large Hamming distance. The block diagram in Figure 2.4 summarizes this approach.

The performance of this matching algorithm was evaluated on the CASIA iris database after employing integro-differential operators to segment the iris. This database has 756 iris images corresponding to 108 different irises ( 7 images per iris: $I_{1}, I_{2}, \ldots I_{7}$ ). Each image is of size 


\section{CHAPTER 2. IRIS SEGMENTATION USING GEODESIC ACTIVE CONTOURS15}
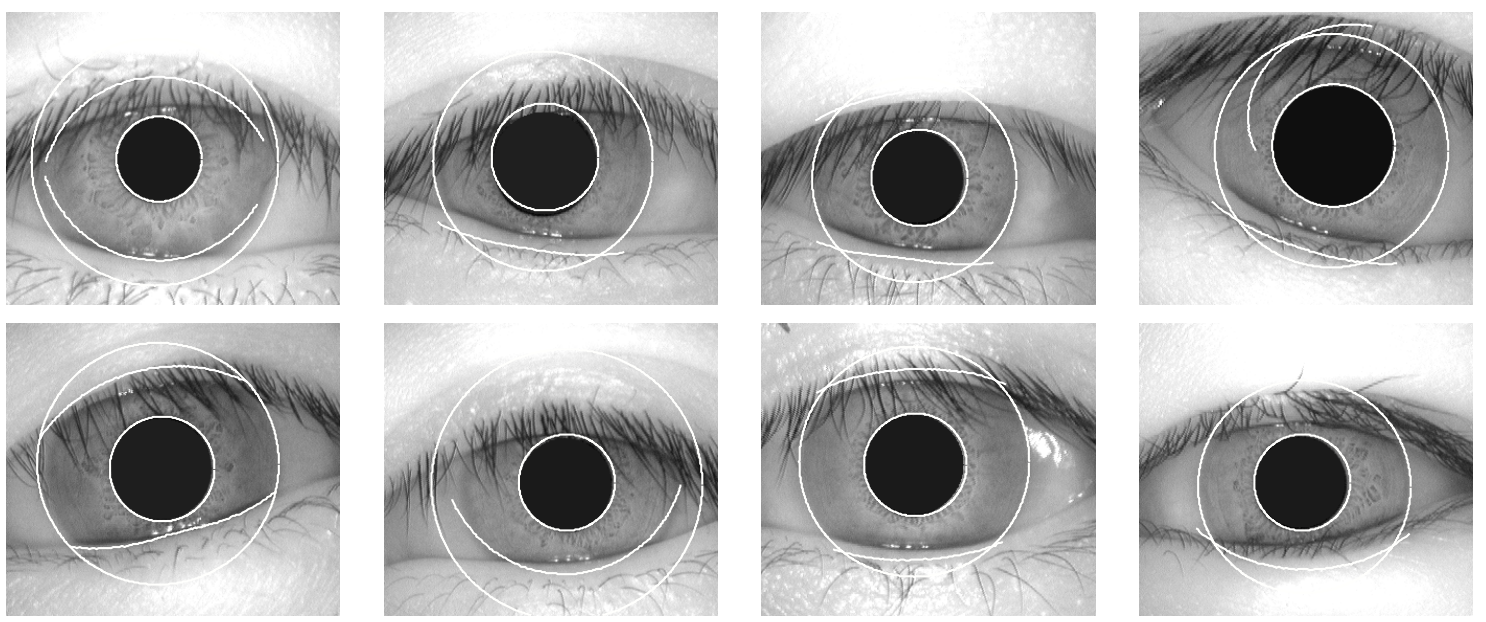

Figure 2.3: Irises incorrectly segmented using the integro-differential operators.

\begin{tabular}{|l|l|l|l|}
\hline FAR (\%) & \multicolumn{3}{|c|}{ GAR (\%) } \\
\hline & Masek's code & $\begin{array}{l}\text { integro-differential operators } \\
\text { for segmentation }\end{array}$ & $\begin{array}{l}\text { Statistical analysis of } \\
\text { iriscodes }\end{array}$ \\
\hline 1 & 94.0 & 94.2 & 97.4 \\
\hline 0.1 & 92.6 & 93.8 & 96.4 \\
\hline 0.01 & 88.0 & 91.5 & 95.2 \\
\hline 0.001 & 82.0 & 90.0 & 92.0 \\
\hline
\end{tabular}

Table 2.1: The GAR at a fixed FAR using Masek's code, integro-differential operators for iris segmentation and statistical analysis of iriscodes.

$320 \times 280$. Figure 2.5 shows the significant improvement in the performance of the iris recognition system using the proposed technique (in this experiment, $a=0.36, b=0.48$ and $c=0.55$ ). For example, the GAR (Genuine Acceptance Rate), at a fixed FAR (False Acceptance Rate) of $0.01 \%$ using the proposed technique is $95 \%$ while that using Masek's [34] code is $88 \%$ and using our implementation of Daugman's code is 91.5\%. Maseks algorithm [34] refers to the publicly available code developed by Libor Masek for iris segmentation, feature extraction and matching. Table 2.1 summarizes the performance improvement by modifying the structure of differences in iriscodes. 


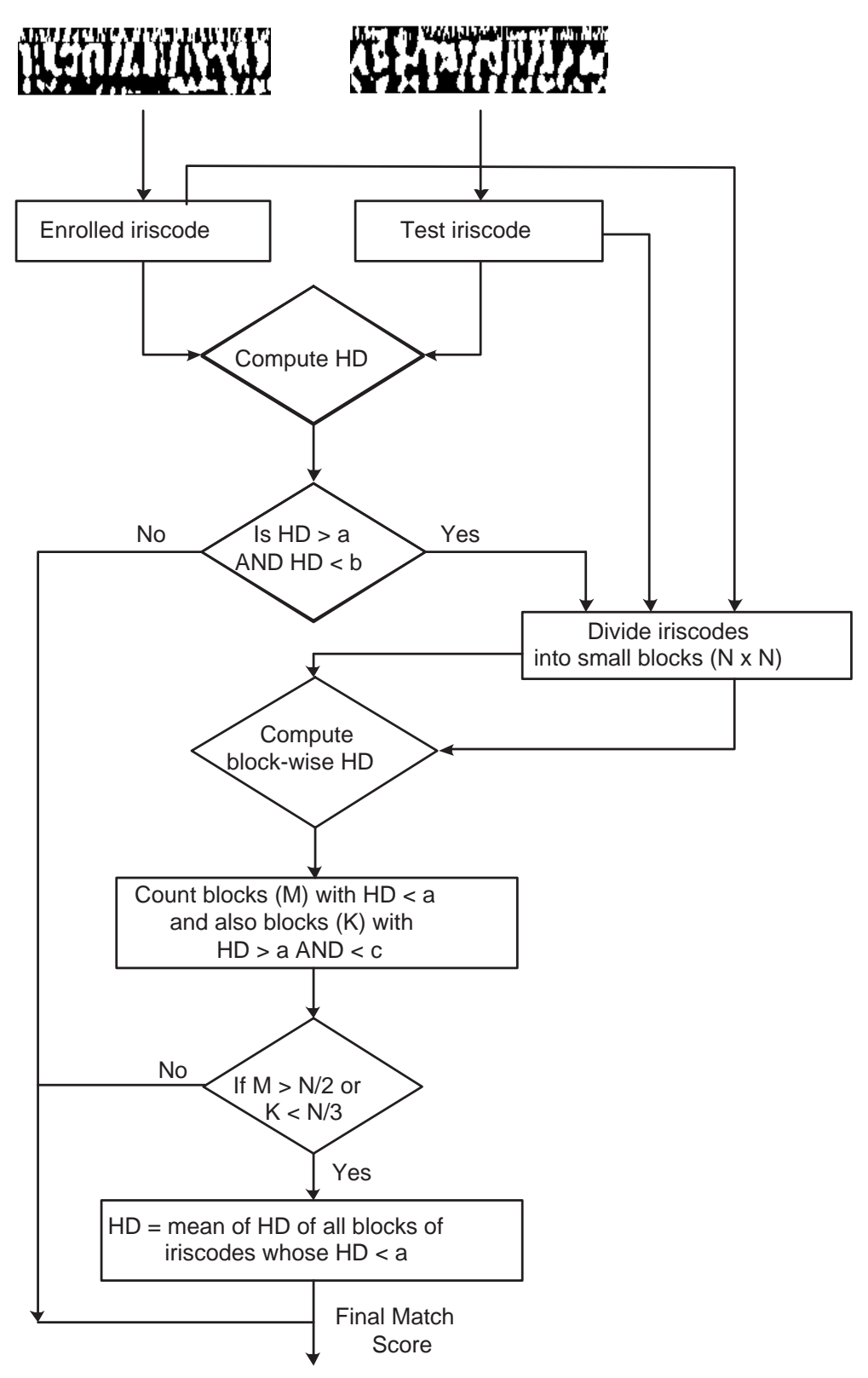

Figure 2.4: The block diagram summarizing the new matching scheme utilizing the block-wise differences in iriscodes. Here $a$ and $b$ are the boundaries of the overlap region between genuine and impostor scores. 


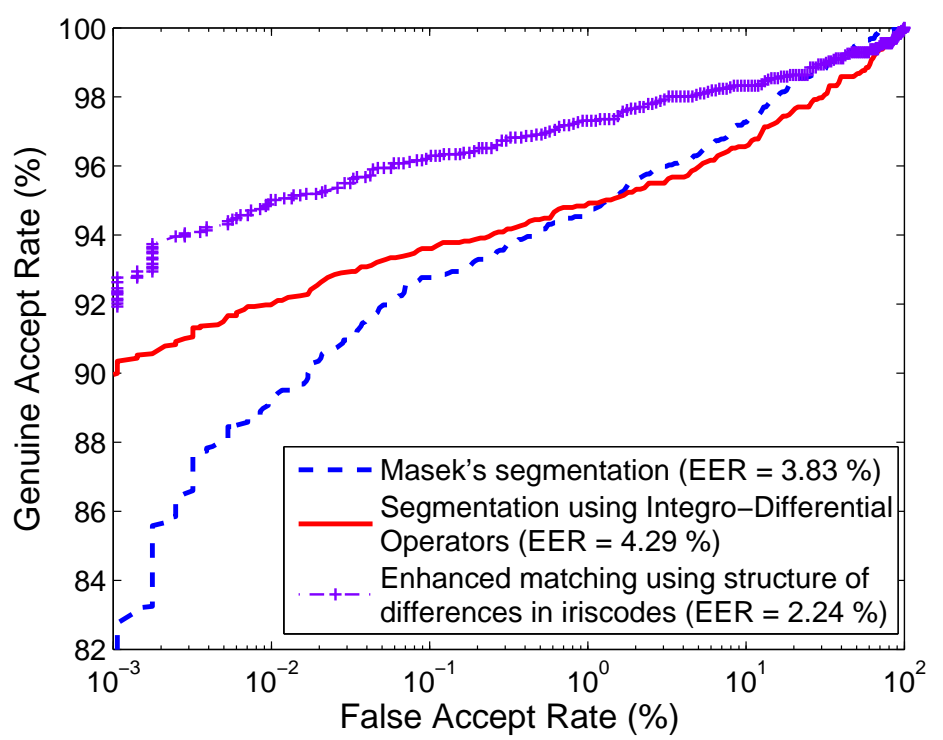

Figure 2.5: ROC curves indicating the performance improvement on the CASIA database using the structure of differences in iriscodes.

\subsection{Iris Segmentation using Geodesic Active Contours}

The iris localization procedure can be divided broadly into two stages: (a) Pupil segmentation and (b) Iris segmentation.

\subsubsection{Pupil Segmentation}

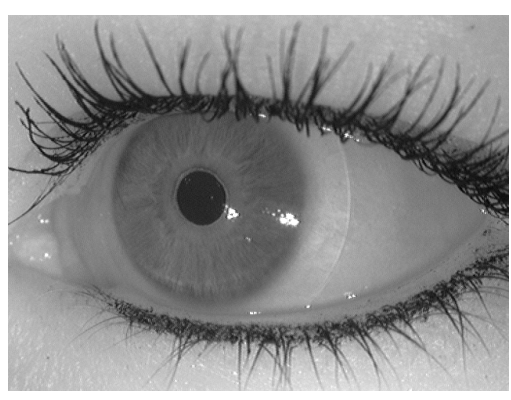

(a)

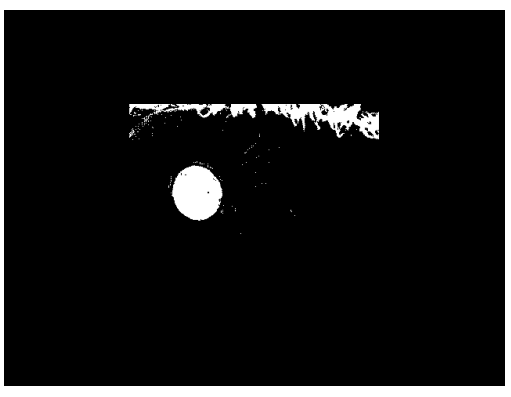

(b)

Figure 2.6: Pupil binarization. (a) Image of an eye with dark eyelashes; (b) Thresholded binary iris image.

To detect the pupillary boundary, the iris image is smoothed using a 2-D median filter and the minimum pixel value $(M)$ is determined. The iris is then binarized using a threshold value $25+M$. Figure 2.6 (b) shows an iris image after binarization. As expected, apart from the pupil, 


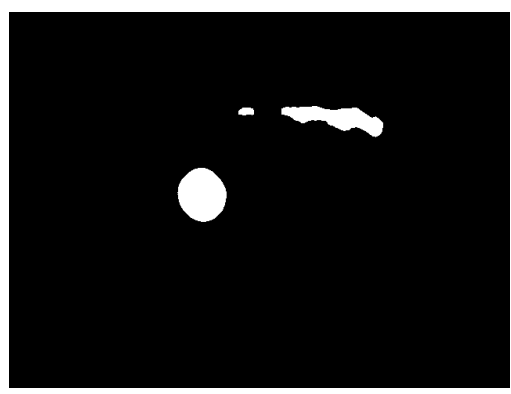

Figure 2.7: 2-D Median filtered binary iris image.

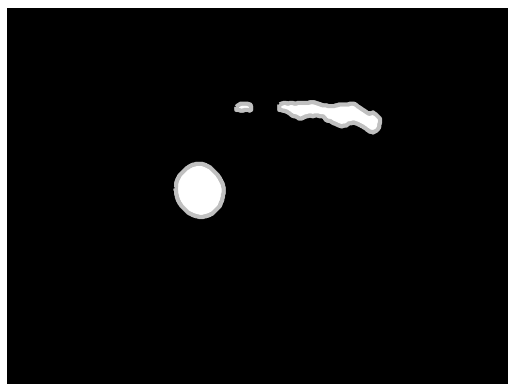

Figure 2.8: Traced boundaries of all the remaining objects in the binary image (shown in gray).

other dark regions of the eye (e.g., eyelashes) fall below this threshold value. A 2-D median filter is then applied on the binary image to discard the relatively smaller regions associated with the eyelashes. This reduces the number of candidate regions detected as a consequence of thresholding as shown in Figure 2.7 (a). Based on the median-filtered binary image, the exterior boundaries of all the remaining objects are traced as shown in Figure 2.8. Generally, the largest boundary of the remaining regions of the eye corresponds to the pupil. However, when the pupil is constricted, it is very likely that the boundary of the detected region corresponding to the eyelashes is larger than that of the pupil. So a circle-fitting procedure is executed on all detected regions. The equation of a circle is given by

$$
x^{2}+y^{2}+a_{1} x+a_{2} y+a_{3}=0
$$

where $(x, y)$ represent the coordinates of a point on the circle. The coordinates of the center can be computed as $x_{0}=-a_{1} / 2, y_{0}=-a_{2} / 2$ and the radius as $R_{i}=\sqrt{\left(a_{1}^{2}+a_{2}^{2}\right) / 4-a_{3}}$. Let $\left\{\left(x_{i}, y_{i}\right) \mid i=1,2, \ldots n\right\}$ be $n$ points traversed by the circle, and let 
CHAPTER 2. IRIS SEGMENTATION USING GEODESIC ACTIVE CONTOURS19

$$
\mathrm{M}=\left[\begin{array}{c}
x_{1}^{2}+y_{1}^{2} \\
x_{2}^{2}+y_{2}^{2} \\
\ldots \\
x_{n}^{2}+y_{n}^{2}
\end{array}\right]_{n \times 1}, \quad \mathrm{~N}=\left[\begin{array}{ccc}
x_{1} & y_{1} & 1 \\
x_{2} & y_{2} & 1 \\
\ldots & \ldots & \ldots \\
x_{n} & y_{n} & 1
\end{array}\right]_{n \times 3} \quad, \quad \mathrm{~A}=\left[\begin{array}{c}
a_{1} \\
a_{2} \\
a_{3}
\end{array}\right]_{3 \times 1}
$$

Then the equation of the circle can be written as $N A=M$ Thus, to find the radius and the center of the circle, the equation $A=N^{-1} M$ needs to be solved. Here, as $N$ is not a square matrix, its inverse is computed using the least squares criterion [35]. Figure 2.9] demonstrates the circles fitted through all the detected regions.

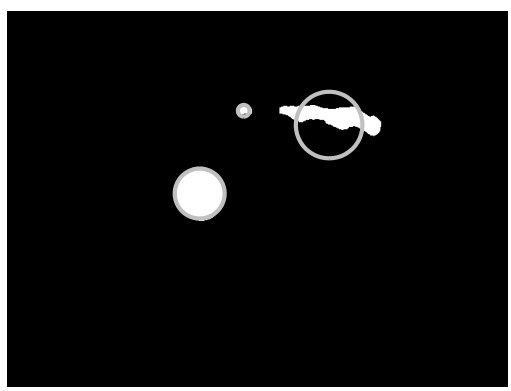

Figure 2.9: Fitting circle on all potential regions where pupil might be present (shown in gray).

Finally, the circle whose circumference contains the maximum number of black pixels is deemed to be the detected pupil. Regions with diameters more than half the image size are not considered. Figure 2.10 shows an iris image containing dark eyelashes and the correctly segmented pupil using the aforementioned algorithm. Sometimes, specular reflections might occur near the boundary of the pupil and hence the pupil segmentation procedure might get affected. For e.g., in the Figure 2.11, it is obvious that the pupil is under-segmented due to the presence of the specular reflection near the pupil boundary. Hence, if specular reflection (bright spots in a image) is detected in close vicinity of the pupil, it is "inpainted" using the surrounding information [36]. Inpainting is a process to fill in the missing portions of am image (in our case specular reflections) to make it look more legible [37]. Figure 2.12 (f) proves that the pupil detection procedure becomes more robust by inpainting the specular reflections in the iris image whenever these reflections occur near the pupil boundary.

Figure 2.13 shows more examples of the pupil segmentation process. 


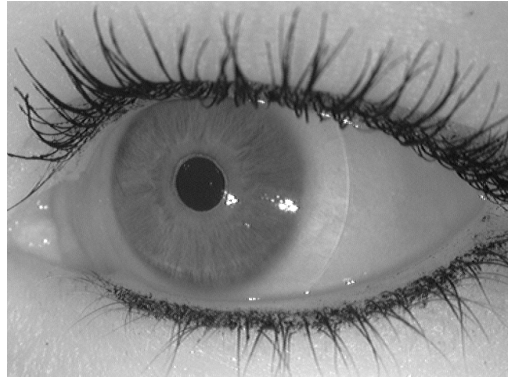

(a)

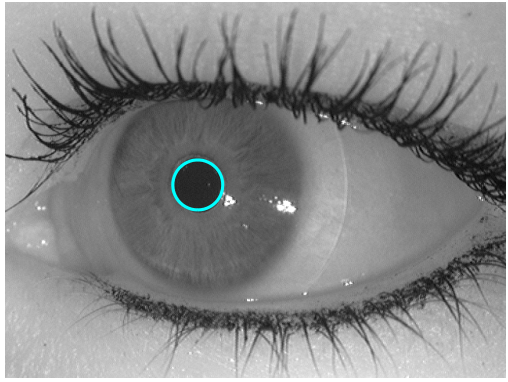

(b)

Figure 2.10: Pupil segmentation. (a) Image of an eye with dark eyelashes; (b) Segmented pupil (shown using white color).

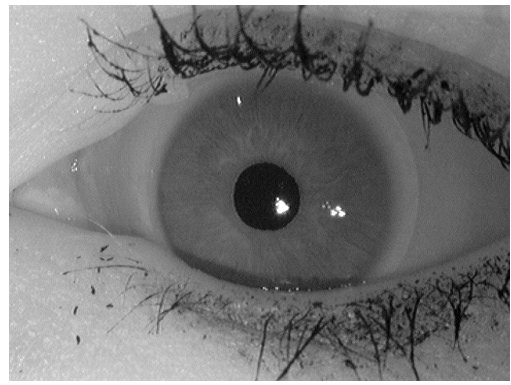

(a)

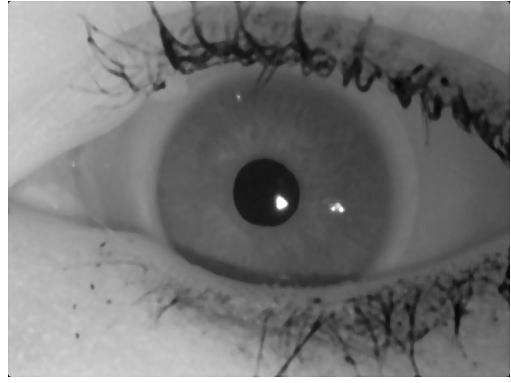

(b)

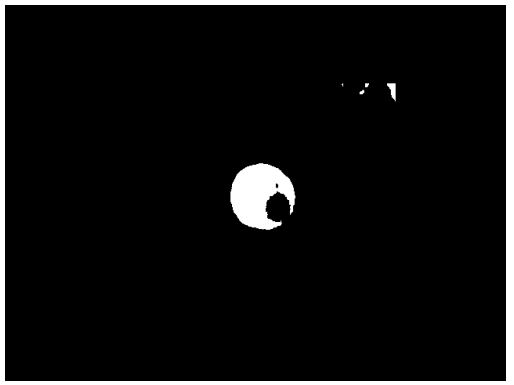

(c)

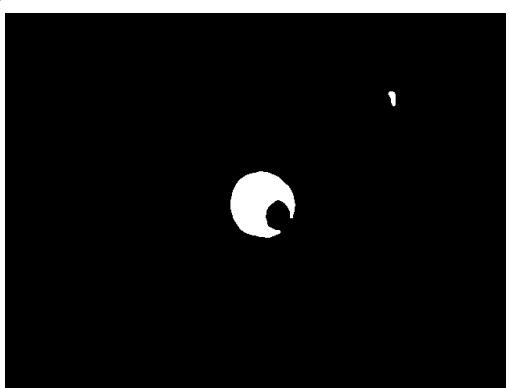

(d)

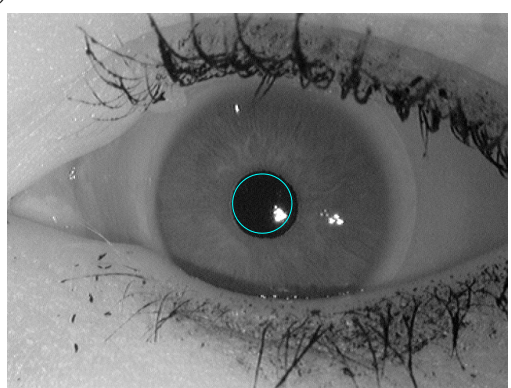

(e)

Figure 2.11: Pupil segmentation without inpainting of specular reflections. (a) Iris image; (b) Smoothed image; (c) Thresholded binary image; (d) 2-D Median filtered image; (e) Undersegmented pupil (shown using white color). 


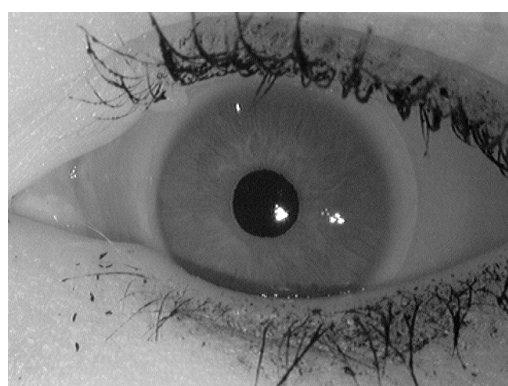

(a)

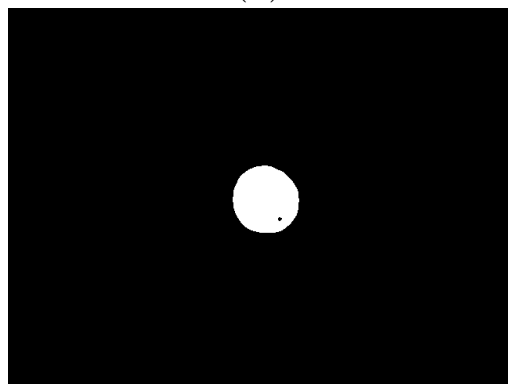

(d)

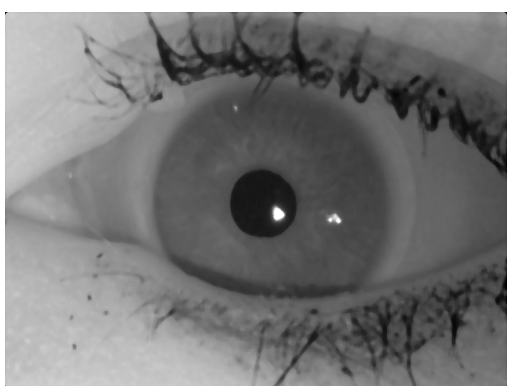

(b)

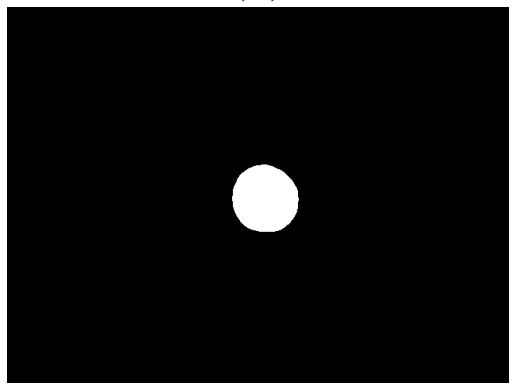

(e)

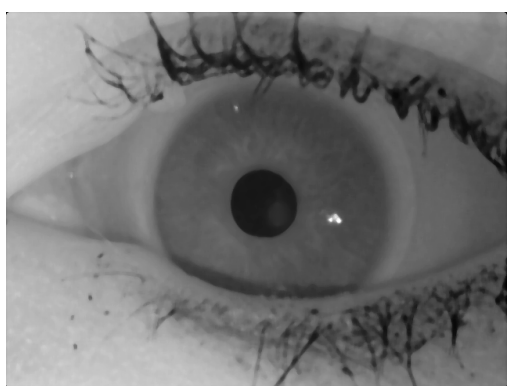

(c)

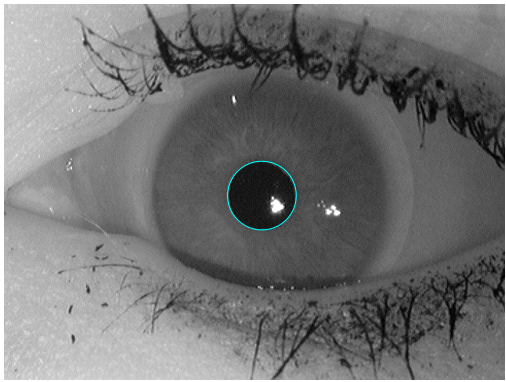

(f)

Figure 2.12: Pupil segmentation using inpainting of specular reflections in the vicinity of the pupil. (a) Iris image; (b) Smoothed image; (c) Inpainted specular reflection in the vicinity of the pupil; (d) Thresholded binary image; (e) 2-D Median filtered image; (f) Segmented pupil (shown using white color).

\subsubsection{Iris segmentation}

To detect the limbic boundary of the iris, a novel scheme based on a level sets representation ( [38], 39], [40], [32]) of geodesic active contour model is employed. This approach is based on the relation between active contours and the computation of geodesics (minimal length curves) [41]. The technique is to evolve the contour from inside the iris under the influence of geometric constraints of the iris image. Geodesic active contours combine the energy minimization approach of classical "snakes" and the geometric active contours based on curve evolution.

\section{Geodesic Active Contours}

Let $\gamma(t)$ be the curve to be evolved towards the boundary of any object at a particular time $t$ as shown in Figure 2.14, Let $\psi$ be a function defined as a signed distance function from the 

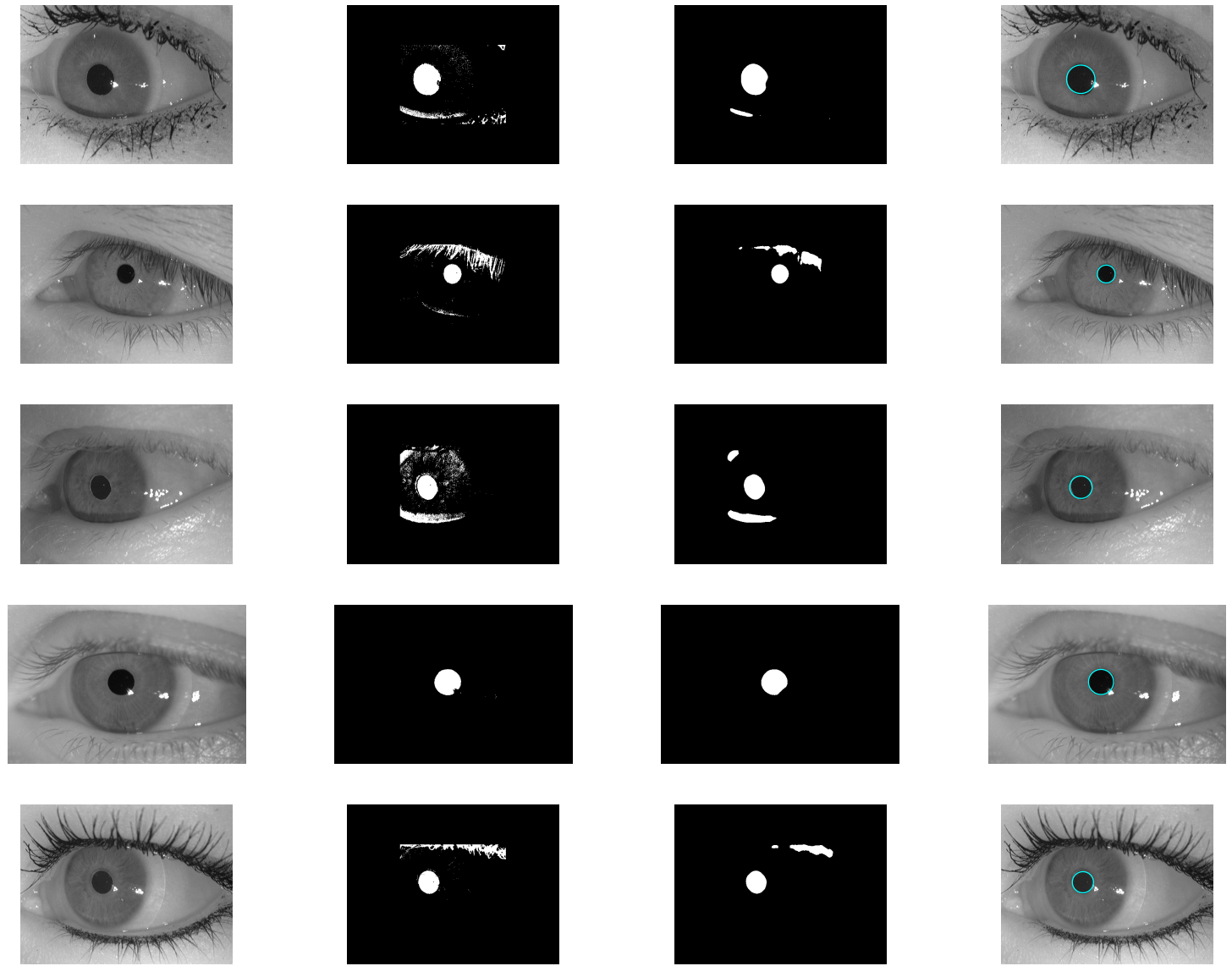

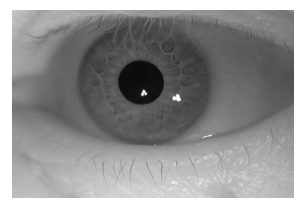

(a)

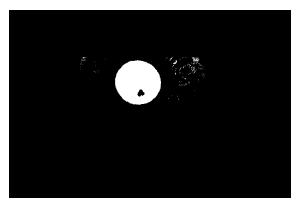

(b)

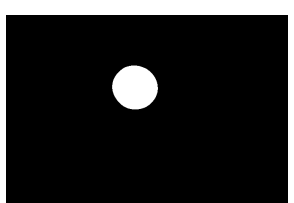

(c)

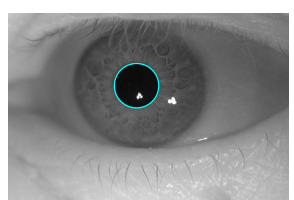

(d)

Figure 2.13: Pupil segmentation. (a) Iris image; (b) Thresholded binary image; (c) 2-D Median filtered image; (d) Segmented pupil (shown using white color). 


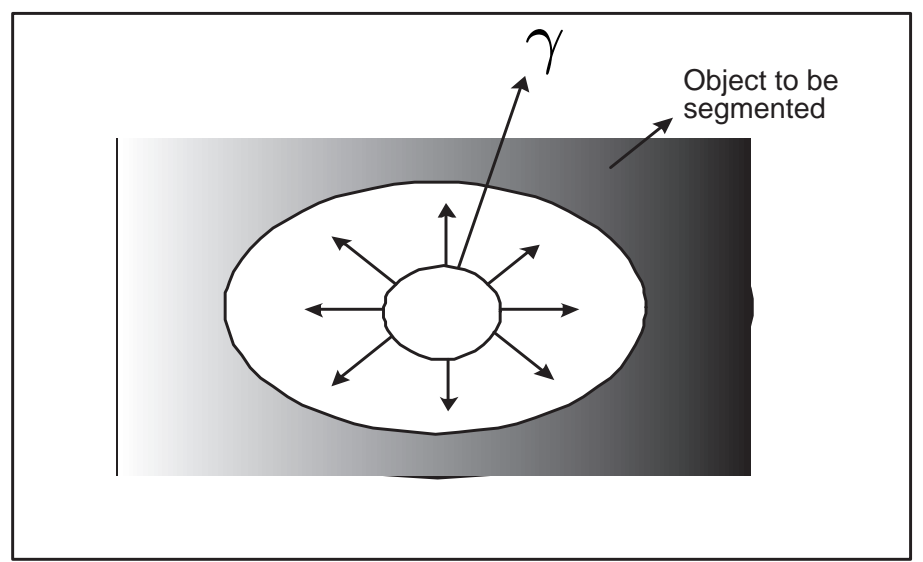

Figure 2.14: Curve $\gamma$ evolving towards the boundary of the object.

curve $\gamma(t)$ [42]. Thus, $\psi(x, y)=$ distance of point $(x, y)$ to the curve $\gamma(t)$.

$$
\psi(x, y)= \begin{cases}0 & \text { if }(\mathrm{x}, \mathrm{y}) \text { is on the curve } \\ <0 & \text { if }(\mathrm{x}, \mathrm{y}) \text { is inside the curve } \\ >0 & \text { if }(\mathrm{x}, \mathrm{y}) \text { is outside the curve }\end{cases}
$$

$\psi$ is of the same dimension as that of the image $I(x, y)$ that is to be segmented. The curve $\gamma(t)$ is a level set of the function $\psi$. Level sets are the set of all points in $\psi$ where $\psi=$ some constant. Thus $\psi=0$ is the zeroth level set, $\psi=1$ is the first level set and so on. $\psi$ is the implicit representation of the curve $\gamma(t)$ and is called as the embedding function since it embeds the evolution of $\gamma(t)$. The embedding function evolves under the influence of image gradients and regions characteristics so that the curve $\gamma(t)$ approaches the boundary of the object. Thus, instead of evolving the parametric curve $\gamma(t)$ (e.g., the Lagrangian approach used in snakes), the embedding function itself is evolved. In our algorithm, the initial curve $\gamma(t)$ is assumed to be a circle of radius $r$ just beyond the pupillary boundary. Let the curve $\gamma(t)$ be the zeroth-level set of the embedding function. This implies that

$$
\frac{d \psi}{d t}=0 .
$$

By the chain rule,

$$
\frac{d \psi}{d t}=\frac{\partial \psi}{\partial x} \frac{d x}{d t}+\frac{\partial \psi}{\partial y} \frac{d y}{d t}+\frac{\partial \psi}{\partial t}
$$

i.e.

$$
\frac{\partial \psi}{\partial t}=-\nabla \psi \cdot \gamma^{\prime}(t)
$$


Splitting the $\gamma^{\prime}(t)$ in the normal $(N(t))$ and tangential $(T(t))$ directions,

$$
\frac{\partial \psi}{\partial t}=-\nabla \psi \cdot\left(v_{N} N(t)+v_{T} T(t)\right)
$$

Now, since $\nabla \psi$ is perpendicular to the tangent to $\gamma(t)$,

$$
\frac{\partial \psi}{\partial t}=-\nabla \psi \cdot\left(v_{N} N(t)\right)
$$

The normal component is given by

$$
N=\frac{\nabla \psi}{\|\nabla \psi\|}
$$

Substituting this in equation 2.4 ,

$$
\frac{\partial \psi}{\partial t}=-v_{N}\|\nabla \psi\|
$$

Let $v_{N}$ be a function of the curvature of the curve $\kappa$, stopping function $K$ (to stop the evolution of the curve) and the inflation force $c$ (to evolve the curve in the outward direction) such that,

$$
\frac{\partial \psi}{\partial t}=-\left(\operatorname{div}\left(K \frac{\nabla \psi}{\|\nabla \psi\|}\right)+c K\right)\|\nabla \psi\|
$$

Thus, the evolution equation for $\psi_{t}$ such that $\gamma(t)$ remains the zero level set is given by

$$
\psi_{t}=-K(c+\epsilon \kappa)\|\nabla \psi\|+\nabla \psi \cdot \nabla K
$$

where, $K$, the stopping term for the evolution, is an image dependant force and is used to decelerate the evolution near the boundaries; $c$ is the velocity of the evolution; $\epsilon$ indicates the degree of smoothness of the level sets; and $\kappa$ is the curvature of the level sets computed as

$$
\kappa=-\frac{\psi_{x x} \psi_{y}^{2}-2 \psi_{x} \psi_{y} \psi_{x y}+\psi_{y y} \psi_{x}^{2}}{\left(\psi_{x}^{2}+\psi_{y}^{2}\right)^{\frac{3}{2}}}
$$

where $\psi_{x}$ is the gradient of the image in the $x$ direction; $\psi_{y}$ is the gradient in the $y$ direction; $\psi_{x x}$ is the $2^{\text {nd }}$ order gradient in the $x$ direction; $\psi_{y y}$ is the $2^{\text {nd }}$ order gradient in the $y$ direction; and $\psi_{x y}$ is the $2^{n d}$ order gradient, first in the $x$ direction and then in the $y$ direction. Equation 2.5 is the level set representation of the geodesic active contour model. This means that the level-set $C$ of $\psi$ is evolving according to

$$
C_{t}=K(c+\epsilon \kappa) \vec{N}-(\nabla K \cdot \vec{N}) \vec{N}
$$

where $\vec{N}$ is the normal to the curve. The first term $(\kappa \vec{N})$ provides the smoothing constraints on the level sets by reducing the total curvature of the level sets. The second term $(c \vec{N})$ acts 


\section{CHAPTER 2. IRIS SEGMENTATION USING GEODESIC ACTIVE CONTOURS25}

like a balloon force [43] and it pushes the curve outward towards the object boundary. The goal of the stopping function is to slow down the evolution when is reaches the boundaries. However, the evolution of the curve will terminate only when $K=0$, i.e., near an ideal edge. In most images, the gradient values will be different along the edge, thus necessitating different $\mathrm{K}$ values. In order to circumvent this issue, the third geodesic term $((\nabla K . \vec{N}))$ is necessary so that the curve is attracted toward the boundaries ( $\nabla K$ points towards the middle of the boundary). This term makes it possible to terminate the evolution process even if (a) the stopping function has different values along the edges, and (b) gaps are present in the stopping function.

The stopping term used for the evolution of level sets is given by

$$
K(x, y)=\frac{1}{1+\left(\frac{\|\nabla(G(x, y) \star I(x, y))\|}{k}\right)^{\alpha}}
$$

where $I(x, y)$ is the image to be segmented, and $k$ and $\alpha$ are constants. As can be seen, this term $K(x, y)$ is not a function of $t$.

\section{Iris segmentation using Geodesic Active Contours}

Consider an iris image (CASIA) to be segmented as shown in Figure 2.15 (a). The stopping function, $K$, obtained from this image is shown in Figure 2.15 (b) (In our implementation, for CASIA images, $k=1.6$ and $\alpha=10$ and for WVU non-ideal images, $k=2.8$ and $\alpha=8$ ). As the pupil segmentation is done prior to segmenting the iris, the stopping function $K$ is modified by deleting the circular edges because of the pupillary boundary, resulting in a new stopping function $K^{\prime}$. This ensures that the evolving level set is not terminated by the edges of the pupillary boundary (Figure 2.15 (c)).

A contour is first initialized near the pupil (Figure 2.16 (a)). The embedding function $\psi$ is initialized as a signed distance function to $\gamma(t=0)$ which looks like a cone (Figure 2.16 (b)). Discretizing equation 2.5 leads to the following equation:

$$
\frac{\psi_{i, j}^{t+1}-\psi_{i, j}^{t}}{\Delta t}=-c K_{i, j}^{\prime}\left\|\nabla \psi^{t}\right\|-K_{i, j}^{\prime}\left(\epsilon \kappa_{i, j}^{t}\left\|\nabla \psi^{t}\right\|\right)+\nabla \psi_{i, j}^{t} . \nabla K_{i, j}^{\prime t}
$$

where $\Delta t$ is the time step. In our implementation, $\Delta t$ is 0.05 . The first term $\left(c K_{i, j}^{\prime}\left\|\nabla \psi^{t}\right\|\right)$ on the right hand side of the above equation is the velocity term (advection term) and in the case of iris segmentation, acts as an inflation force. This term can lead to singularities and hence is discretized using upwind finite differences [44]. The upwind scheme for approximating $\|\nabla \psi\|$ is 


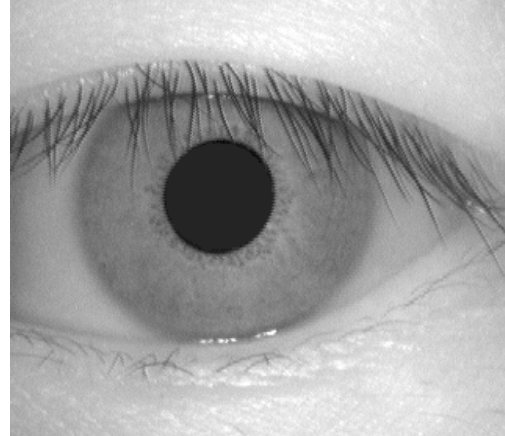

(a)

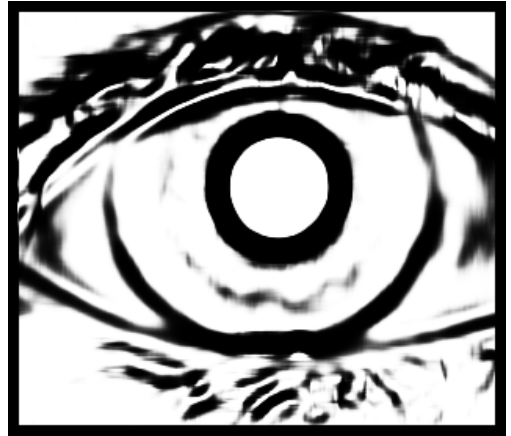

(b)

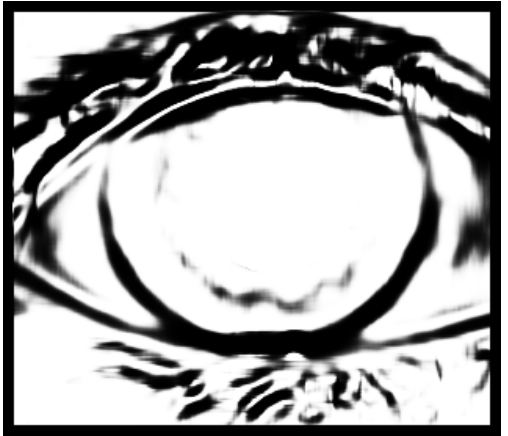

(c)

Figure 2.15: Stopping function for the geodesic active contours.(a) Example iris image from CASIA user 5; (b) Stopping function $K$; (c) Modified stopping function $K^{\prime}$.

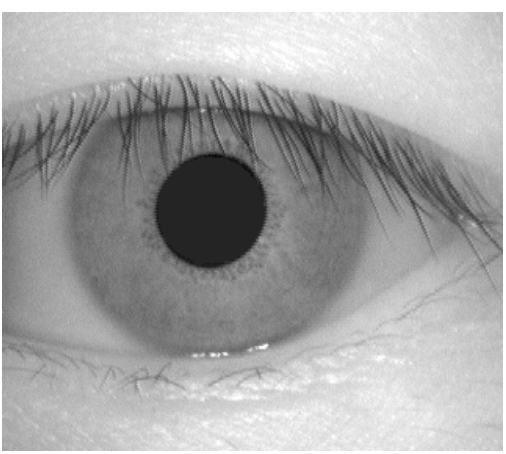

(a)

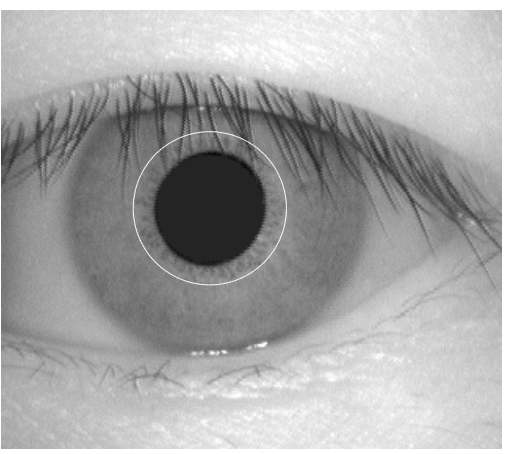

(b)

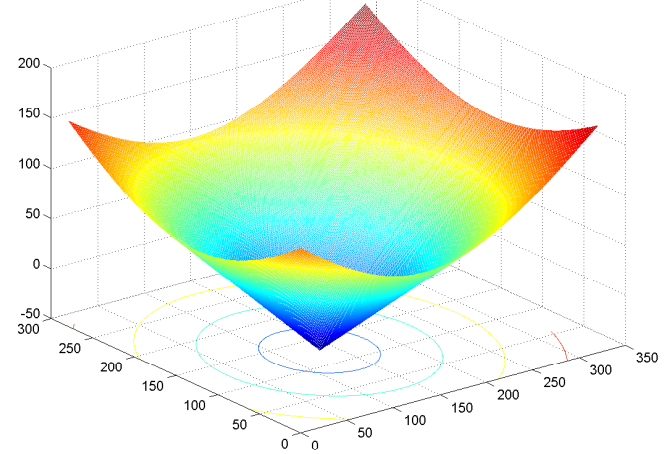

(c)

Figure 2.16: Contour initialization for iris segmentation.(a) Example iris image from CASIA user 5; (b) Zero level set (initial contour); (c) Mesh plot of the signed distance function $\psi$. 


\section{CHAPTER 2. IRIS SEGMENTATION USING GEODESIC ACTIVE CONTOURS 27}

given by

$$
\|\nabla \psi\|=\sqrt{\left.\left.\left.\left.\min \left(D_{x}^{-} \psi_{i, j}, 0\right)^{2}\right)+\max \left(D_{x}^{+} \psi_{i, j}, 0\right)^{2}\right)+\min \left(D_{y}^{-} \psi_{i, j}, 0\right)^{2}\right)+\min \left(D_{y}^{+} \psi_{i, j}, 0\right)^{2}\right)},
$$

where

$D_{x}^{-} \psi$ is the first order backward difference of $\psi$ in the x-direction; $D_{x}^{+} \psi$ is the first order forward difference of $\psi$ in the x-direction; $D_{y}^{-} \psi$ is the first order backward difference of $\psi$ in the y-direction; and $D_{y}^{+} \psi$ is the first order forward difference of $\psi$ in the y-direction. The second term $\left(K_{i, j}^{\prime}\left(\epsilon \kappa_{i, j}^{t}\left\|\nabla \psi^{t}\right\|\right)\right)$ is a curvature based smoothing term and can be discretized using central differences. In our implementation, $c=0.65$ and $\epsilon=1$ for all iris images. The third geodesic term $\left(\nabla \psi_{i, j}^{t} . \nabla K_{i, j}^{\prime t}\right)$ is also discretized using the central differences.

After evolving the embedding function $\psi$ according to equation 2.8, the curve starts to grow until it satisfies the stopping criterion defined by the stopping function $K^{\prime}$. The difference between the zeroth level sets after every 50 iterations is calculated. If this difference is less than a threshold, then the evolution is stopped. This criterion minimizes the thin plate spline energy [45] (square of the curvature) of the level sets. It stops the level sets from evolving only in a particular region of the iris. In our implementation, this threshold is set to 1 . The evolution of the curve and the corresponding embedding functions are illustrated in Figure 2.17.

If the Thin Plate Spline energy of the level sets is not minimized, the contour might continue to evolve if the stopping function does not have a high magnitude. Thus, the contour will encompass some portion of the sclera inside the final contour as shown in Figure 2.18 (a). Minimizing the thin plate spline energy of level sets yields the exact contour of the iris (Figure $2.18(\mathrm{~b})$ ).

One important feature of geodesic active contours is their ability to handle splitting and merging boundaries. This phenomenon is especially important in the case of iris segmentation since the radial fibers may be very thick in some parts of the iris, or the crypts present in the ciliary region may be very dark, leading to prominent edges in the stopping function. If the segmentation technique is based on parametric curves (e.g., the snakes segmentation technique [46], then the evolution of the curve might terminate at these local minima. However, geodesic active contours are able to split at such local minima and merge again. Thus, they are able to effectively deal with the problems of local minima and hence the final contour corresponds to the true iris boundary (Figure 2.19).

Since, during the evolution process, we are primarily interested only in the zeroth level set 


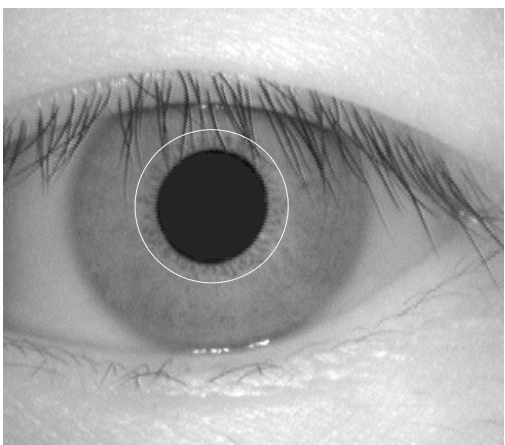

(a)

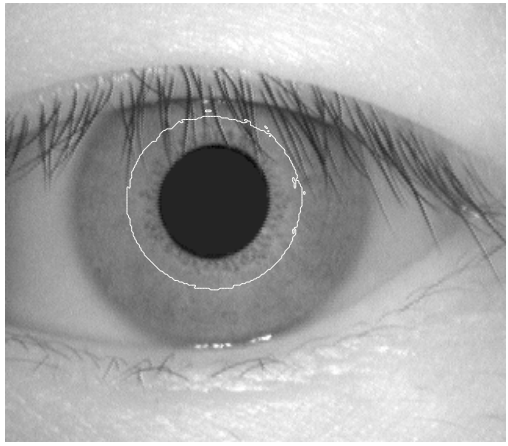

(c)

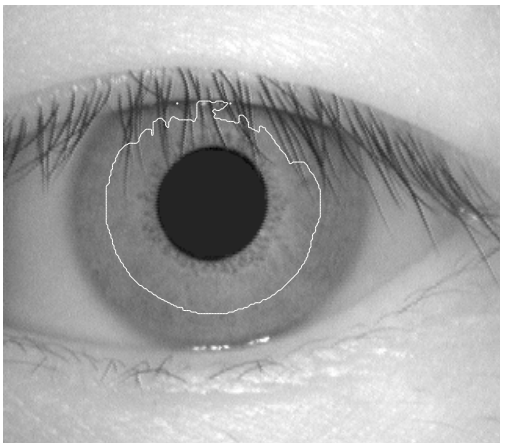

(e)

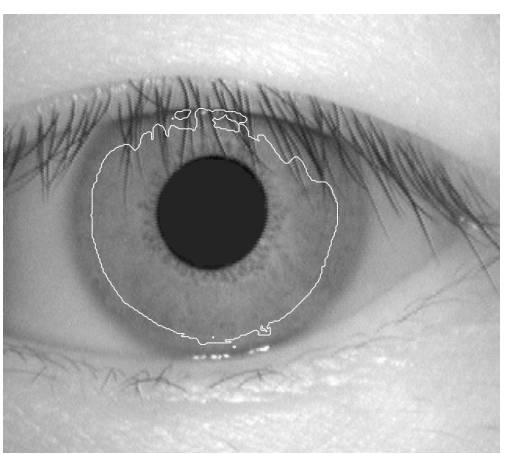

(g)

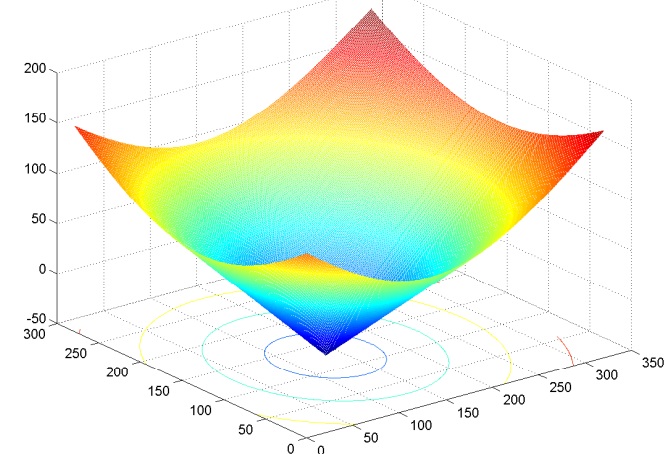

(b)

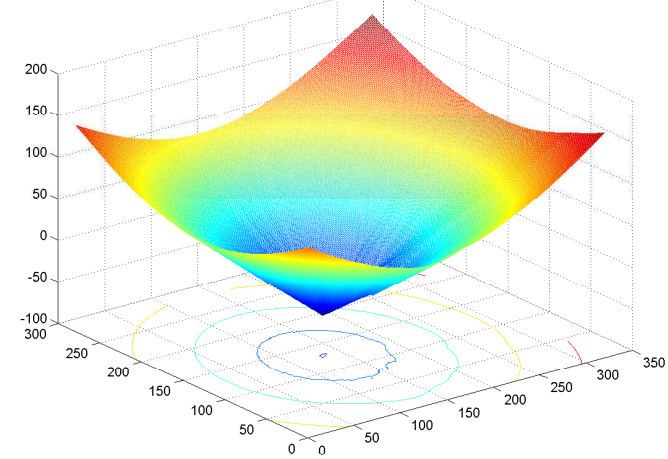

(d)

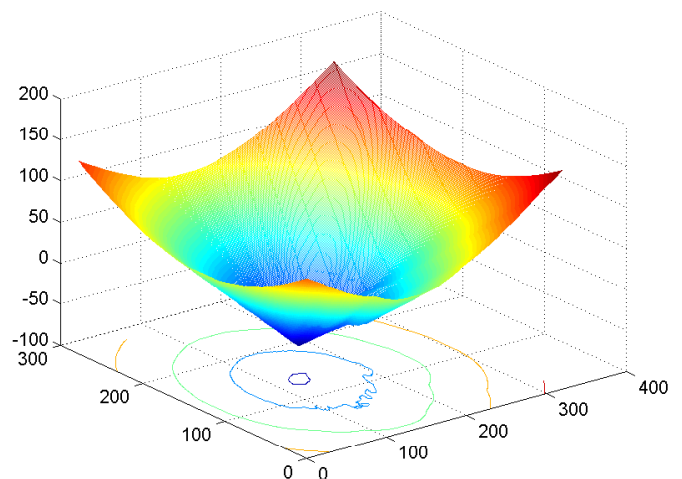

(f)

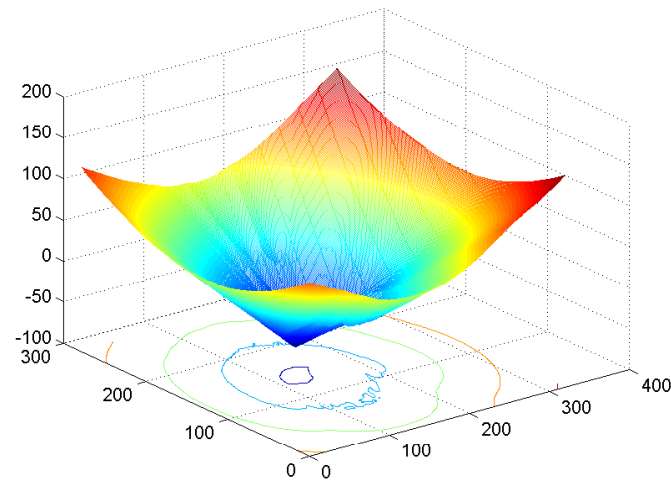

(h) 


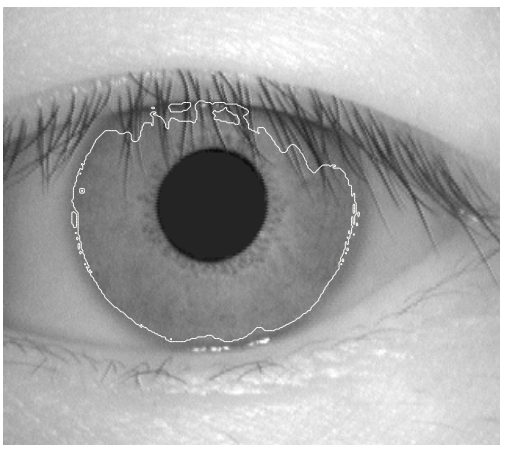

(i)

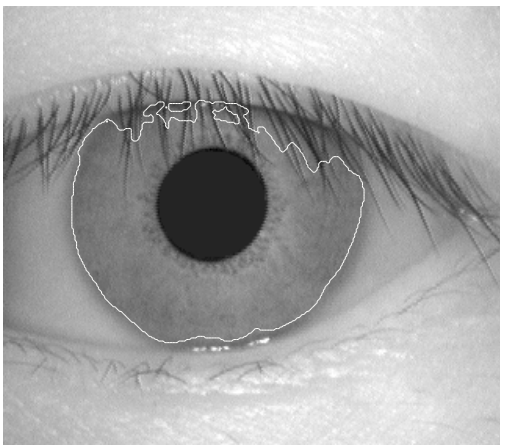

$(\mathrm{k})$

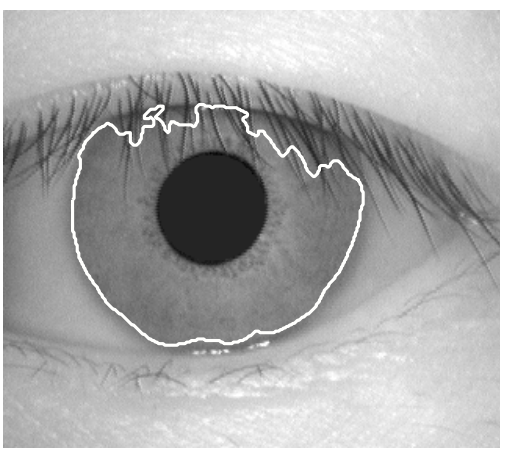

$(\mathrm{m})$

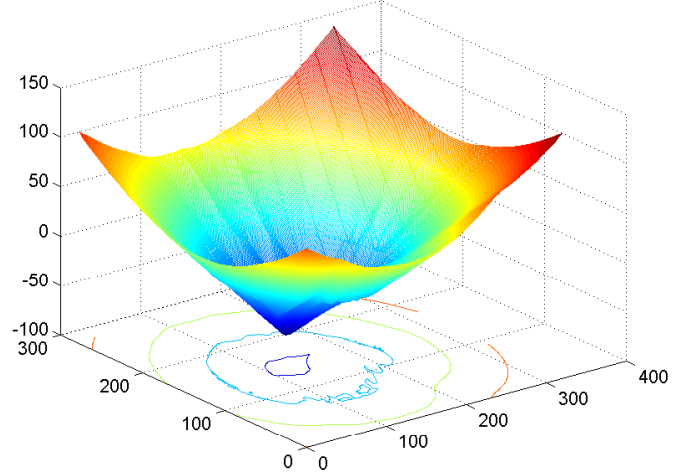

(j)

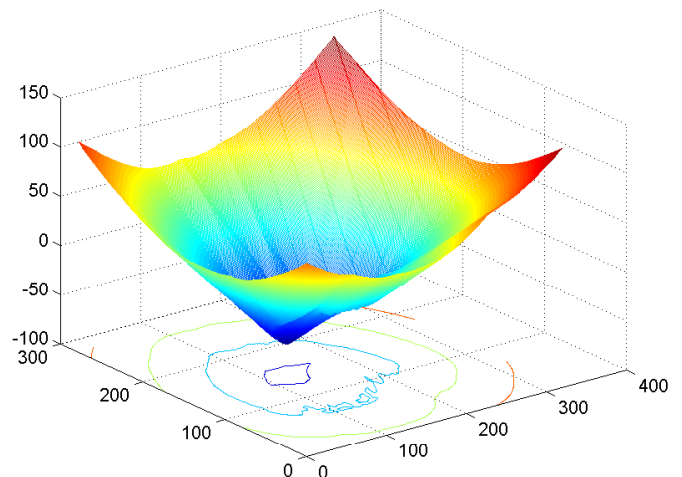

(l)

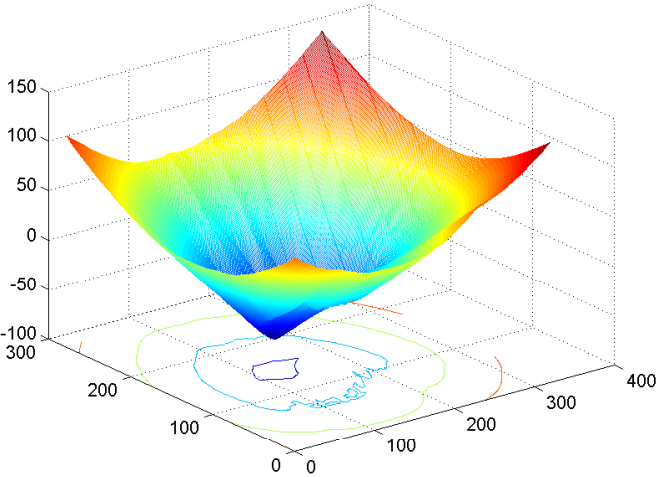

(n)

Figure 2.17: Evolution of the geodesic active contour during iris segmentation.(a) Iris image from CASIA user 5 with initial contour; (b) Embedding function $\psi$ (X and Y axis correspond to the size of the iris image and the $\mathrm{Z}$ axis represents different level sets); (c), (d), (e), (f), (g), (h), (i), (j), (k), (l) Contours after every 100 iterations and their corresponding embedding functions; (m) Final contour after 700 iterations (contours shown using white color). 


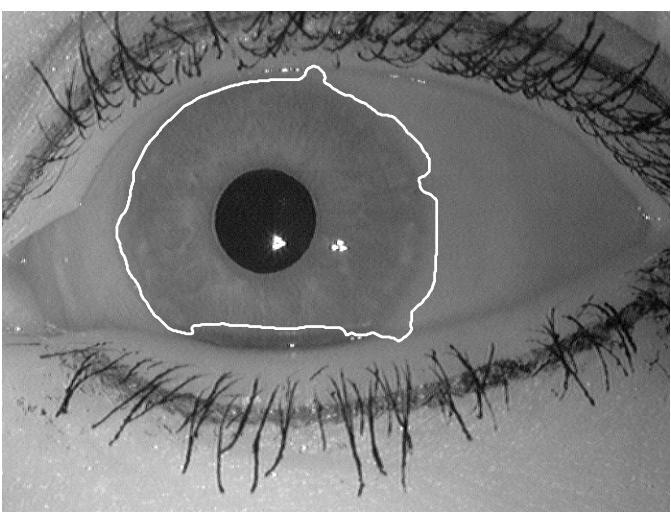

(a)

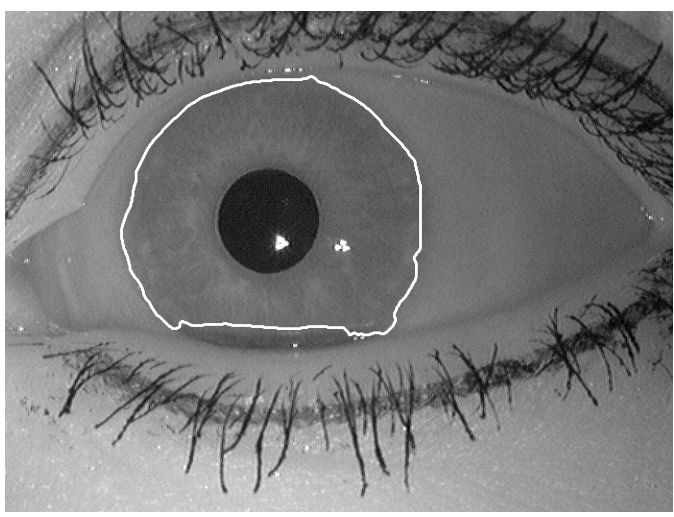

(b)

Figure 2.18: Effect of minimizing the thin plate energy of the levels sets on the geodesic active contour evolution.(a) Contour without minimizing the think plate energy of the level sets; (b) Contour obtained by minimizing the thin plate energy of the level sets (contours shown using white color).

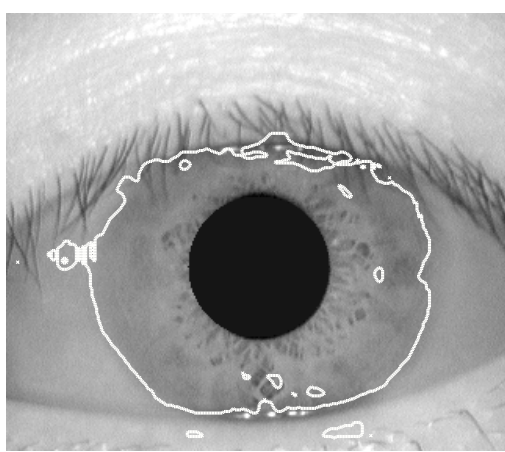

(a)

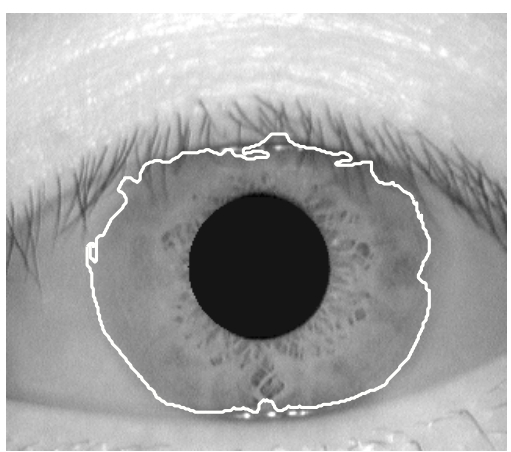

(b)

Figure 2.19: Final contour segmenting the iris.(a) Geodesic contour splitting at various local minima; (b) Final contour (contours shown using white color). 


\section{CHAPTER 2. IRIS SEGMENTATION USING GEODESIC ACTIVE CONTOURS 31}

of $\psi$, we can evolve the embedding function in a narrow band around the zeroth level set [32]. This accelerates the evolution procedure dramatically and, hence, the whole localization and normalization process takes less than 20 seconds (in a MATLAB environment) 1 . To avoid the resulting embedding function from being badly conditioned, the level sets are periodically reinitialized to a signed distance function.

The extracted contour is employed to create the binary mask that is used during the matching of the iriscodes (Equation 2.2). To normalize the iris and convert it to a rectangular entity, its radius and the corresponding center coordinates have to be estimated. If the occlusion due to the upper or lower eyelids is large, then a circle that fits all the points on the extracted contour will lie inside the actual boundary of the iris. Thus, only those points on the contour lying on the boundary of the iris and sclera (as opposed to the iris and the eyelids) should be used to estimate the radius and center of the iris. To ensure this, five points, lying in an arc of $\left[-30^{0}, 30^{\circ}\right]$ and $\left[-150^{\circ}, 150^{\circ}\right]$ with respect to the horizontal axis are randomly selected from the extracted contour and their mean distance from the center of the pupil is computed. This value is used as the approximate radius of the iris $(R)$. A circle is next fitted through all the points on the contour which are within a distance of $R \pm 10$ pixels from the center of the pupil. The center and radius of such a circle is the center $(x, y)$ and the radius $R$ of the iris. Figure 2.20 illustrates the radius of the iris detected by our approach along with the radius of the iris detected using the classical integro-differential operators.

The normalized iris is then pre-processed using the Perona-Malik [47] anisotropic nonlinear diffusion algorithm. The anisotropic diffusion equation of an image $I$ can be written as

$$
I_{t}=\operatorname{div}(g(\nabla I) \nabla I)
$$

where, the edge descriptor $g(\nabla I)$ controls the degree of smoothing at each point in the image $I$ and is given by

$$
g(\nabla I)=\frac{1}{1+\left(\frac{\|\nabla I\|}{k}\right)^{\alpha+1}} \quad(\alpha>0) .
$$

Here $k$ and $\alpha$ are constants. ${ }^{2}$ From the above equation, it is clear that whenever there is no edge

\footnotetext{
${ }^{1} 20$ seconds is the maximum time taken. Depending on the difference between the iris and the pixel radii, this process can take fewer seconds

${ }^{2}$ In our experiments, $k$ is dynamically found such that $90 \%$ of the pixels have gradient magnitude $<k$ and $\alpha=1$
} 


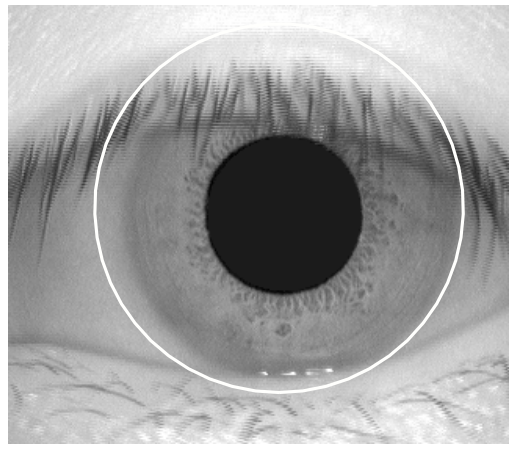

(a)

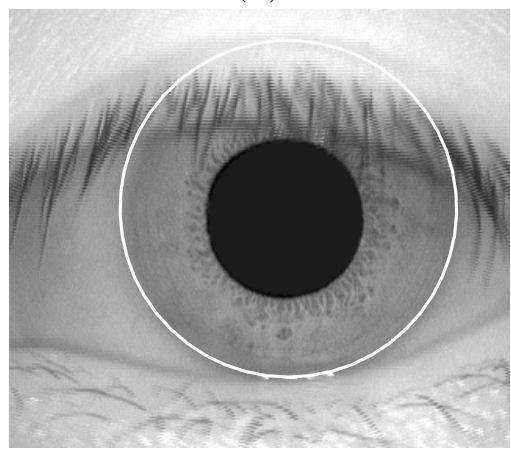

(c)

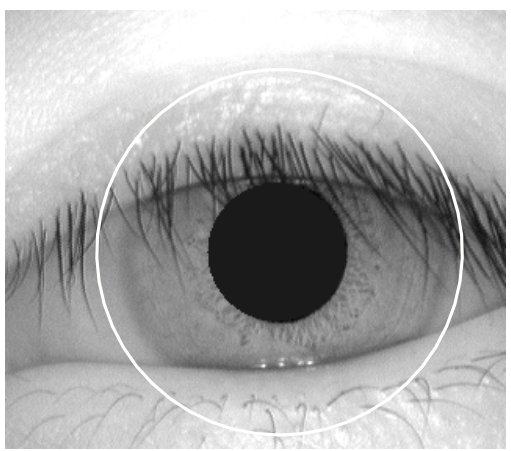

(b)

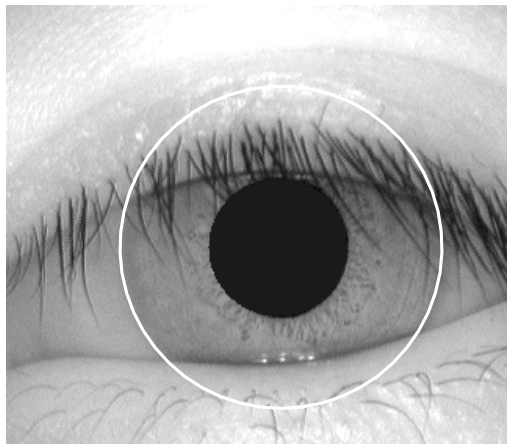

(d)

Figure 2.20: Robust detection of the radius and co-ordinates of the iris using geodesic active contours. (a) and (b) Radius of the iris estimated using circular gradient operators; (c) and (d) Radius of the iris estimated using geodesic active contours (iris radius shown using white color). 


\section{CHAPTER 2. IRIS SEGMENTATION USING GEODESIC ACTIVE CONTOURS33}

in the image, the $g(\nabla I)$ function will approach unity and will act as an isotropic heat diffusion equation; Whereas, the locations where edges are present will have a big $\|\nabla I\|$ value and thus the $g(\nabla I)$ function will be nearly zero causing the edge information to be retained. By applying the anisotropic diffusion algorithm, the noise in the image is expected to be reduced. The enhanced output image is then subjected to histogram equalization. Figure 2.21 (c) shows the normalized image after applying Perona-Malik anisotropic diffusion and histogram equalization.

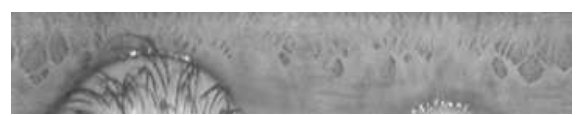

(a)

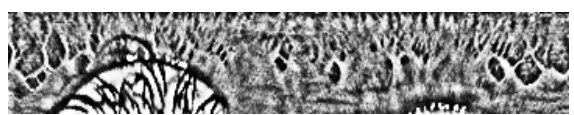

(b)

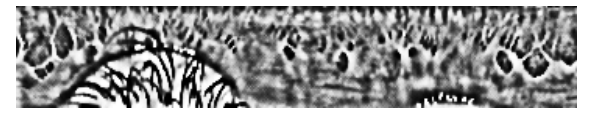

(c)

Figure 2.21: Perona-Malik anisotropic diffusion.(a) Normalized iris image; (b) Normalized iris image enhanced using histogram equalization; (c) Normalized iris image enhanced using anisotropic diffusion and histogram equalization.

\subsection{Analyzing segmentation performance}

The matching performance of our segmentation algorithm was evaluated on the CASIA and WVU non-ideal iris image databases. The CASIA iris database has 756 iris images corresponding to 108 different irises. Each image is of size $320 \times 280$ pixels. The WVU non-ideal iris database has the left (" $L$ ") and right ( $R$ ") irises of 207 individuals ${ }^{3}$. It was noticed that the "L" corresponds to the right eye and the " $\mathrm{R}$ " corresponds to the left eye. There are between $4-15$ images of both the left and right irises of each individual. The total number of images in the WVU non-ideal iris database is 2,678. Each image is of size $640 \times 480$ pixels. Three techniques, viz., geodesic active contours, integro-differential operators and Masek's segmentation technique [34] were used to segment the iris. The encoding and normalization of the iris was then carried out using the algorithm as described in Section 2.2. From the ROC curves for CASIA database (Figure 2.27), it is clear that the matching performance of the iris recognition system significantly improves

\footnotetext{
${ }^{3}$ This is an actively expanding database.
} 


\section{CHAPTER 2. IRIS SEGMENTATION USING GEODESIC ACTIVE CONTOURS34}

when the geodesic active contour scheme is used for segmentation. For example, the GAR (Genuine Acceptance Rate), at a fixed FAR (False Acceptance Rate) of $0.01 \%$ using geodesic active contours for segmentation is 94\% while that using Masek's [34] code is $91.5 \%$ and that using our implementation of Daugman's code is $88 \%$.

The ROC curves for WVU non-ideal left-iris database (Figure 2.23 (a)) indicate that performance does not change by segmenting irises using geodesic active contours as compared to the Masek's segmentation. But a significant performance gain is achieved by segmenting the WVU non-ideal right-iris database using geodesic active contours (Figure 2.23 (b)). For example, the GAR at a fixed FAR of $0.001 \%$ using geodesic active contours for segmentation is $58 \%$ while that using Masek's segmentation is $1 \%$ and using our implementation of Daugman's code is $3 \%$.

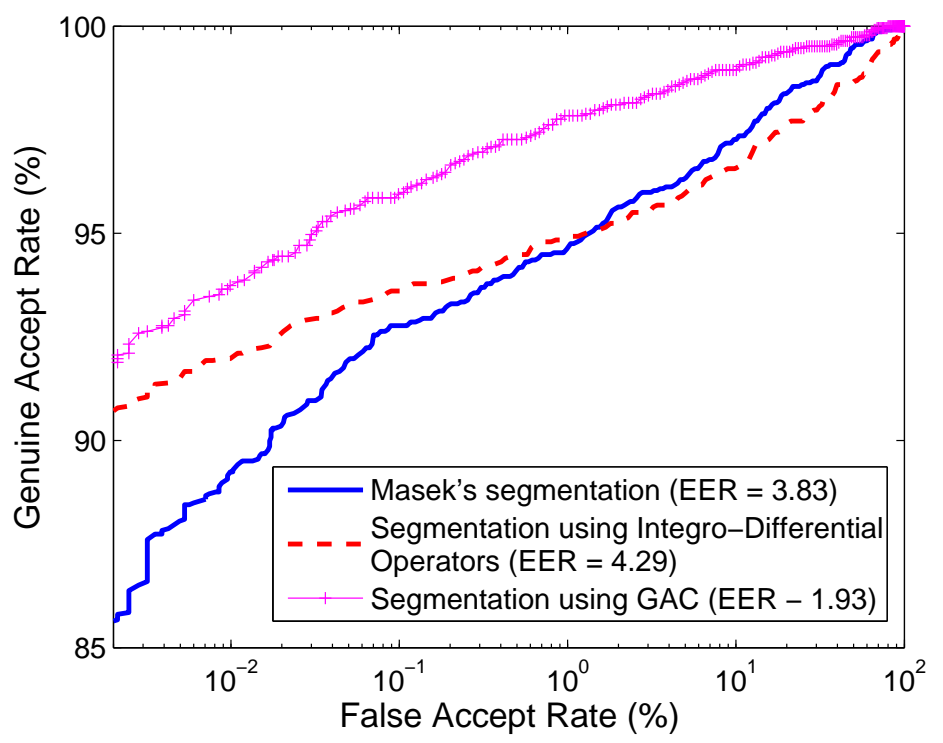

Figure 2.22: Receiver Operating Characteristics for the CASIA iris database.

\subsubsection{A rejection criteria for highly occluded irises}

Upon analyzing the performance, it was observed that many irises in the CASIA database were occluded by the presence of eyelids and eyelashes. Figure 2.24 shows two such examples. Thus the irises, which are occluded to a large extent, do not have sufficient iris information. This is apparent in their corresponding masks (Figure 2.25). To determine the amount of iris information present in the normalized iris images compared to the non-iris regions (like eyelids and eyelashes), a histogram of the percentage of iris texture (unmasked regions) present in the 


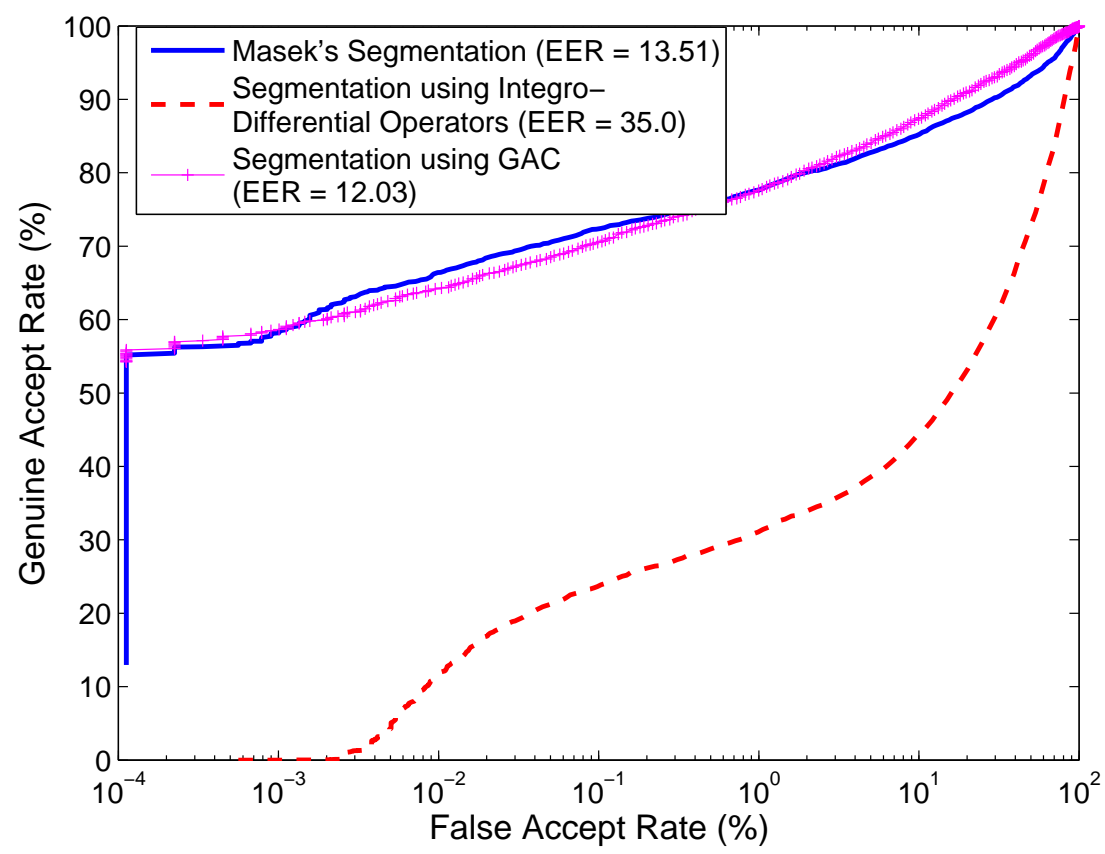

(a)

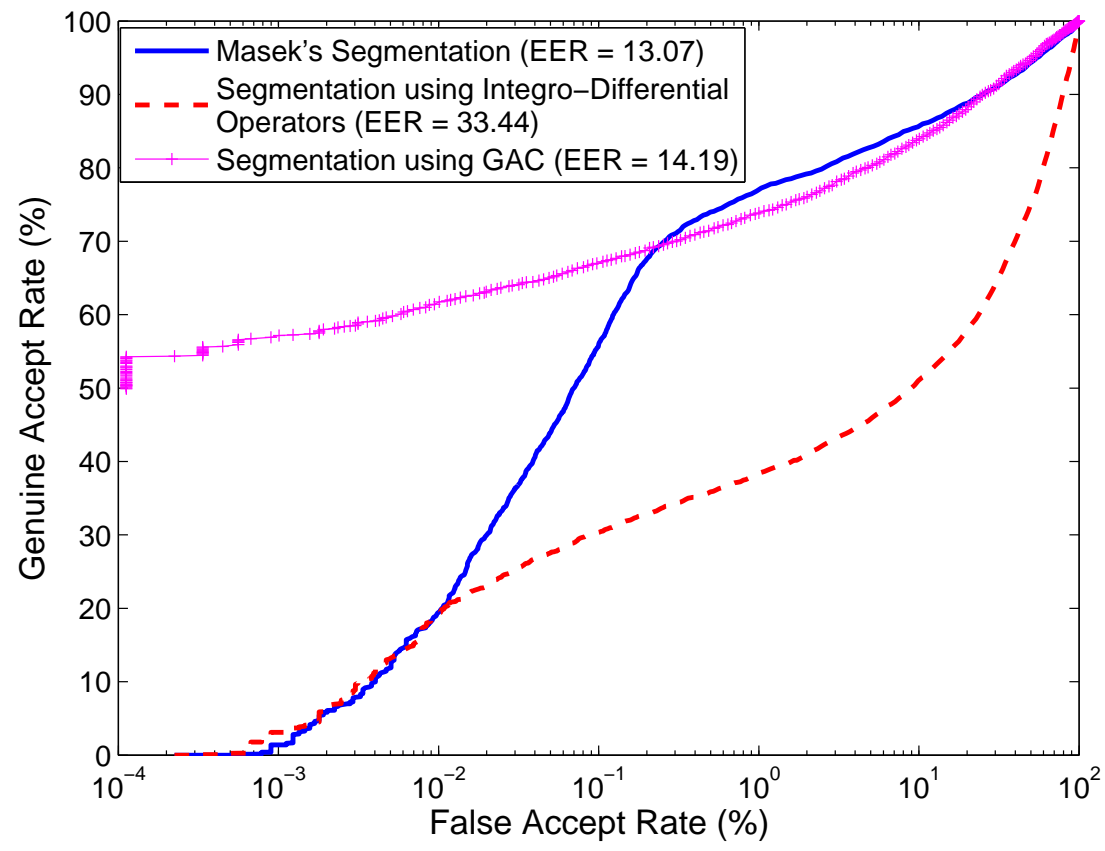

(b)

Figure 2.23: Receiver Operating Characteristics for the WVU non-ideal iris database: (a) Left iris; (b) Right iris. 


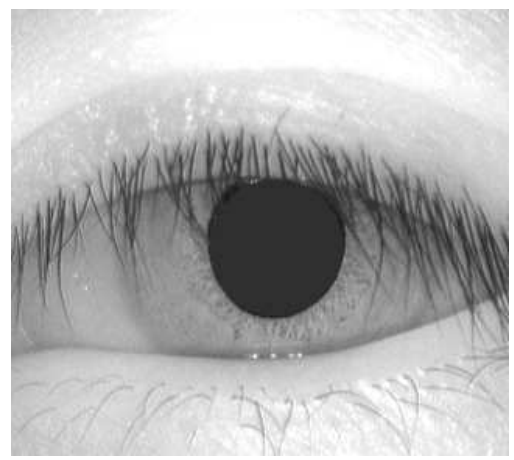

(a)

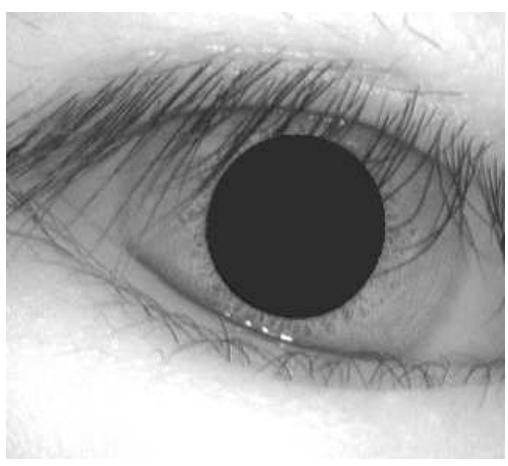

(b)

Figure 2.24: Occluded irises due to eyelids and eyelashes in the CASIA dataset. (a) Occluded iris (user 41); (b) Occluded iris (user 91).

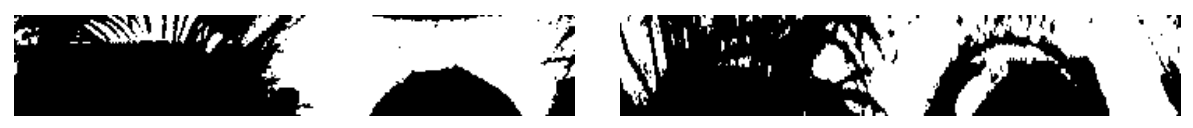

(a)

(b)

Figure 2.25: Masks of the occluded irises in Figure 2.24 due to eyelids and eyelashes.

CASIA database was plotted (see Figure 2.26). It is evident from the histogram in Figure 2.26 that some irises in the CASIA database have very less iris content revealed. In fact, approximately $11 \%$ of all the iris images had iris content less than $67 \%$ of the total normalized image. Figure 2.27 shows the performance of the iris recognition system when such irises are rejected by the system. Examples of such rejected iris images along with their masks are shown in Figures 2.28 and 2.29 , respectively.

Figure 2.30 shows the histogram of the percentage of iris texture (unmasked regions) revealed in the WVU non-ideal right and left iris images. It is evident from the histograms in Figures 2.30 (a) and (b) that not many irises in the WVU non-ideal iris database are occluded. Approximately, only $3 \%$ of all the right iris images and $2 \%$ of the left iris images have iris content less than $67 \%$. Hence rejecting these would not significantly impact the performance of the iris recognition system, which is apparent from the ROC curve in Figure 2.31.

\subsubsection{Using GAC to segment other iris dataset images}

The proposed pupil and iris segmentation algorithms was also tested on the WVU off-angle iris database. This database was collected at the 'Eye Center' at the West Virginia University. 


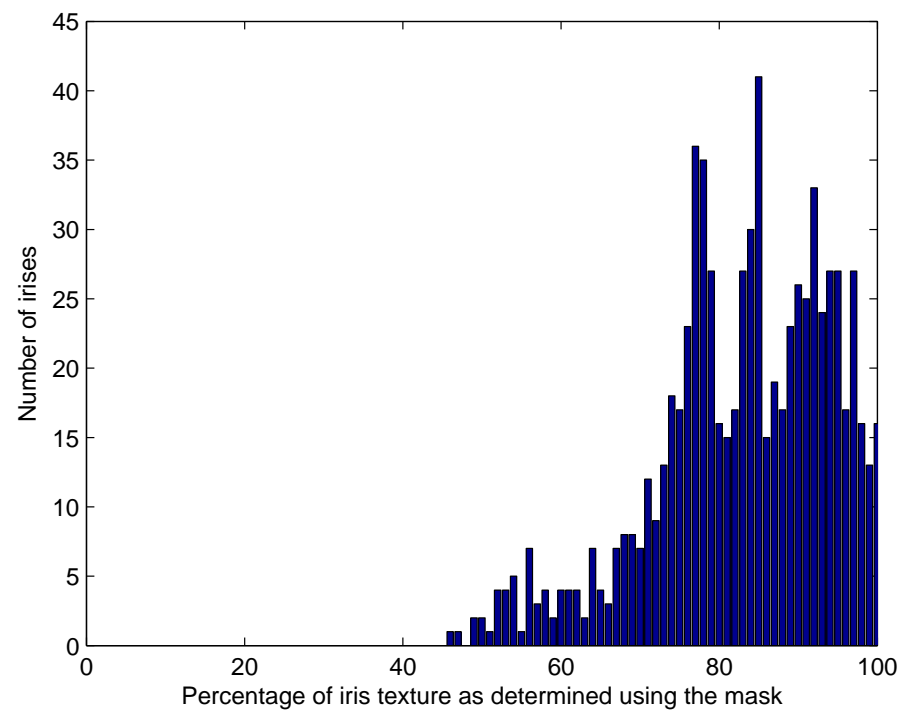

Figure 2.26: Histogram of available iris texture information in the normalized images of CASIA.

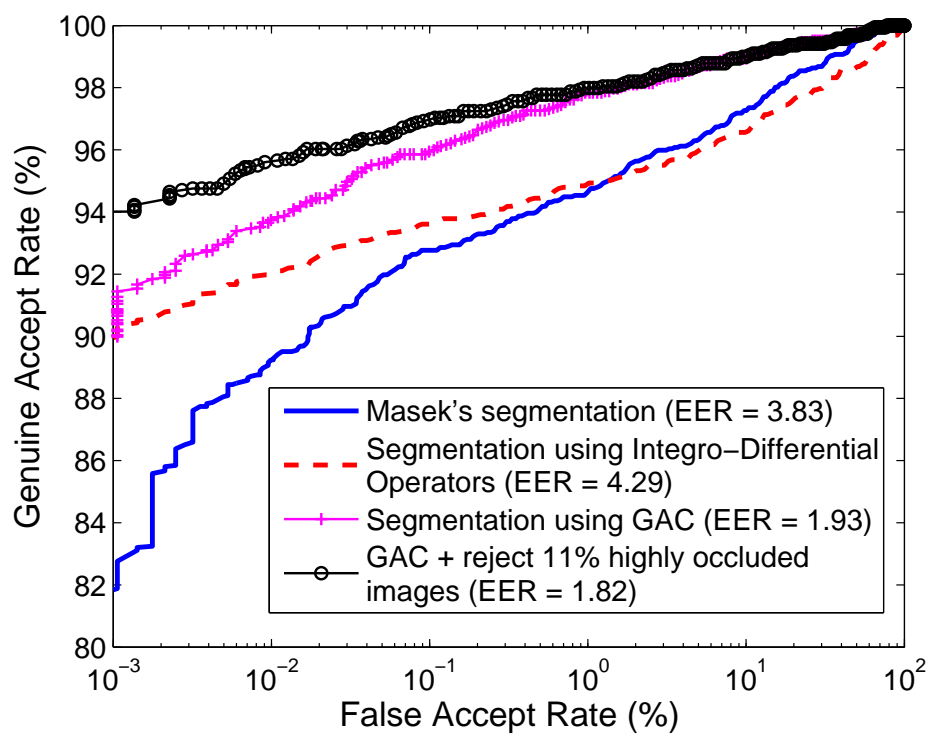

Figure 2.27: ROC curves indicating the performance improvement when iris images (11\%) with very large occlusion are rejected. 

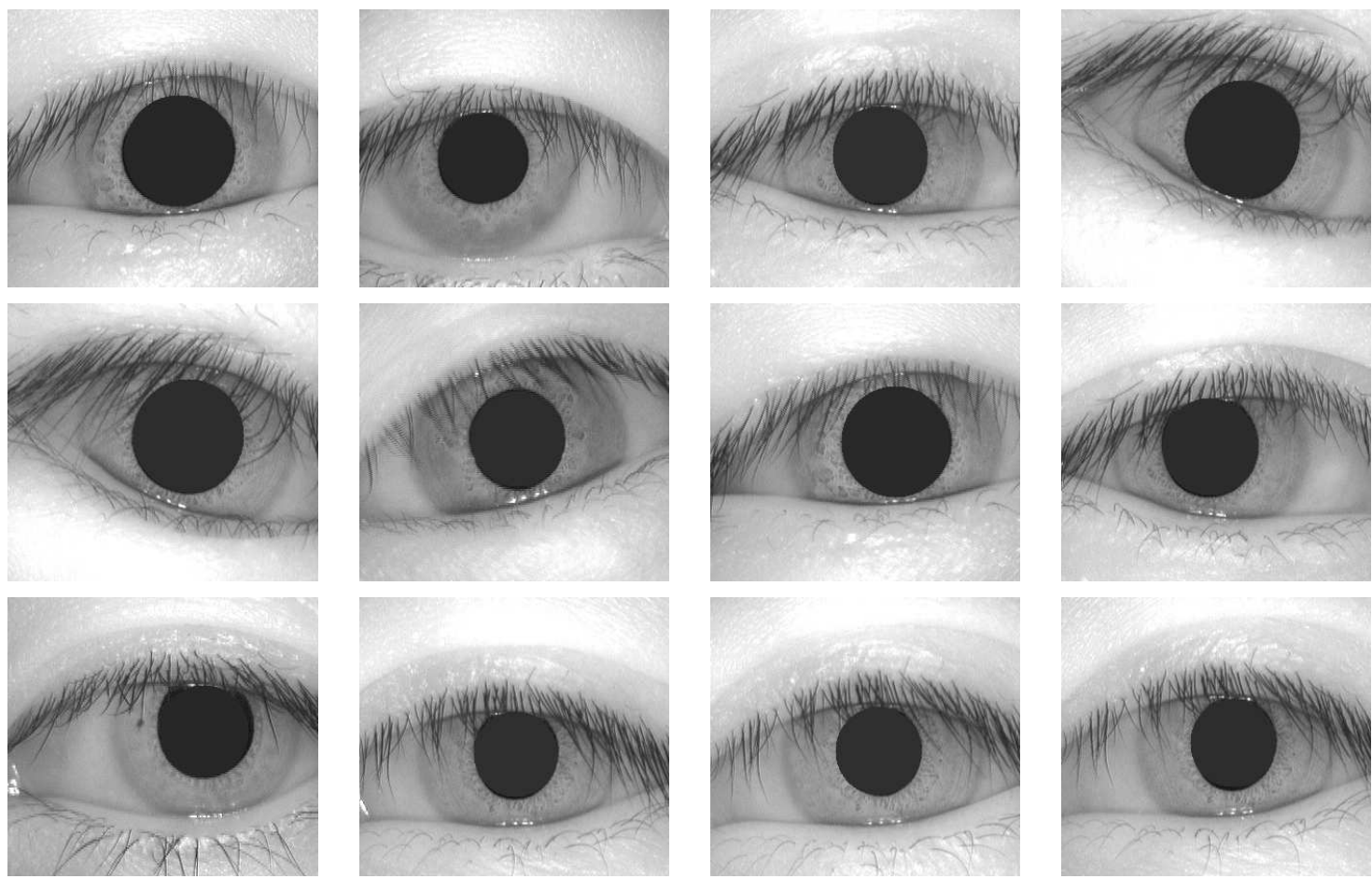

Figure 2.28: Sample irises rejected using the available iris texture information criterion.
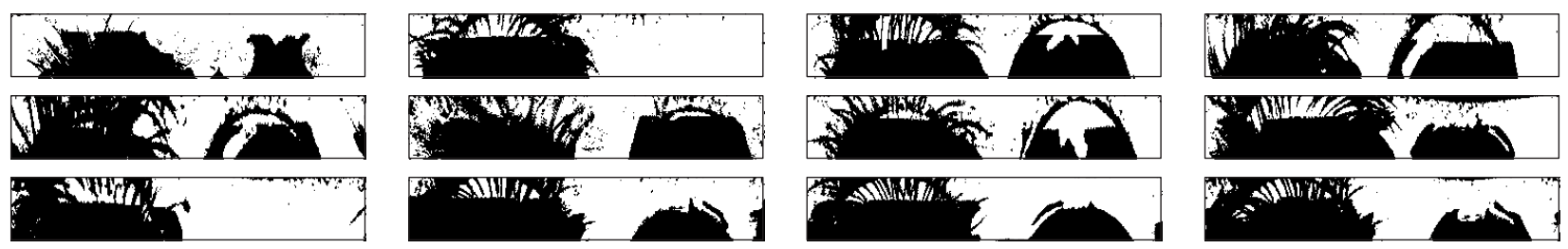

Figure 2.29: Masks of irises rejected in Figure 2.28 using the available iris texture information criterion. 


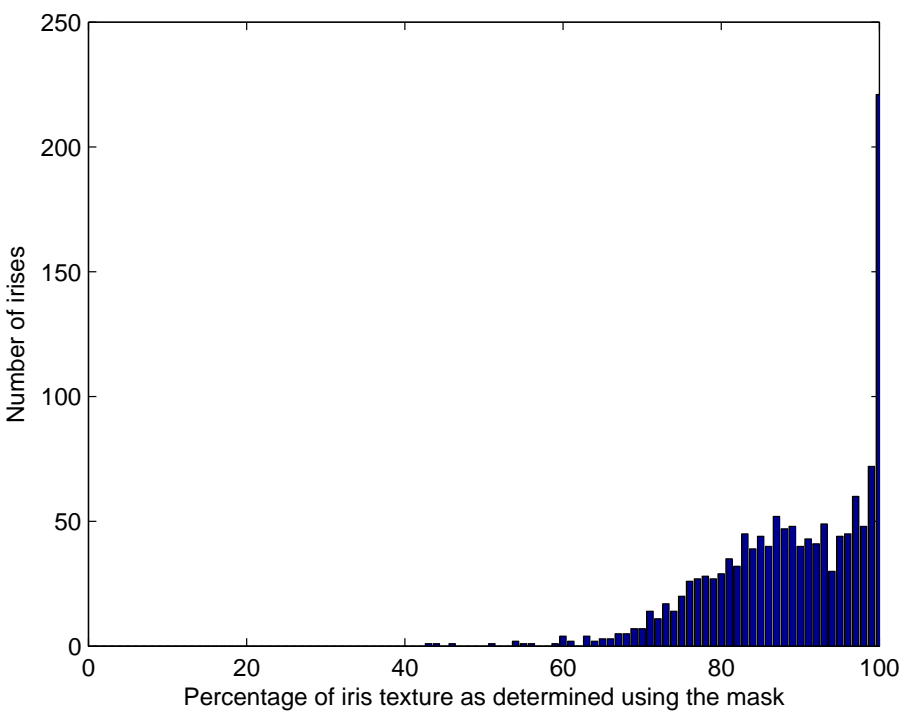

(a)

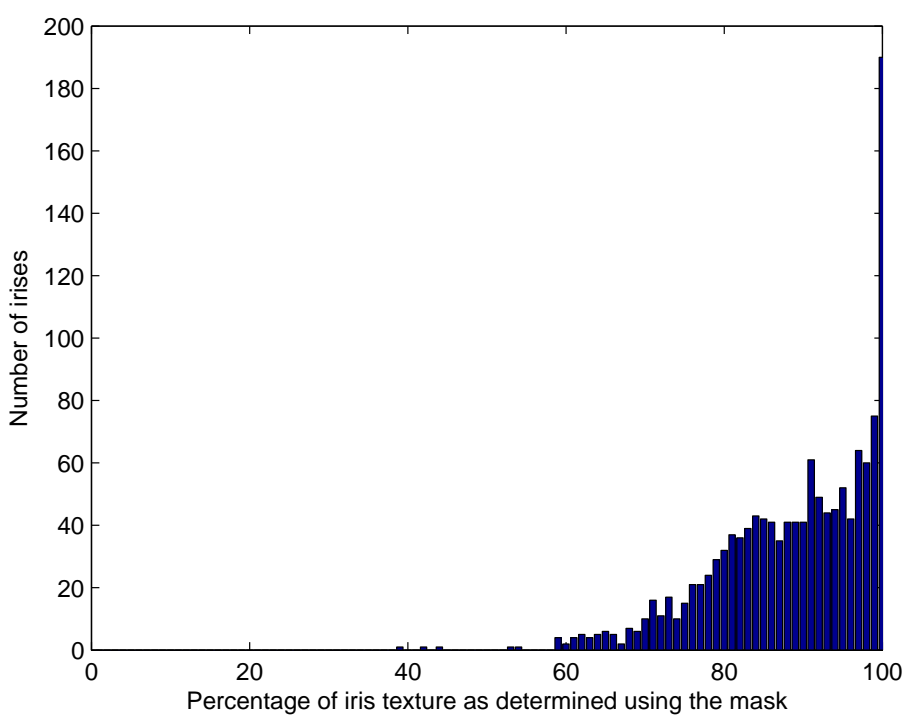

(b)

Figure 2.30: Histogram of available iris texture information in the normalized images of the WVU non-ideal iris database: (a) Right iris; (b) Left iris. 


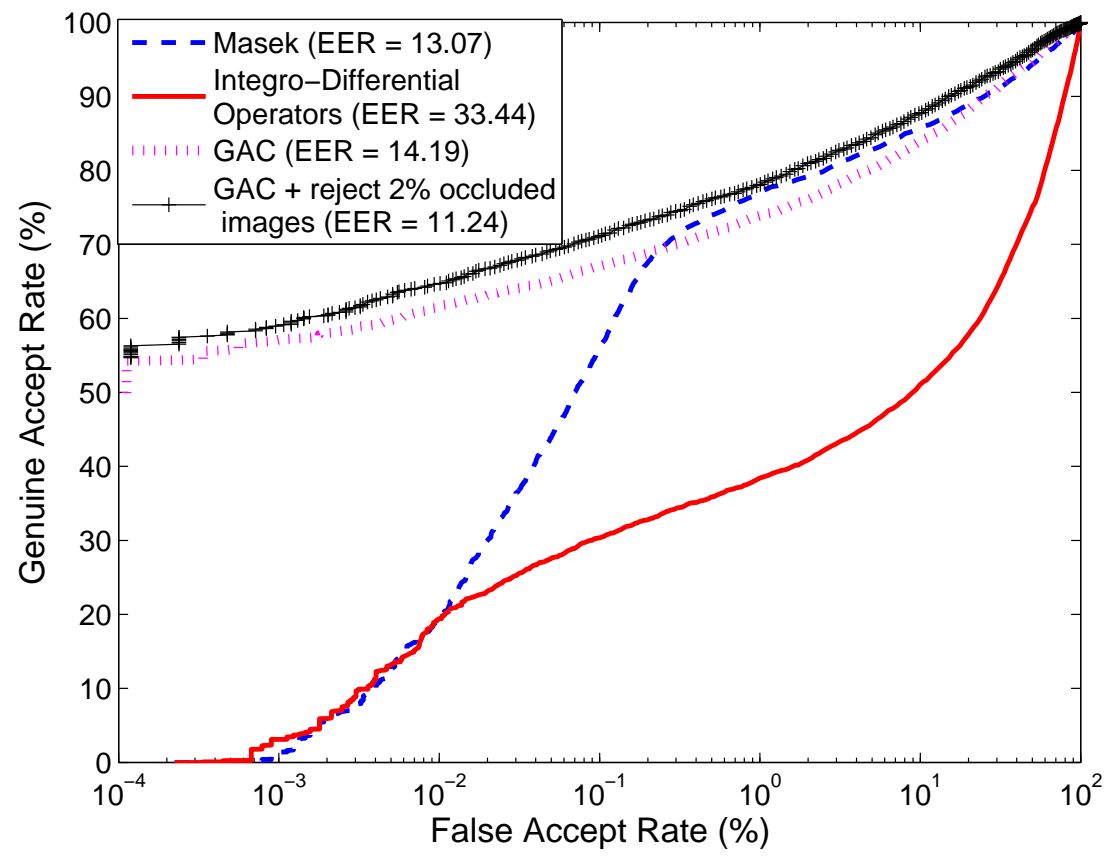

(a)

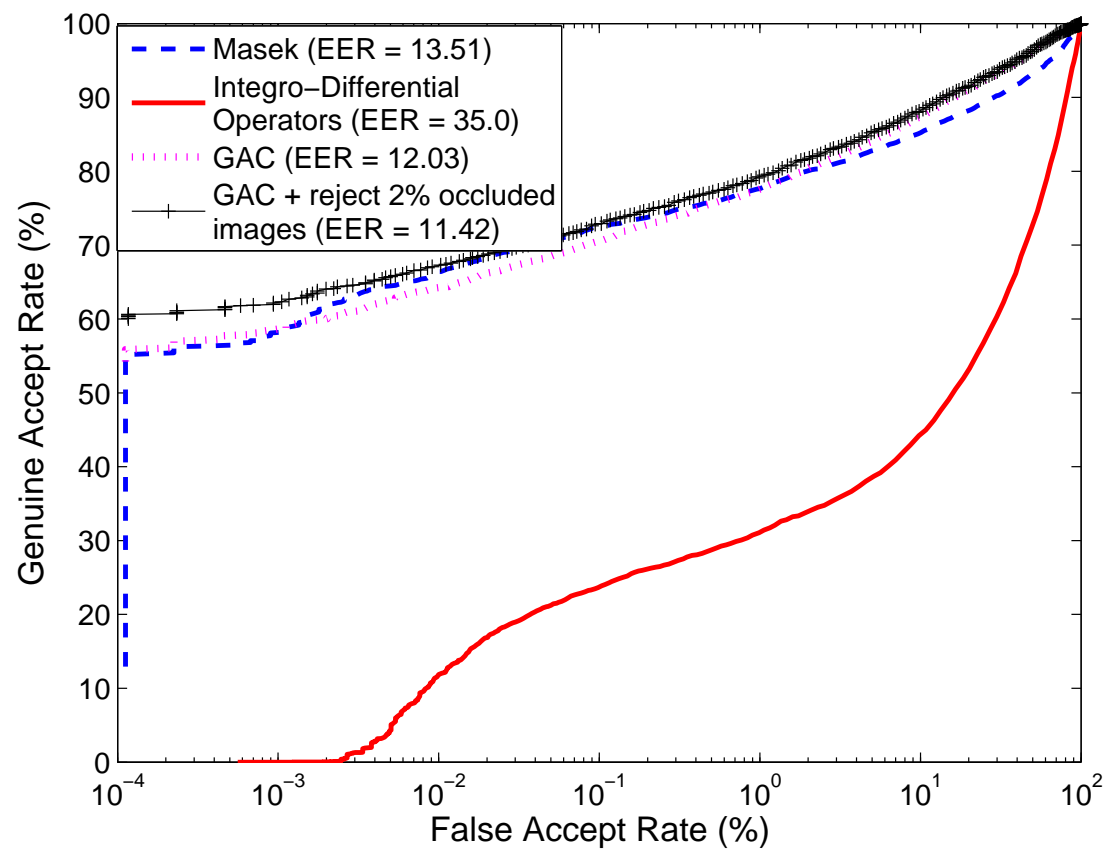

(b)

Figure 2.31: ROC curves indicating the performance improvement when iris images ( $2 \%$ of right irises and 3\% of left irises of WVU non-ideal iris database) with very large occlusion are rejected: (a) Right iris; (b) Left iris. 


\section{CHAPTER 2. IRIS SEGMENTATION USING GEODESIC ACTIVE CONTOURS41}

This database has iris images corresponding to 101 different individuals collected in a single session. 4 images of an individuals left and right iris were taken at an angle of 0,15, 30 and again 0 degrees. Thus a total of 808 iris images corresponding to 202 classes of irises are present. Each image is of size $720 \times 480$ pixels. In our experiments, only a small subset of images were used to evaluate our algorithm. Iris images corresponding to 20 individuals were chosen at random. Thus a total number of 80 images of both the left and right iris were used. The ROC curves for WVU off-angle right-iris database (Figure 2.32 (a)) indicate that a significant performance gain is achieved by segmenting the WVU off-angle iris database using geodesic active contours. For example, the GAR at a fixed FAR of $0.1 \%$ using geodesic active contours for segmenting right-irises is $40 \%$ while that using Masek's segmentation is $1 \%$ and using our implementation of Daugman's code is 18\%. Similarly, the GAR at a fixed FAR of $0.1 \%$ using geodesic active contours for segmenting left-irises is $50 \%$ while that using Masek's segmentation is $28 \%$ and using our implementation of Daugman's code is $8 \%$.

The proposed pupil and iris segmentation algorithms were also used to segment images of a few other databases such as the UBIRIS database [48] and the $M M U 1$ iris database [49]. The UBIRIS database is collected in two separate sessions. A Nikon E5700 camera was used to capture RGB color images. Each image is of size $800 \times 600$ pixels. 241 subjects participated in the first session and 5 images per subject were taken. Only 132 of the 241 subjects participated in the second session and again 5 images per iris were taken. Thus, the UBIRIS database consists of a total of 1,877 iris images. The $M M U 1$ iris database was captured using a LG IrisAccess®2200 camera. It consists of iris images of 45 subjects. 5 images each of the left and the right eye were taken of every individual. Thus, the $M M U 1$ iris database consists of a total of 450 iris images with 90 classes. The size of the images is $320 \times 280$ pixels. The segmentation results on WVU off-angle iris images can be seen in Figure 2.33, on the UBIRIS images in Figure 2.34 and on the $M M U 1$ iris images in Figure 2.35.

Tables 3.1, 2.3, 2.4 summarize the performance improvement using geodesic active contours for segmentation for CASIA, and WVU non-ideal (left and right iris) iris images, respectively. 


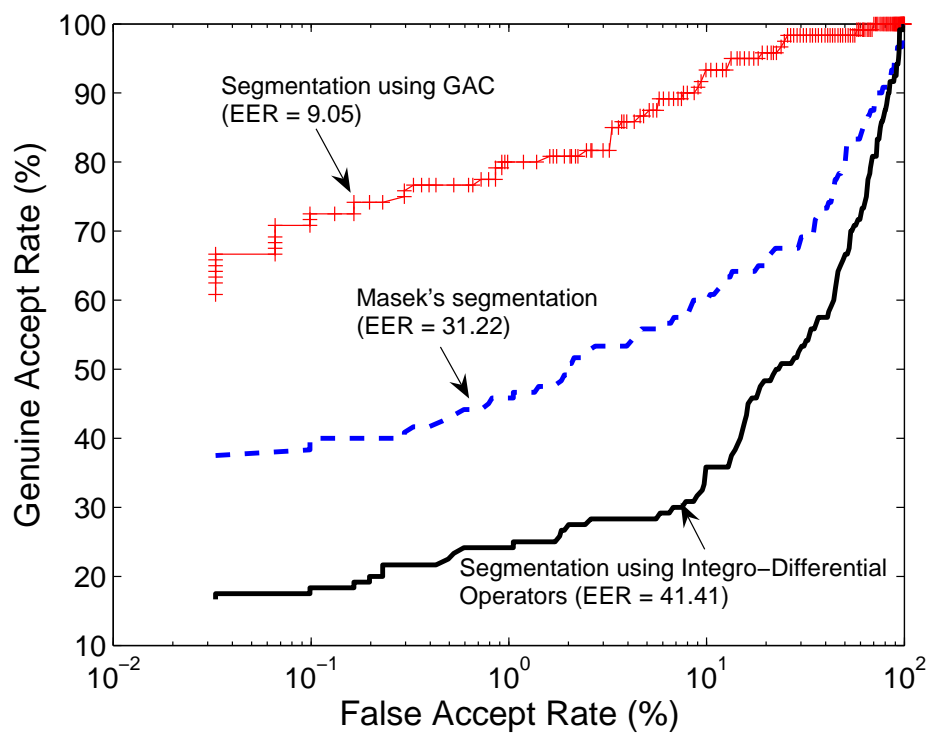

(a)

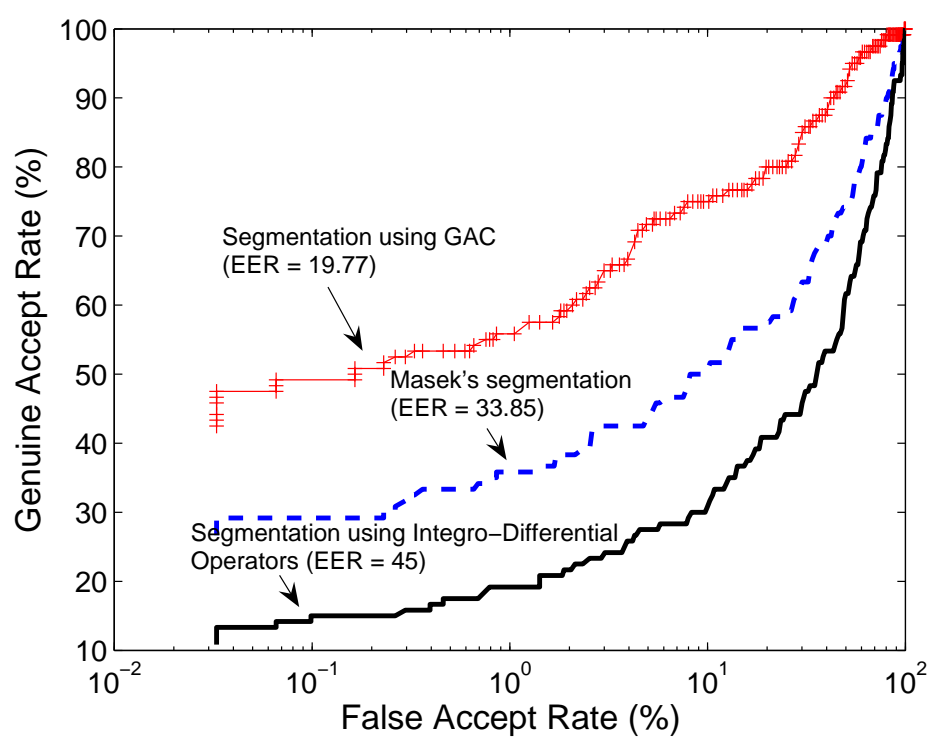

(b)

Figure 2.32: Receiver Operating Characteristics for the WVU off-angle iris database: (a) Right iris; (b) Left iris. 
CHAPTER 2. IRIS SEGMENTATION USING GEODESIC ACTIVE CONTOURS 43
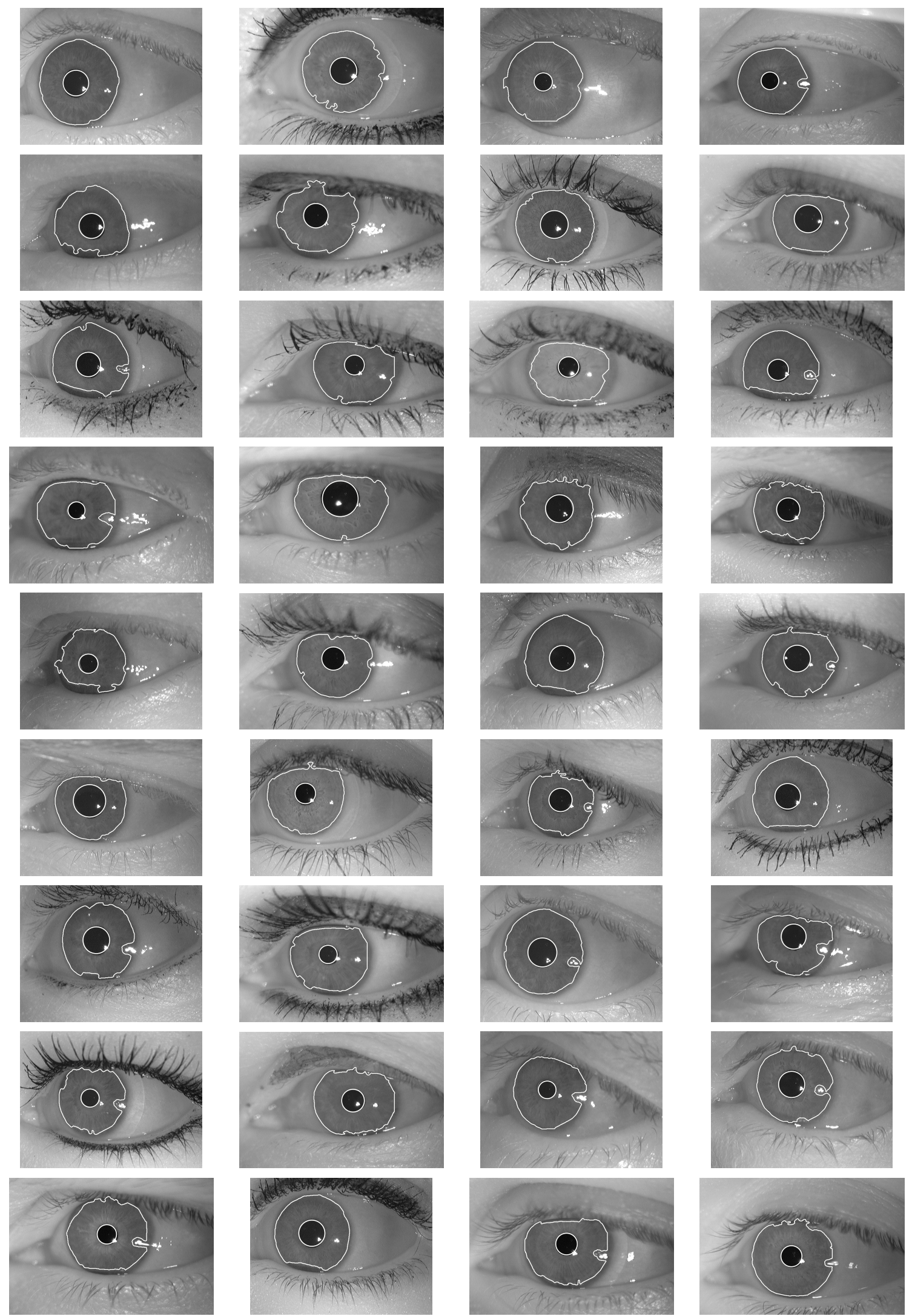

Figure 2.33: Segmenting WVU Non-Ideal irises using the proposed algorithm. 
CHAPTER 2. IRIS SEGMENTATION USING GEODESIC ACTIVE CONTOURS44
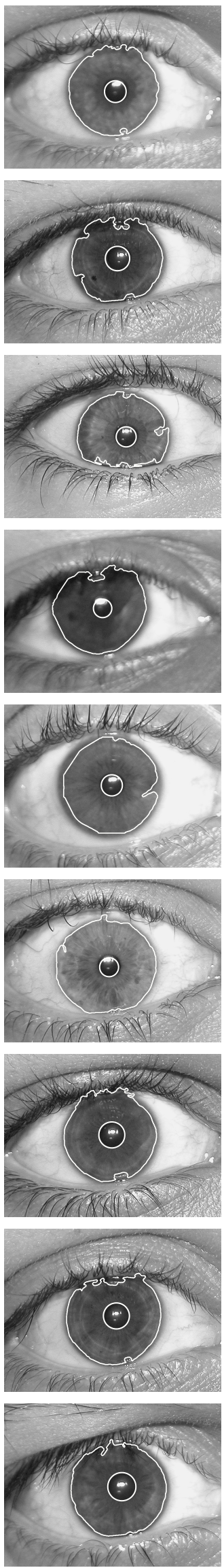
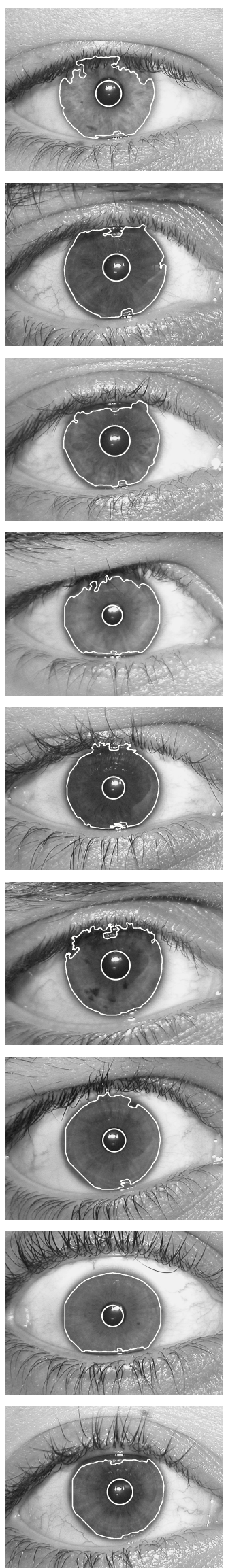
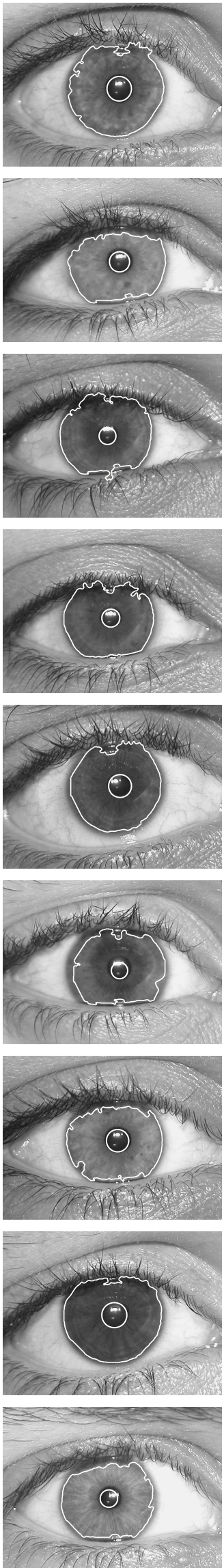
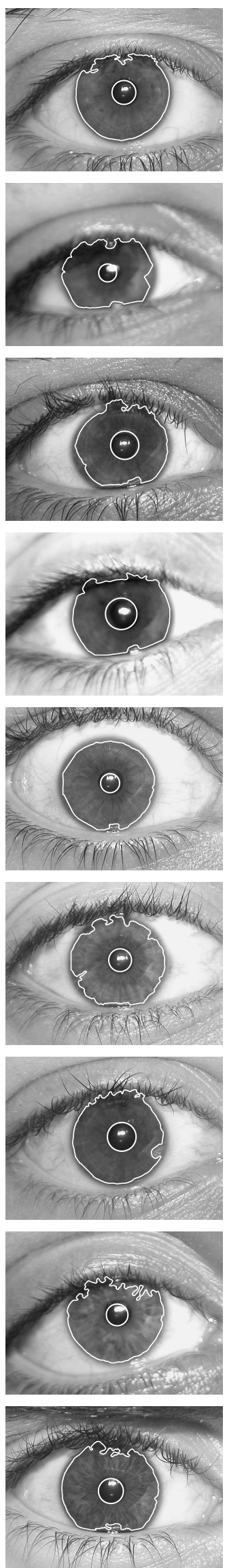

Figure 2.34: Segmenting UBIRIS irises using the proposed algorithm. 
CHAPTER 2. IRIS SEGMENTATION USING GEODESIC ACTIVE CONTOURS45
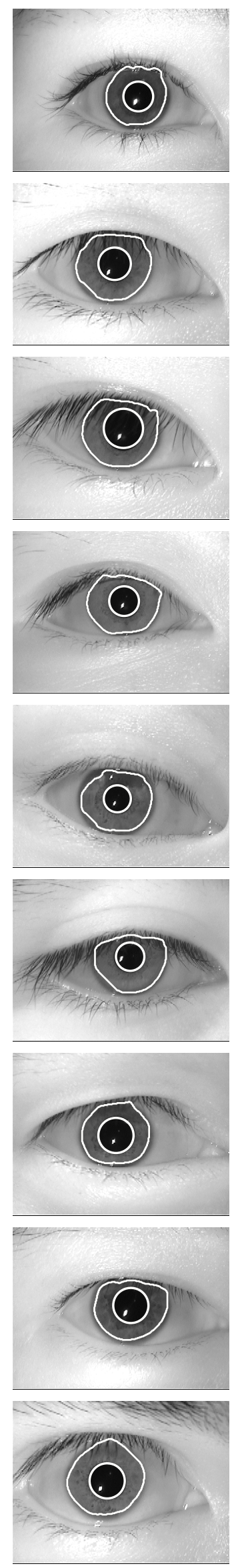
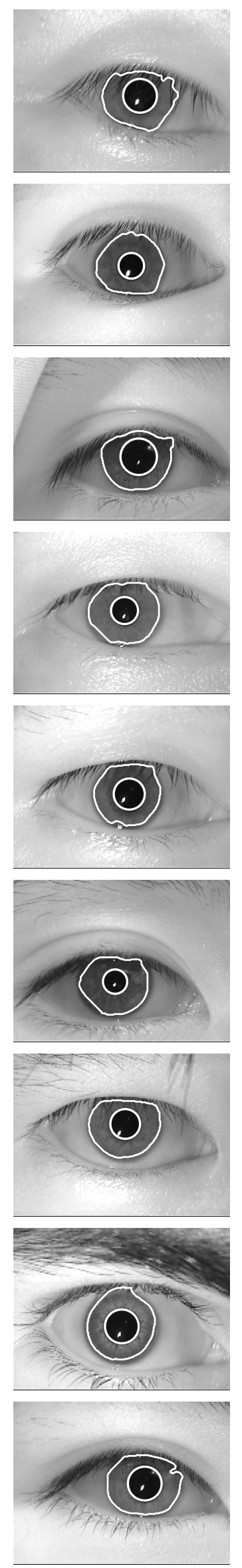
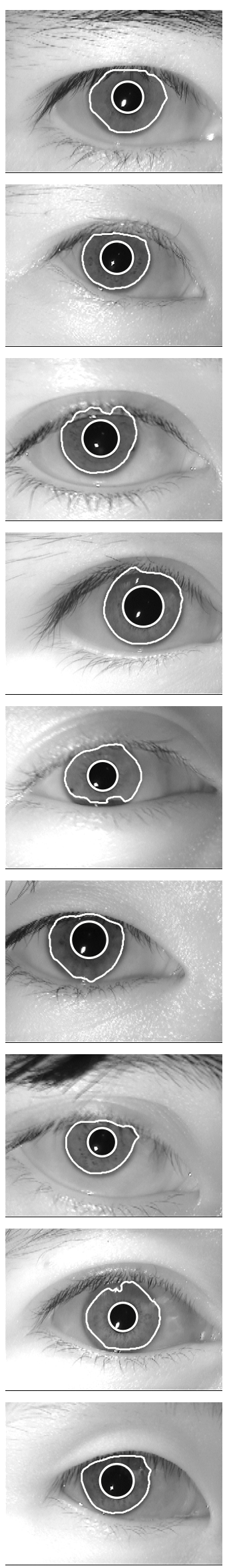
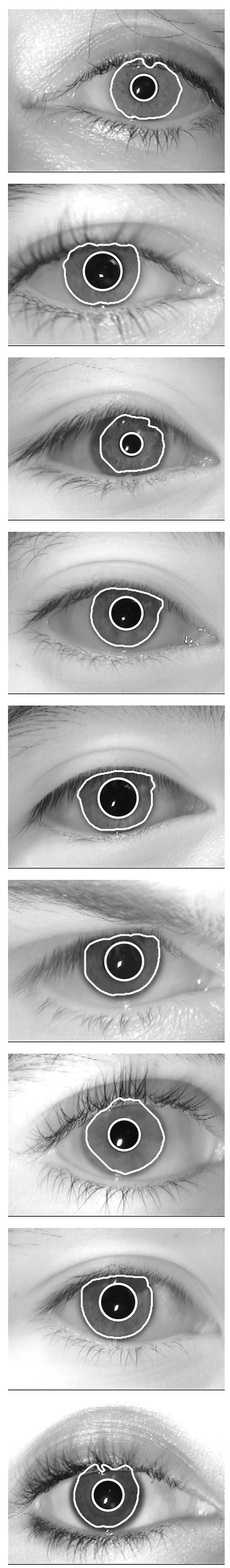

Figure 2.35: Segmenting $M M U 1$ irises using the proposed algorithm. 


\begin{tabular}{|l|l|l|l|l|}
\hline FAR (\%) & \multicolumn{3}{|c|}{ GAR (\%) } \\
\hline & $\begin{array}{l}\text { Masek's } \\
\text { code }\end{array}$ & Integro-differential operators & GAC & $\begin{array}{l}\text { GAC and reject 11\% of } \\
\text { highly occluded irises }\end{array}$ \\
\hline 1 & 94.0 & 94.2 & 97.8 & 98.0 \\
\hline 0.1 & 92.6 & 93.8 & 96.0 & 97.0 \\
\hline 0.01 & 88.0 & 91.5 & 94.0 & 95.8 \\
\hline 0.001 & 82.0 & 90.0 & 90.0 & 94.0 \\
\hline
\end{tabular}

Table 2.2: The GAR at a fixed FAR for the CASIA iris database using Masek's code, integrodifferential operators and geodesic active contours (GAC) for iris segmentation.

\begin{tabular}{|l|l|l|l|l|}
\hline FAR (\%) & \multicolumn{4}{|c|}{ GAR (\%) } \\
\hline & $\begin{array}{l}\text { Masek's } \\
\text { code }\end{array}$ & Integro-differential operators & GAC & $\begin{array}{l}\text { GAC and reject 3\% of } \\
\text { highly occluded irises }\end{array}$ \\
\hline 1 & 76.0 & 38.0 & 74.0 & 78.0 \\
\hline 0.1 & 56.0 & 30.0 & 67.0 & 72.0 \\
\hline 0.01 & 20.0 & 20.0 & 62.0 & 65.0 \\
\hline 0.001 & 2.0 & 3.0 & 57.0 & 59.0 \\
\hline 0.0001 & 0.0 & 0.0 & 50.0 & 55.0 \\
\hline
\end{tabular}

Table 2.3: The GAR at a fixed FAR for the WVU non-ideal right-iris database using Masek's code, integro-differential operators and geodesic active contours (GAC) for iris segmentation.

\begin{tabular}{|l|l|l|l|l|}
\hline FAR (\%) & \multicolumn{4}{|c|}{ GAR (\%) } \\
\hline & $\begin{array}{l}\text { Masek's } \\
\text { code }\end{array}$ & Integro-differential operators & GAC & $\begin{array}{l}\text { GAC and reject 2\% of } \\
\text { highly occluded irises }\end{array}$ \\
\hline 1 & 77.0 & 32.0 & 77.0 & 79.0 \\
\hline 0.1 & 72.0 & 24.0 & 71.0 & 73.0 \\
\hline 0.01 & 66.0 & 12.0 & 64.0 & 67.0 \\
\hline 0.001 & 57.0 & 0.0 & 58.0 & 63.0 \\
\hline 0.0001 & 13.0 & 0.0 & 54.0 & 61.0 \\
\hline
\end{tabular}

Table 2.4: The GAR at a fixed FAR for the WVU non-ideal left-iris database using Masek's code, integro-differential operators and geodesic active contours (GAC) for iris segmentation. 


\section{CHAPTER 2. IRIS SEGMENTATION USING GEODESIC ACTIVE CONTOURS47}

\subsection{Discussion}

The process of segmenting the iris plays a pivotal role in iris recognition systems. In this chapter, a technique to enhance the performance of an iris recognition system by modifying the structure of differences in iriscodes has been presented. This novel scheme examines the blockwise structure of differences in iriscodes in order to compare two irises. This can be extremely helpful when accurate iris segmentation is not done. Traditionally, systems have employed the integro-differential operator or its variants to localize the spatial extent of the iris. In this chapter, a novel scheme using geodesic active contours (GAC) for iris segmentation, has been discussed. The GAC scheme is an evolution procedure that attempts to elicit the limbic boundary of the iris as well as the contour of the eyelid in order to isolate the iris texture from its surroundings. Experimental results on the CASIA, WVU non-ideal and the WVU off-angle iris dataset clearly indicate the benefits of the proposed algorithm. The algorithm also aids in accurately estimating the radius of the iris and its center. The results of this segmentation technique have been shown on various databases which suggests that this algorithm is very robust irrespective of the resolution and condition in which the image is captured. Geodesic active contours take relatively more time to segment the iris as compared to other techniques mentioned in the literature. The whole process of segmentation can be accelerated by using a simple multi-resolution approach. The evolution process can be first conducted on a small resolution image to determine the approximate position of the iris. Subsequently, the location details of the iris can be made precise by using higher resolution images and commencing the evolution from the coarser details of the iris. This process can be repeated until the evolution is performed on the highest resolution image (input image) and thereby obtaining the same level of accuracy in locating the iris but dramatically reducing the required time.

Some parameters in the geodesic active contour formulation (Equations 2.8, 2.7) vary across images of different iris databases. The value of these parameters is shown in table 2.5.

The advection force $(c)$, as described earlier, determines how fast the evolving curve has to be pushed outwards. It can have any value between 0 and 1 . The parameters $k$ and $\alpha$ of the stopping function (equation 2.7) depend on the intensity and the contrast of the iris image. These values must be decided in such a way that the stopping function yields most of the edges of the iris. Kalka et al. [50] find the global quality of the iris image by determining the defocus blur, motion blur, occlusion, specular reflection, etc,. These quality factors can be used in order to 
CHAPTER 2. IRIS SEGMENTATION USING GEODESIC ACTIVE CONTOURS 48

\begin{tabular}{|l|l|l|l|}
\hline Parameter & \multicolumn{3}{|c|}{ GAR (\%) } \\
\hline & CASIA & WVU non-ideal & WVU off-angle \\
\hline$c($ advectionforce $)$ & 0.65 & 0.65 & 0.65 \\
\hline$k$ (stoppingfunction $)$ & 1.6 & 2.8 & 2.8 \\
\hline$\alpha$ (stoppingfunction $)$ & 10 & 8 & 8 \\
\hline Maximum iterations & 700 & 1800 & 1800 \\
\hline
\end{tabular}

Table 2.5: Parameters in iris segmentation using Geodesic Active Contours.

dynamically determine the value of the parameters $k$ and $\alpha$ of the stopping function. Also, the local quality estimates ( [51]) can also be used to determine the value of these parameters locally. The upper limit on the number of iterations required for the evolution of the contour depends on the size of the image and also on the radius of the iris being captured by the acquisition device. 


\section{Chapter 3}

\section{Multi-Instance, Multi-Unit Fusion}

\subsection{Limitations of using single iris image}

The performance of an iris recognition system is highly dependant on the quality of the acquired iris image. The iris image quality may be poor due to several reasons.

1. Specular reflections due to the illumination provided by the light emitting diodes.

2. Motion blur resulting either from the relative motion of the object or of the camera during the exposure time.

3. Defocus blur due to the focal point being outside the "depth of field" of the object being captured.

4. Off-Angle images captured from a profile view causing the iris and pupil to be elliptically projected.

5. Occlusion due to the eyelids and the eyelashes.

6. Highly off-centered pupil caused due to some medical problems.

7. Use of contact lenses on which characters are imprinted, e.g., a company's name or logo.

8. Ambient light reflections on the captured iris image.

These poor quality iris images may hamper the performance of the best available iris recognition systems. Figure 3.1 shows some of the common difficulties encountered in capturing good quality iris images. 


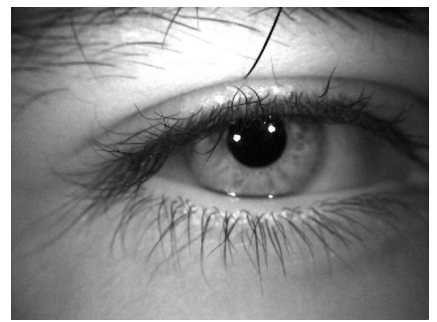

(a) Eyelid occlusion

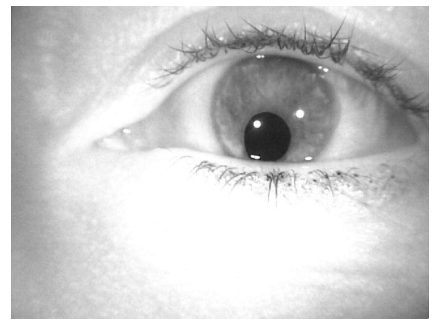

(d) Off-centered pupil

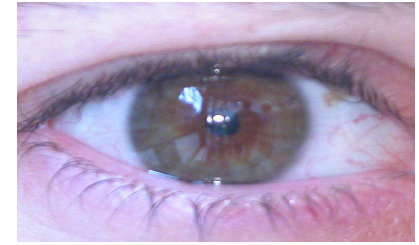

(g) Specular reflection

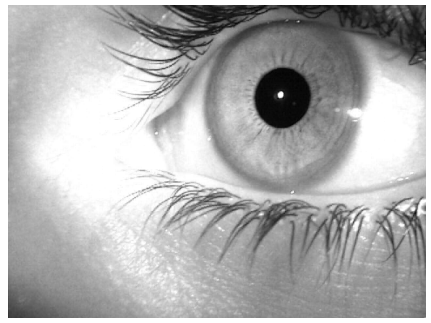

(b) Off-angle iris

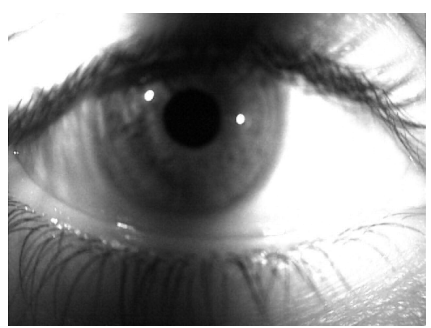

(e) Shadow on iris

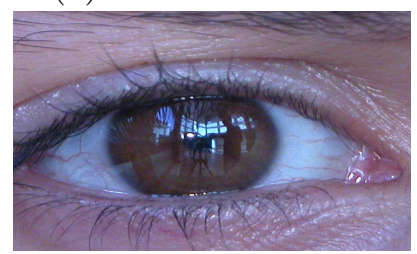

(g) Ambient reflection

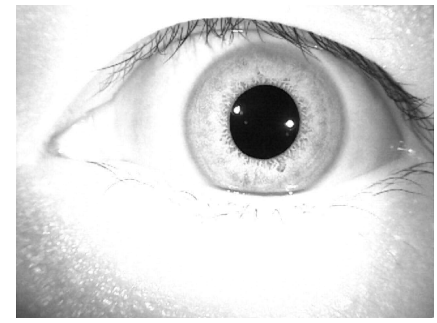

(c) Over exposure

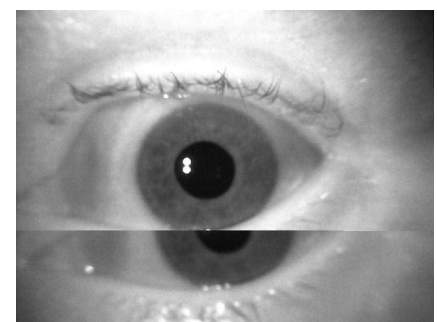

(f) Motion artifact

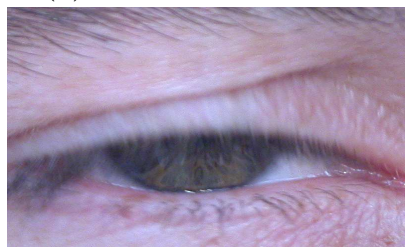

(i) Severely occluded iris

Figure 3.1: Common problems encountered during the image acquisition process. 
Poor quality of iris images cause high Failure To Enrol (FTE) and Failure To Acquire (FTA) rates. Results of the iris recognition test conducted by the International Biometric Group (IBG) show that the failure to enroll rates are inversely related to the algorithm's matching abilities [52]. Thus, the device which had the least FTE (1.61\%), had the highest False Match Rate (FMR) and the False Non Match Rate (FNMR).

Also, some individuals wear contact lenses which have certain characters representing either the company's name which manufactured it or its logo printed on the lens. Figure 3.2 shows an example of an iris with characters "AV" printed on its lens. Such iris images can cause difficulty during the iris matching process.

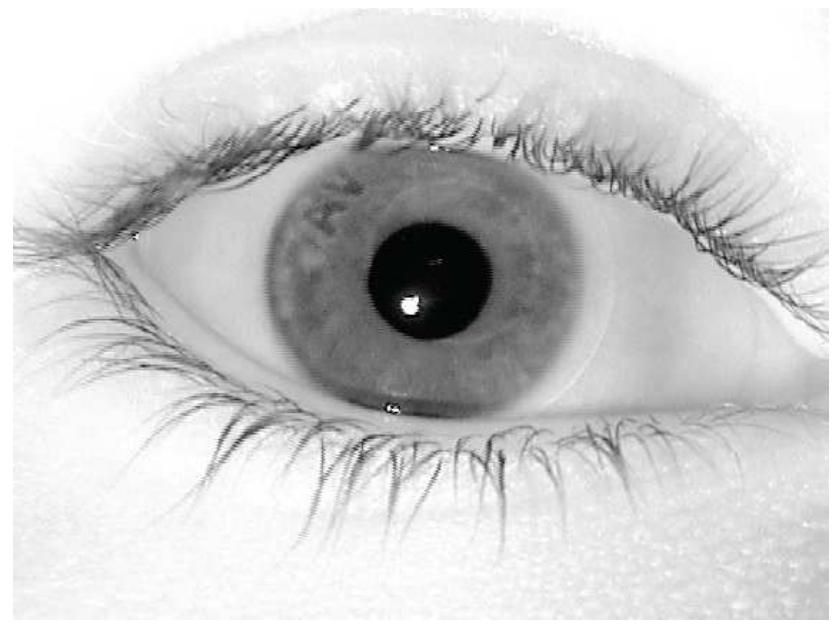

Figure 3.2: An iris image showing a contact lens having the characters "AV" imprinted on it.

In order to address this problem, multiple impressions of the same iris or multiple irises (left and right) can be used to enhance the performance of an iris recognition system.

\subsection{Fusion in iris systems}

In the literature, there are very few algorithms which use either multiple instances of the same iris or both irises (left and right) of a user to improve the performance of an iris recognition system. Jang et al. ( [53], [54]) propose a multi-unit iris recognition system in which after capturing both the left and right irises, the quality of images is checked and the better quality iris image is selected. The Panasonic iris recognition system [55] also captures both eye images at the same time and the user is accepted only if either of the two iris images can be identified. There 
have also been a few instances in literature which use multiple algorithms to recognize irises. Sun et al. [56] use a cascading classifier scheme to combine the statistical iris features with the structural ones. First the statistical iris features are extracted and the irises are matched. If the distance between them is near the decision boundary, then the structural features are extracted and combined with the statistical features to improve the accuracy of the iris recognition system. Sun et al. [27] also proposed another iris recognition system using multiple classifiers in which the local features are first used for matching irises. Again, if the distance between the irises is found to be near the decision boundary, a blob matching algorithm is then used to match the two irises. Park et al. [57] also propose a multi-algorithm approach to iris recognition. They decompose an iris image into several directional subbands using a directional filter bank (DFB) and then compute two features from these subbands, viz., binarized subband output at multiple scales and directional energy of each subband. The respective features are matched using the Hamming distance metric and the match scores of the two features are combined using a weighted sum rule resulting in a significant performance improvement.

\subsection{Fusion in other biometric systems}

Multi-instance fusion has been used to enhance the performance of various biometric recognition systems. Ross et al. [58] perform fusion of multiple instances of fingerprints at the sensor, feature and score levels. Singh et al. [59] fuse multiple instances of face image during enrolment to improve the performance of face recognition system. Lee et al. [60] use images of two fingers (multi-unit fusion) to improve the performance of fingerprint recognition system. Thus, consolidating information from multiple instance of the same biometric or from different units of that biometric enhances the performance of the biometric system.

Fusion can be done at various levels, viz., sensor level, feature level, score level, rank level and decision level [61]. Apart from the raw images and their extracted features, the match scores contains the highest amount of information. Also, they are relatively easy to access. Hence, in this chapter we mainly focus on multi-instance (multiple iris impressions) and multi-unit (using both left and right iris) information fusion in iris at the score level. Thus the goal of this chapter is two-fold:

1. Matching two enrolled impressions of the iris independently against a target iris and com- 
bining the ensuing scores using the simple sum rule (Figure 3.3 (a)).

2. Matching the enrolled left and right impressions of the iris independently against the target left and right iris, respectively, and combining the ensuing scores using the simple sum rule (Figure $3.3(\mathrm{~b}))$.

\subsection{Multi-instance iris fusion}

The WVU non-ideal iris database has a total of 207 users with $4-15$ impressions per user whereas the CASIA iris database has 108 users with 7 images per user. These irises were segmented using the Geodesic Active Contours and encoded using the multi-scale Gabor filters. The first two impressions of the iris, i.e., $I_{1}$ or $I_{2}$ of every user were treated as the enrolled iris images and the remaining impressions of that user were used as the test images. Let $S_{1}$ and $S_{2}$ denote the set of impressions from all irises corresponding to $I_{1}$ and $I_{2}$, respectively; and $Q$ denote the set of test impressions of all irises. Iris matching was done using the Hamming Distance. The performance of iris recognition by matching individual images in $S_{1}$ and $S_{2}$ against images in $Q$ for both WVU non-ideal iris database and the CASIA iris database is as shown in the Figures 3.4 and 3.5 respectively.

It is evident that the recognition performance using single iris impression of both the WVU non-ideal iris database and the CASIA database is not satisfactory. Hence, multiple impressions of the iris can be used to enhance the performance of the iris recognition system. The simplest way to combine information from multiple impressions of the same iris is by matching individual impressions in $Q$ with images in $S_{1}$ and $S_{2}$, and taking the average (sum rule) of the corresponding scores as shown in the Flowchart 3.3 (a). From the ROC curves for both the WVU non-ideal iris database and the CASIA iris database (Figures 3.6 and 3.7 respectively), it is clear that the multi-instance fusion at the score level boosts the performance of the iris recognition system. For example, for the WVU right irises, the GAR at a fixed FAR of $0.001 \%$ when matching individual images in $S_{1}$ against images in $Q$ is $61 \%$ while that of matching individual images in $S_{2}$ against images in $Q$ is $65 \%$. However, matching individual impressions in $Q$ with images in $S_{1}$ and $S_{2}$, and taking the average (sum rule) of the corresponding scores is $67 \%$. Also, for the WVU left irises, the GAR at a fixed FAR of $0.001 \%$ when matching individual images in $S_{1}$ against images in $Q$ is $59 \%$ while that of matching individual images in $S_{2}$ against images in $Q$ 


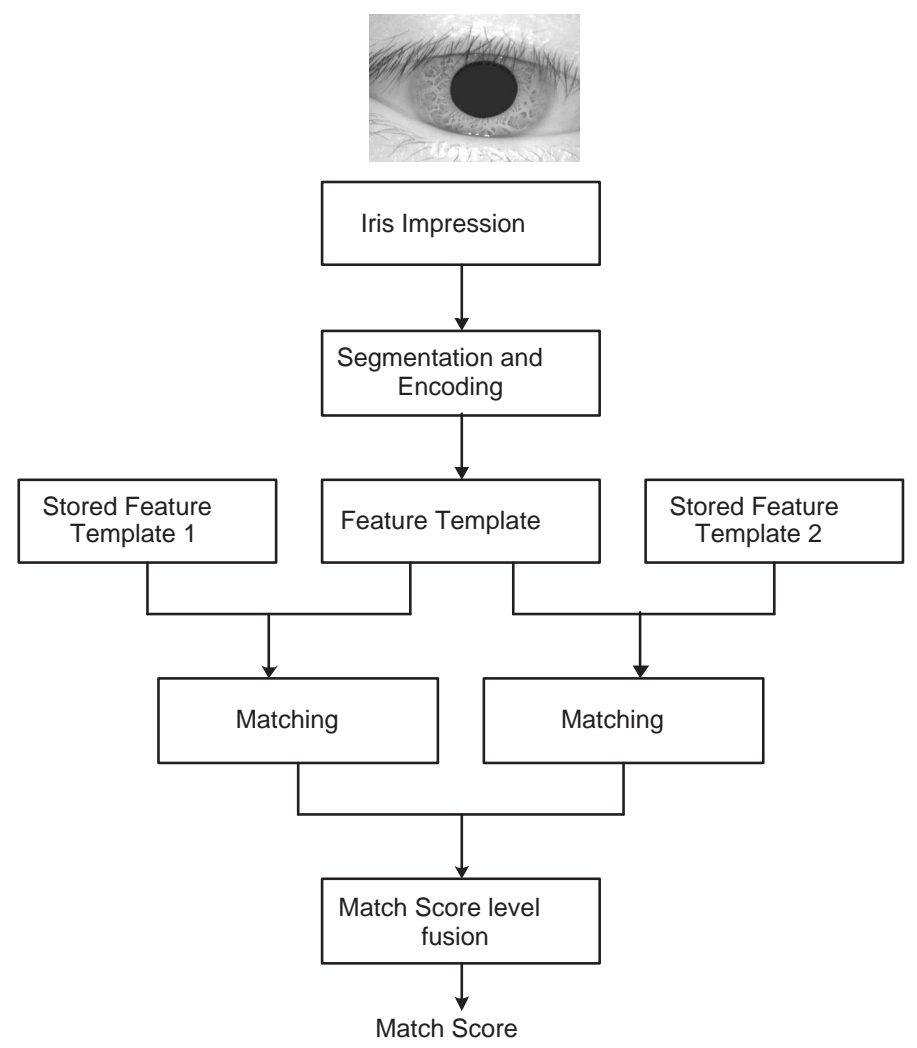

(a)

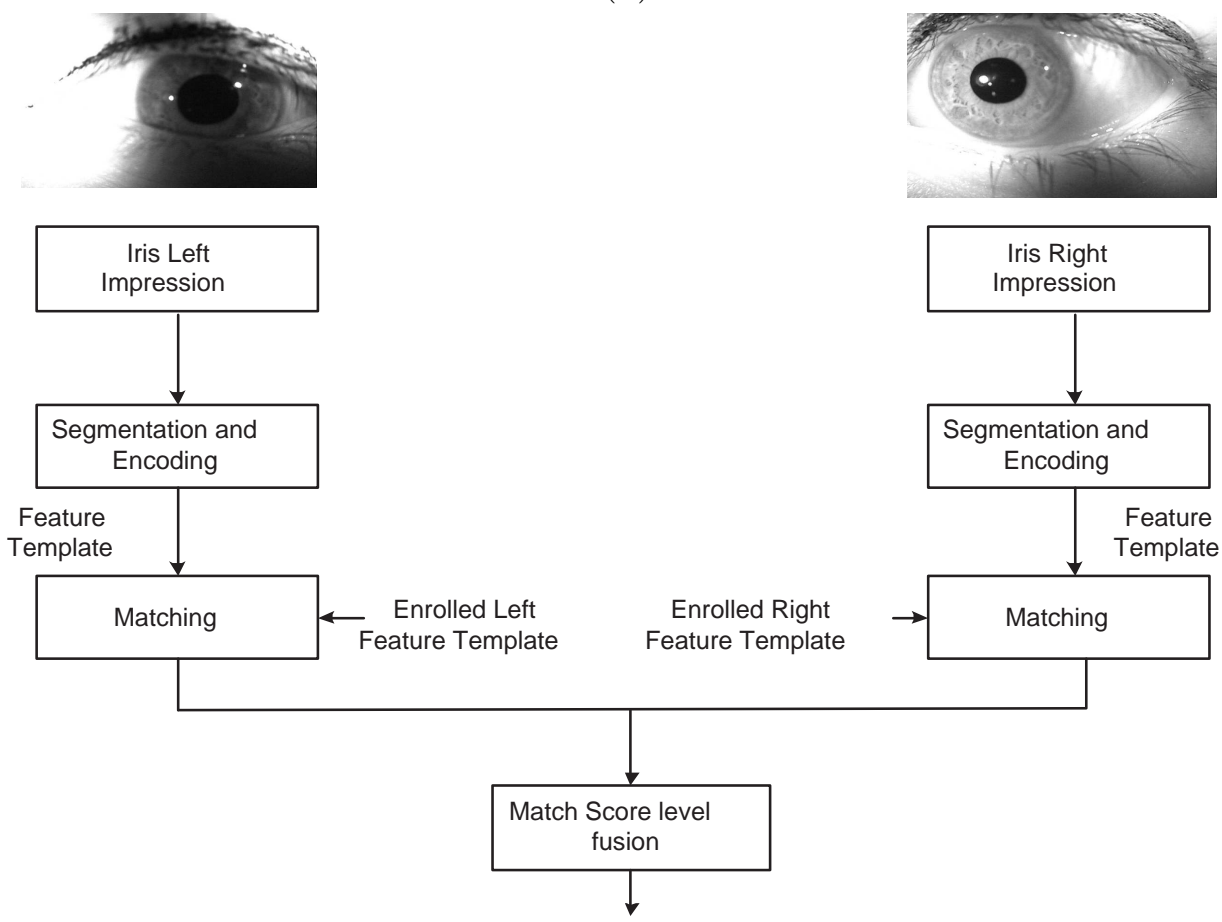

Match Score

(b)

Figure 3.3: Information fusion in irises: (a) Multi-instance fusion; (b) Multi-unit fusion. 


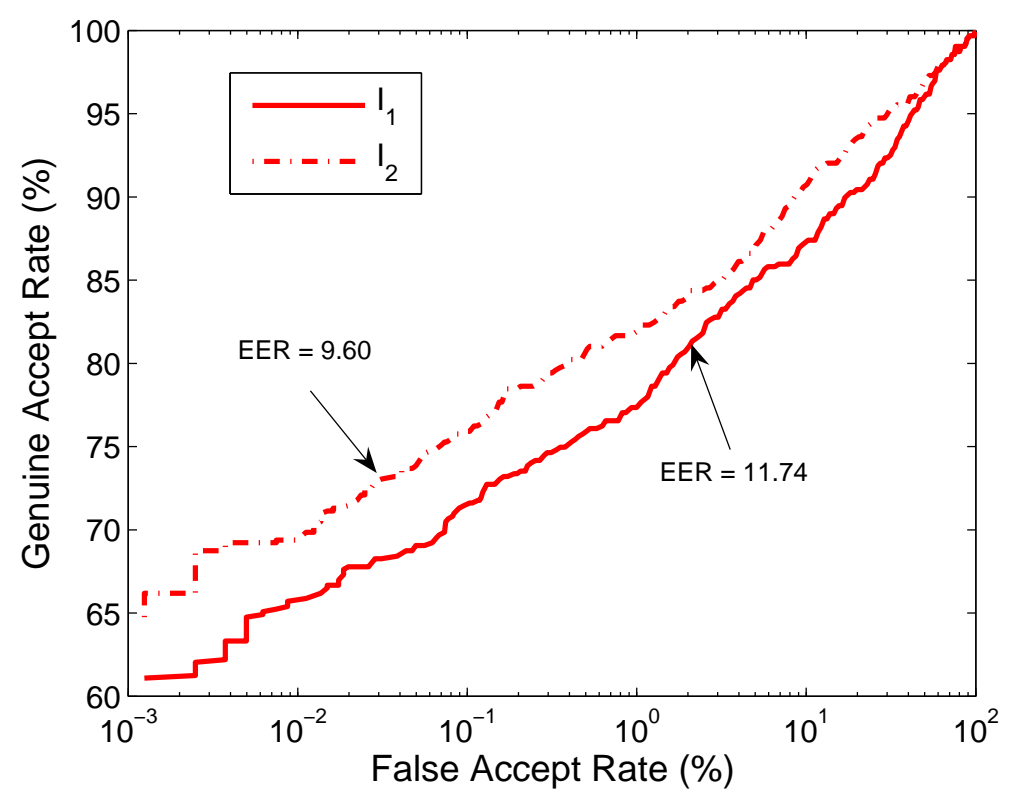

(a)

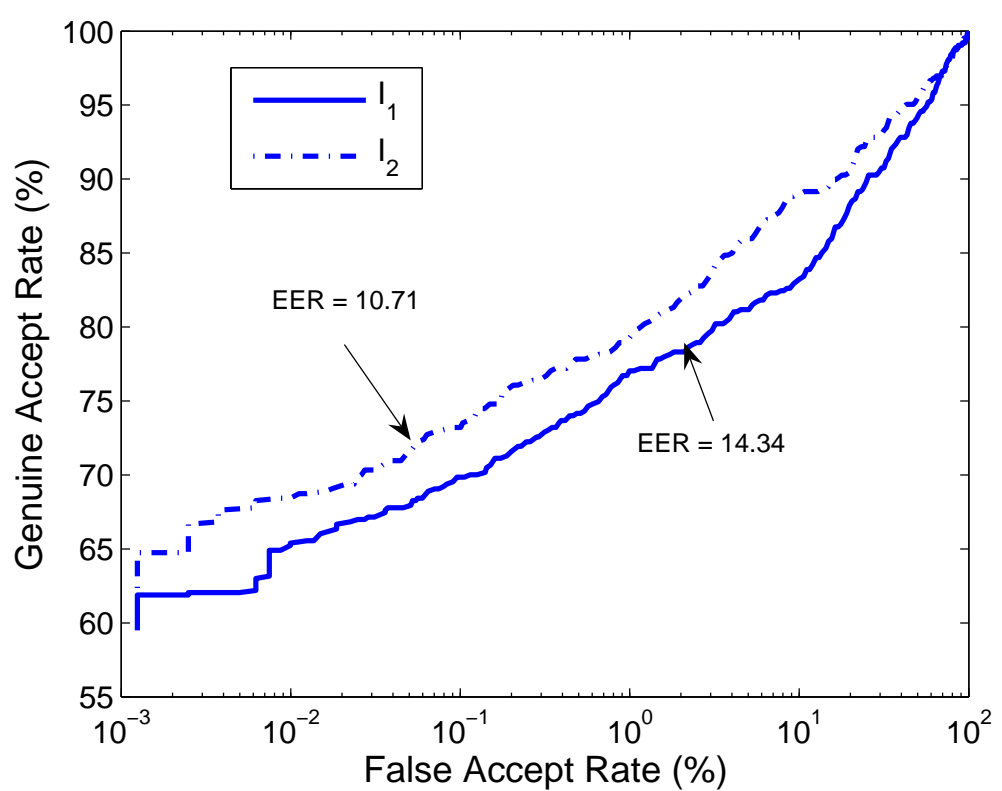

(b)

Figure 3.4: Performance of iris recognition using single enrolled impressions $\left(I_{1}\right.$ and $\left.I_{2}\right)$ of left and right irises of WVU non-ideal iris database: (a) Right iris; (b) Left iris. 


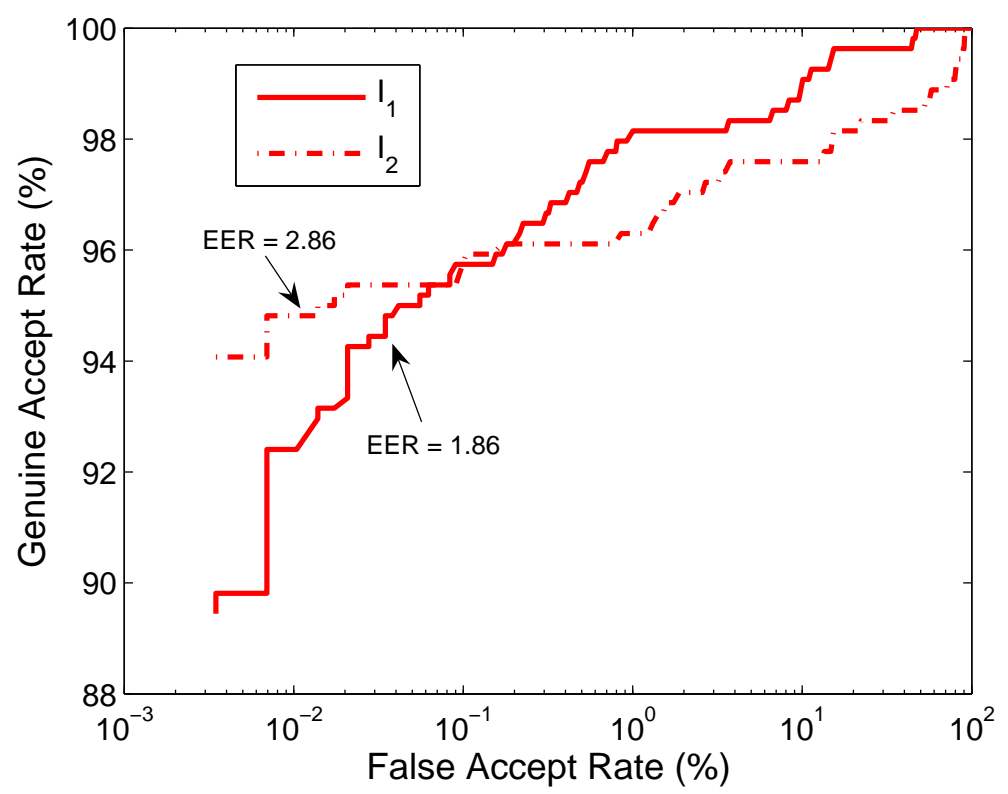

Figure 3.5: Performance of iris recognition using single enrolled impressions $\left(I_{1}\right.$ and $\left.I_{2}\right)$ of CASIA iris database.

is $62 \%$. However, matching individual impressions in $Q$ with images in $S_{1}$ and $S_{2}$, and taking the average (sum rule) of the corresponding scores is 70\%. Similarly, for the CASIA irises, the GAR at a fixed FAR of $0.01 \%$ when matching individual images in $S_{1}$ against images in $Q$ is $92.3 \%$ while that of matching individual images in $S_{2}$ against images in $Q$ is $94.6 \%$. However, matching individual impressions in $Q$ with images in $S_{1}$ and $S_{2}$, and taking the average (sum rule) of the corresponding scores is $97.2 \%$.

\subsection{Multi-unit iris fusion}

A multi-unit biometric system uses multiple units of the same body trait. For example, left and right irises, or left and right thumbs of an individual may be used to verify identity. Let $S_{L 1}$ and $S_{L 2}$ denote the set of all impressions from left irises, and $S_{R 1}$ and $S_{R 2}$ denote the set of all impressions from right irises corresponding to $I_{1}$ and $I_{2}$, respectively; Let $Q_{L}$ and $Q_{R}$ denote the set of test impressions of the left and right irises. The matching performance using a single iris unit, i.e., by matching individual impressions in $Q_{L}$ and $Q_{R}$ with images in $S_{L 1}$ (or $S_{L 2}$ ) and $S_{R 1}$ (or $S_{R 2}$ ), respectively, is poor as shown in Figure 3.4. Hence, multiple units of the iris, viz., both the left and right irises of an individual may be used to enhance the performance. Again, the 


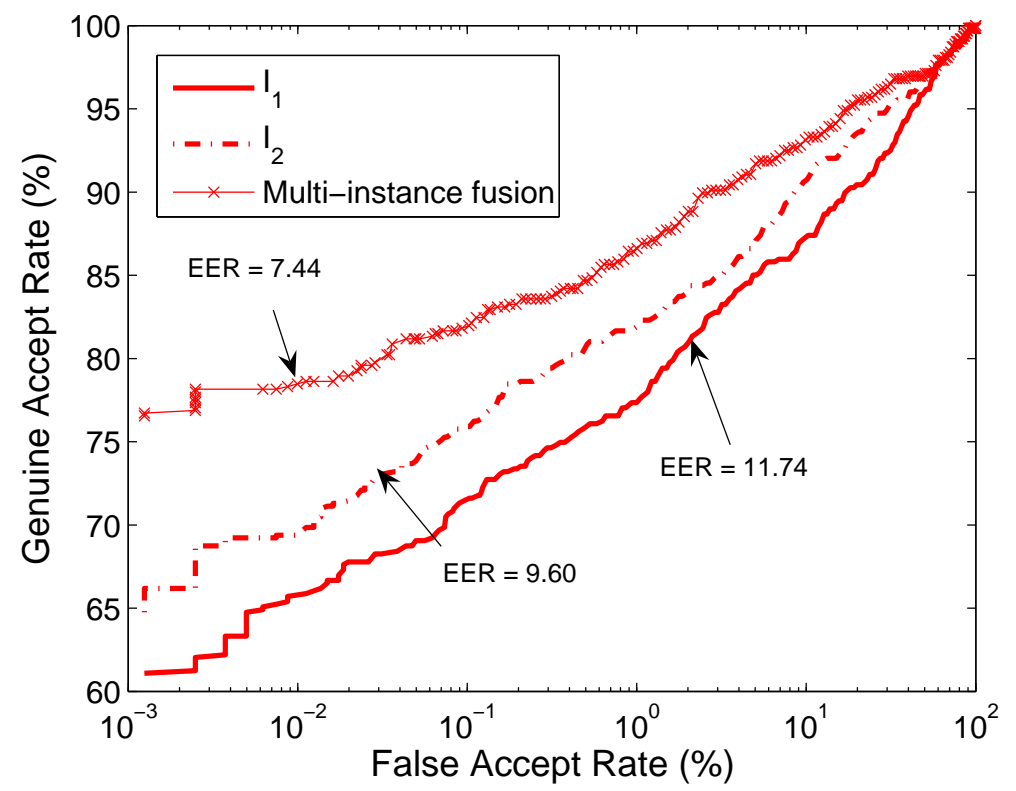

(a)

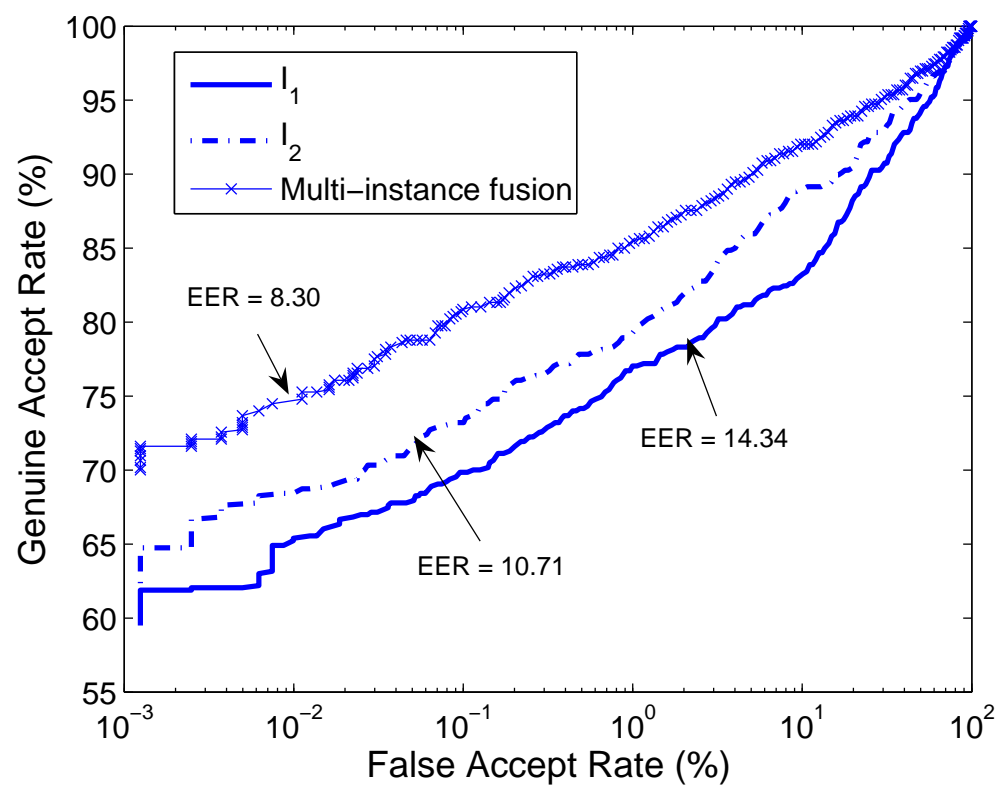

(b)

Figure 3.6: Performance improvement of iris recognition by fusing two impressions of the WVU left and right irises using the simple sum rule: (a) Right iris; (b) Left iris. 


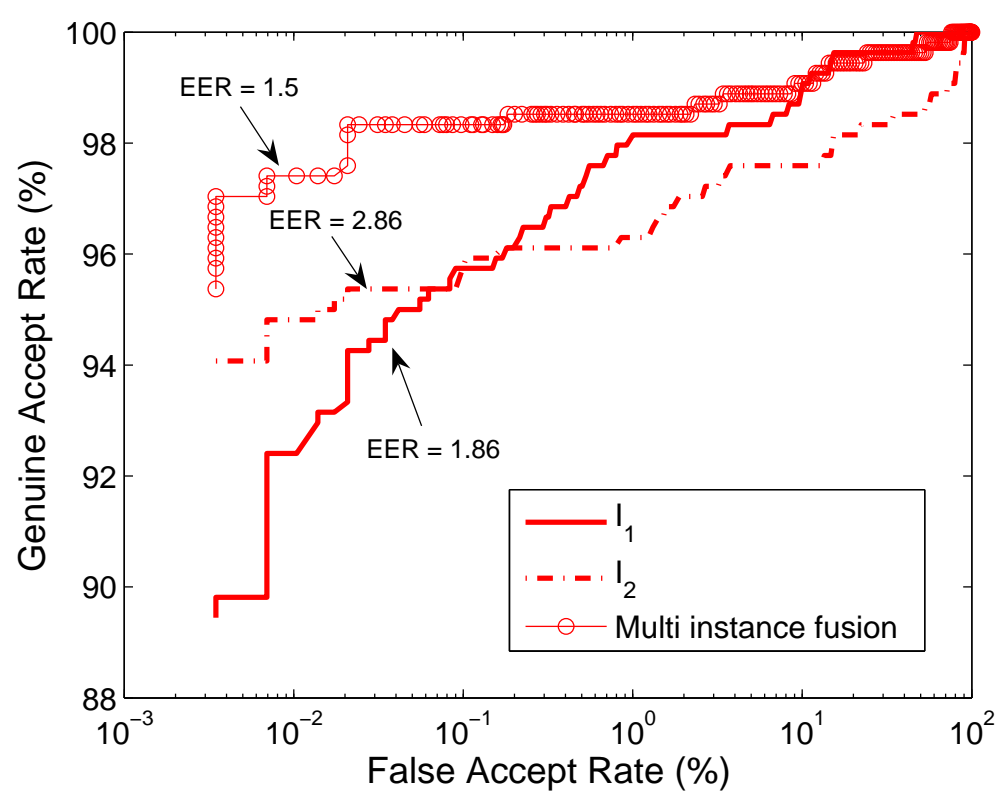

Figure 3.7: Performance improvement of iris recognition by fusing two irises of CASIA iris database using the simple sum rule.

simplest way to combine the information from both units of the iris is by matching individual impressions in $Q_{L}$ and $Q_{R}$ with images in $S_{L 1}$ (or $S_{L 2}$ ) and $S_{R 1}$ (or $S_{R 2}$ ), respectively, and taking the average (sum rule) of the corresponding scores as shown in Figure 3.3 (b). From the ROC curves in Figure 3.8, it is clear that the multi-unit fusion at the score level boosts the performance of the iris recognition system. For example, the GAR at a fixed FAR of $0.001 \%$ by matching individual images in $S_{L 1}$ against images in $Q_{L}$ is $59 \%$ while that of matching individual images in $S_{R 1}$ against images in $Q_{R}$ is $61 \%$ and that of matching individual impressions in $Q_{L}$ and $Q_{R}$ with images in $S_{L 1}$ and $S_{R 1}$, respectively, and taking the average (sum rule) of the corresponding scores is $83 \%$. Thus, multi-unit fusion in iris leads to a performance gain of more than $20 \%$.

It is evident from the ROC curves of Figures 3.6 and 3.8 that the performance gain due to multi-unit iris fusion is much more than that due to multi-unit iris fusion. This is because two impressions of the same eye will have less cumulative information compared to two impressions of left and right irises. Since there are two enrolled images $I_{1}$ and $I_{2}$ per user for both the left and right irises, another experiment which combines all the four impression (multi-instancemulti-unit) using the sum rule was conducted. Figure 3.9 shows that a significant performance gain is achieved using this approach. Thus, multi-instance-multi-unit fusion in iris leads to a performance gain of more than $30 \%$. 


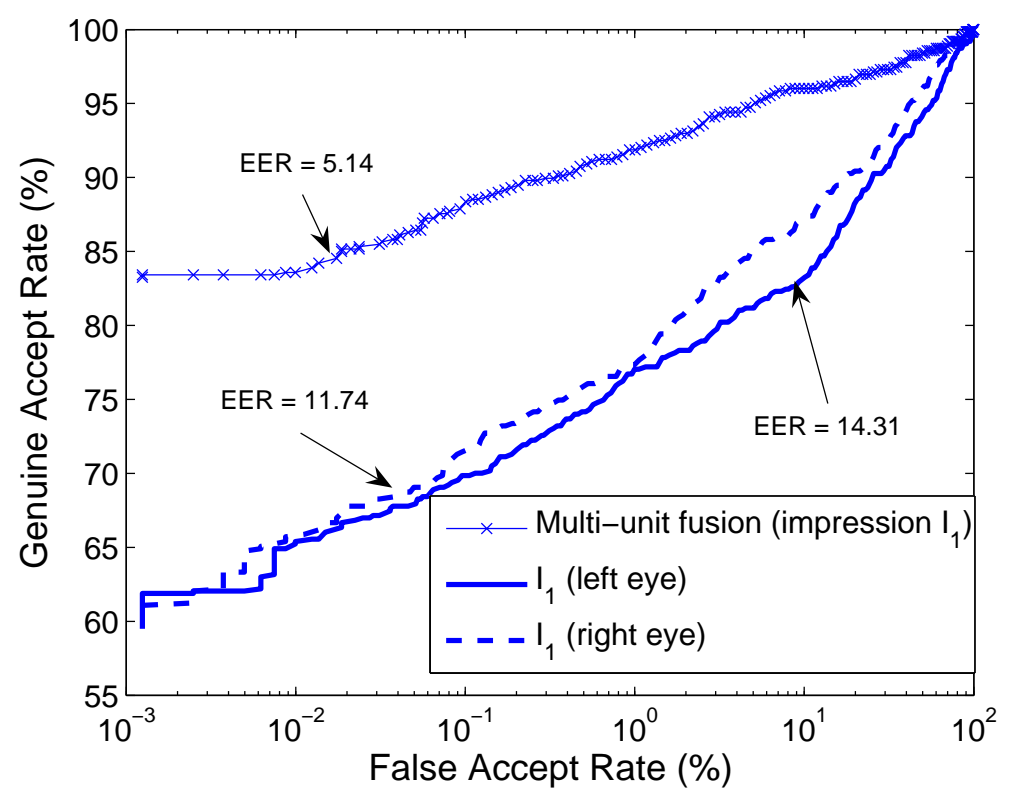

(a)

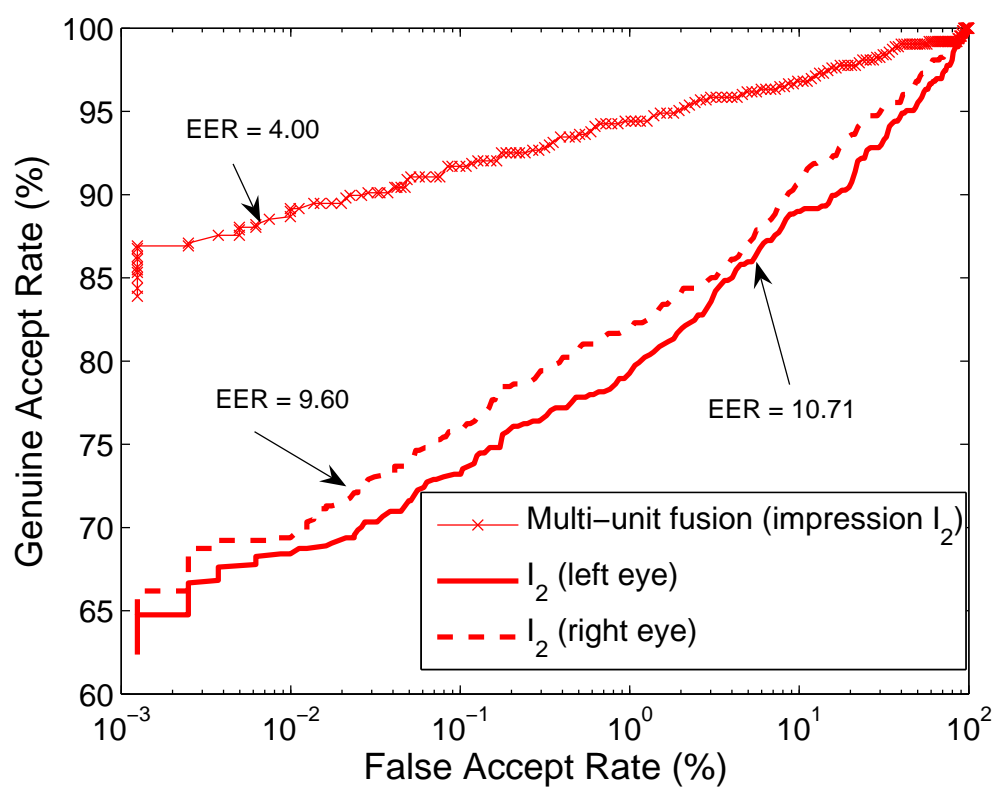

(b)

Figure 3.8: Performance improvement of iris recognition using multi-unit fusion of left and right irises using sum rule: (a) Fusing impressions $I_{1}$ from both irises; (b) Fusing impressions $I_{2}$ from both irises. 


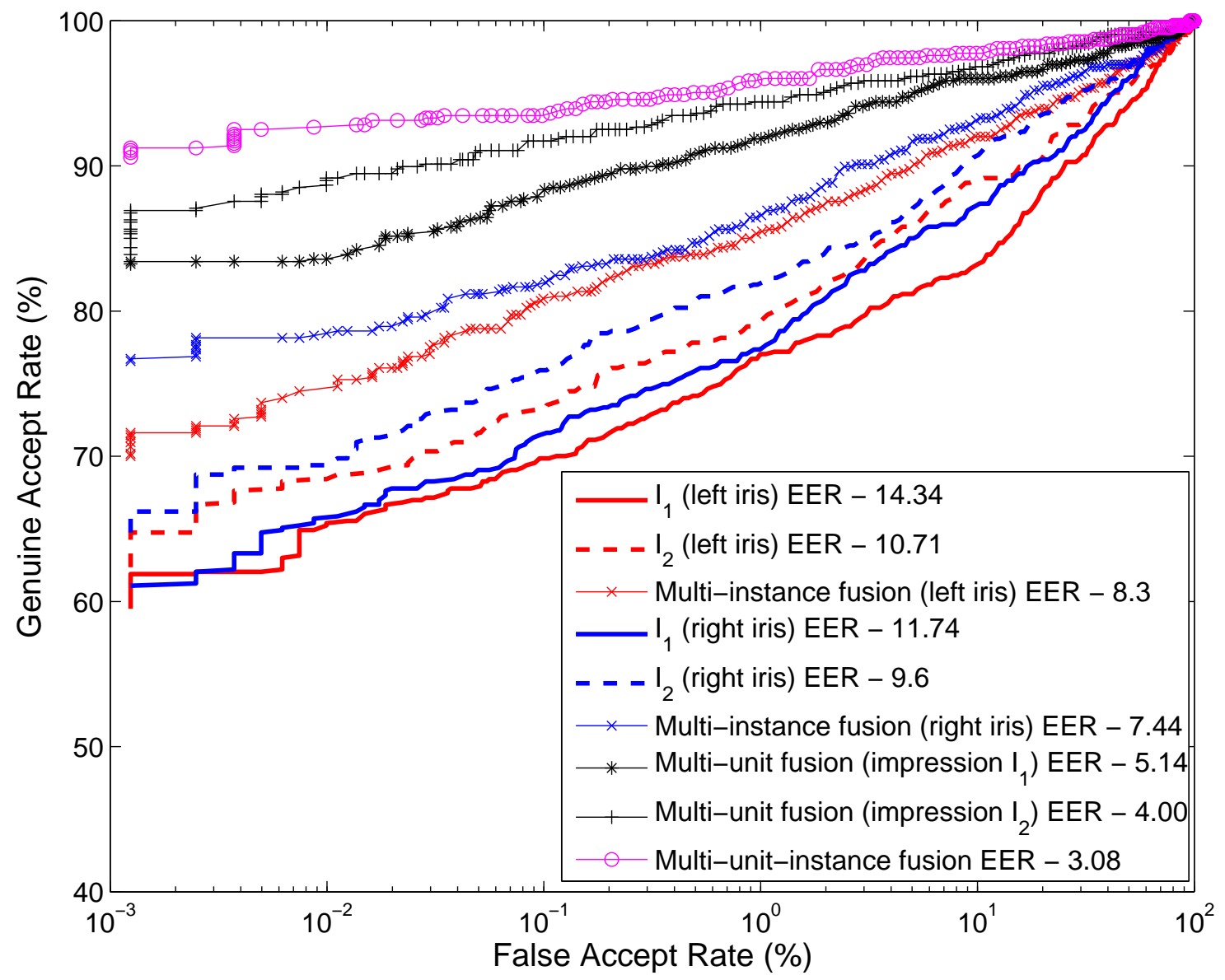

Figure 3.9: Performance improvement of iris recognition using multi-unit-instance fusion of both the $I_{1}$ and $I_{2}$ impressions of the left and right irises using the sum rule 


\begin{tabular}{|l|l|l|l|l|l|l|l|l|l|}
\hline FAR (\%) & \multicolumn{9}{|c|}{ GAR (\%) } \\
\hline & $I_{1}$ & $I_{1}$ & $I_{2}$ & $I_{2}$ & \multicolumn{2}{l|}{ Multi-instance } & Multi-unit & Multi-instance- \\
\cline { 5 - 8 } & $($ left $)$ & $($ right $)$ & $($ left $)$ & $($ right $)$ & $($ left) & (right) & $\left(I_{1}\right)$ & $\left(I_{2}\right)$ & multi-unit fusion \\
\hline 1 & 76.0 & 77.0 & 79.0 & 82.0 & 85.0 & 86.0 & 92.0 & 94.0 & 96.0 \\
\hline 0.1 & 70.0 & 72.0 & 74.0 & 76.0 & 81.0 & 82.0 & 88.0 & 92.0 & 94.0 \\
\hline 0.01 & 65.0 & 66.0 & 69.0 & 70.0 & 75.0 & 78.0 & 84.0 & 89.0 & 93.0 \\
\hline 0.001 & 59.0 & 62.0 & 63.0 & 64.0 & 70.0 & 77.0 & 84.0 & 84.5 & 91.0 \\
\hline
\end{tabular}

Table 3.1: The GAR at a fixed FAR for single impressions $I_{1}$ and $I_{2}$, multi-instance fusion $\left(I_{1}+I_{2}\right)$, multi-unit fusion ( $I_{1}$ of left $+I_{1}$ of right and similarly $I_{2}$ of left $+I_{2}$ of right) and multi-instance-multi-unit fusion.

The table 3.1 summarizes the performance improvement on the WVU non-ideal iris database by using multi-instance, multi-unit and multi-instance-multi-unit fusion.

\subsection{Discussion}

In this chapter, it has been shown that for a non-ideal iris database where image quality is an issue, multi-instance fusion of irises at the match score level using simple sum rule leads to a performance improvement in iris recognition. Also, if multiple units of the eye are used, i.e. if both the left and right iris images are used for matching, then the performance gain achieved is more than that of multi-instance fusion. Finally, if the information of both multiple units and multiple instances of irises are combined, then an approximately $30 \%$ improvement in Genuine Accept Rate is obtained at a fixed FAR of $0.001 \%$. Thus, either multi-instance or multi-unit iris fusion can be used to enhance the performance of traditional iris recognition system. 


\section{Chapter 4}

\section{Iris Synthesis using Feature}

\section{Agglomeration}

\subsection{Iris synthesis}

Many algorithms have now been proposed in the literature which use the iris as a biometric (Table 1.1). Although most existing iris recognition algorithms claim a False Accept Rate of 0\%, only Daugman's algorithm [2] has been tested on a large proprietary database containing about 799, 566 iris images [62]. There are, infact, very few publicly available iris databases. Further, the ones that are available contain data pertaining to a limited number of individuals (Table 4.1). With the emergence of several new iris recognition algorithms, ( [12] [6] [13] [14]), it is very important to have a large publicly available database to test the various algorithms being proposed. Building a large iris database is difficult because data acquisition is a time consuming process and can be expensive. Also, there are privacy issues associated with the dissemination of iris information of individuals. An alternative possibility is to develop a synthetic iris generation scheme which would permit the generation of a very large publicly available iris database that can be used to test iris algorithms.

The use of synthetic biometric database has been previously studied in the field of fingerprints. In fingerprints, Cappelli et al. [66] proposed a technique for generating synthetic fingerprints. Their software, SFINGE, has been used to develop a synthetic fingerprint image database namely DB4 for the Fingerprint Verification Competitions (FVC2000, FVC2002 and FVC2004 [67]). Three other databases (DB1, DB2 and DB3) containing real fingerprints were used in these 


\begin{tabular}{|c|c|c|c|}
\hline Database & Eyes & Images/eye & Total images \\
\hline CASIA v1.0 [63] & 108 & 7 & 756 \\
\hline UBIRIS [48] & 241 & Variable & 1877 \\
\hline UPOL [64] & 128 & 3 & 384 \\
\hline WVU off-angle & 200 & 4 & 800 \\
\hline WVU non-ideal & 414 & $4-15$ & 2678 \\
\hline ICE 2005 [65] & 132 & Variable & 2953 \\
\hline MMU [49] & 90 & 5 & 450 \\
\hline
\end{tabular}

Table 4.1: Examples of publicly available iris databases.

competitions. Performance of the fingerprint matching algorithms was analyzed on all four databases. The matching algorithms were observed to exhibit similar performance on all the four fingerprint databases. This suggested that SFINGE was able to realistically model the intra-class and inter-class variations in the fingerprints.

Similarly, in iris, Cui et al. [68] proposed an iris synthesis method by using Principal Component Analysis (PCA) and super-resolution. The eigen coefficients were first obtained from a training set of real iris images; these were modified in a controlled manner and the eigen basis were used to generate coarse synthetic iris images. These synthesized iris images were enhanced using a super-resolution scheme. Makthal and Ross [69] proposed a synthetic iris generation method using Markov Random Field (MRF) modeling. Although they generated a rich iris texture, some important features of the iris such as the radial and concentric furrows and the collarette were absent in their synthetic images. Zuo et al. [70] generated a three dimensional furrow structure and then formed a base image from furrow matrix. Their technique was predominantly dependant upon generating radial furrows. Lefohn et al. [71] created an artificial iris by stacking several semi-transparent layers where each layer consisted of one or more components of the human iris, e.g., sphincter muscle, pupil, limbus, etc.

As described in chapter 1, the iris has many features such as radial furrows, concentric furrows, crypts, collarette and the pupil. The uniqueness of an iris is not contributed by any single feature but is a combination of these multiple features. In this chapter, a novel technique for generating synthetic iris images based in feature agglomeration has been discussed. Here, the texture is first generated using a Markov Random Field (MRF)-based texture synthesis technique using a single primitive image (a 30 x 30 patch of an arbitrarily chosen iris image from the CASIA iris database). Next, different features of the iris, such as radial and concentric furrows, the collarette 
and crypts are generated on the synthetic texture. The generated iris database is validated using the following experiments: (a) Comparing the genuine and impostor distributions pertaining to the generated synthetic database and the CASIA database to verify the uniqueness of the generated synthetic iris images; (b) Clustering experiments to distinguish the iris images (both synthetic and real) from non-iris textures such as those from the Brodatz texture library. Also,

\subsection{Feature agglomeration}

Our proposed iris synthesis algorithm can be divided into two parts: (1) Texture synthesis using Markov Random Field and (2) Feature Synthesis. Both these stages are described below.

\subsubsection{Texture synthesis using Markov Random Field}

The texture of the iris is generated using a Markov Random Field (MRF) model. MRF model has been used to model a variety of textures ( [72], [73], [74], 28]) including face-like images [75]. The MRF model for images can be described as the probability distribution governing the intensity value of pixels in a specific neighborhood, also known as a clique, that is independent of all other pixels in the image. The iris texture synthesis procedure described in this chapter is based upon the work of Makthal and Ross [69] and Wei et al. [76], [77]. Here, real iris texture primitives (30 x 30 pixels) (Figure 4.1) are used to guide the synthesis process. All pixels of a randomly generated

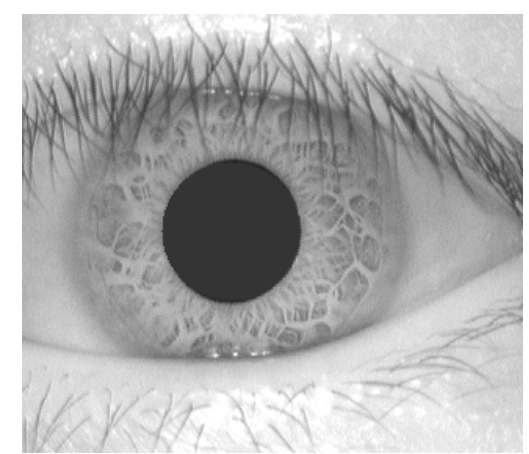

(a)

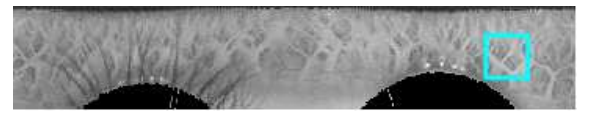

(b) (c)

Figure 4.1: Selection of primitive from a real iris image. (a) An iris image from the CASIA database; (b) The associated unwrapped image; (c) Primitive extracted from the unwrapped image.

image are iteratively updated until an iris-like texture emerges. This technique is deterministic 
as it does not use any probabilistic distribution, but rather uses neighborhood in the primitive image to compute the value of pixels in the synthetic image. The algorithm synthesizes a new texture image that is locally similar to the input primitive image but globally exhibits a different structure. The texture synthesis algorithm using a single primitive is discussed in the following sub-section.

\section{Synthesis using single primitive}

Let $P$ be the primitive image of size $30 \times 30$ pixels and let $T$ be the randomly generated white noise image. For each pixel $p$ in $T$, its neighborhood $N(p)$ is found and compared with the neighborhood of all the pixels in $P$ using the Euclidean distance. The pixel $q$ in $P$ whose neighborhood is the closest to that of $p$ is determined and its intensity is assigned to $p$. This is done repetitively for all the pixels in $T$ until the desired texture is generated. The casual neighborhood is used during synthesis and, hence, only those pixels which have already been synthesized are used for updating the pixels. To take care of the pixels on the border which do not have all their neighbors, the image is assumed to be torroidal in nature. Figure 4.2 summarizes the texture synthesis algorithm.

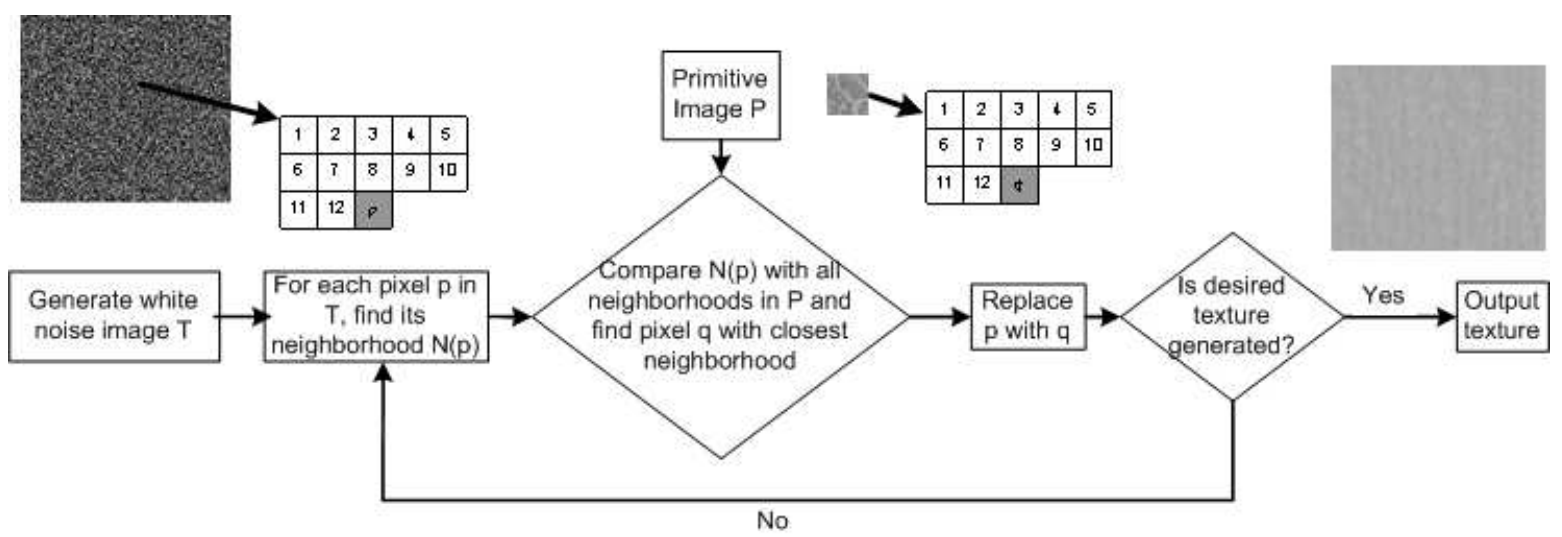

Figure 4.2: Block diagram summarizing texture synthesis using MRF.

A circular ring representing the shape of the iris is then carved out of generated texture image and the center is made darker, representing the dark color of the pupil, as shown in Figure 4.3 (c). Typically, the iris is darker in the ciliary area as compared to the pupillary area. To simulate this effect, the above synthesized iris is then subjected to a radial gradient (Figure 4.3 (d)) were the iris pixels in the vicinity of the pupil are made a little brighter than on the 


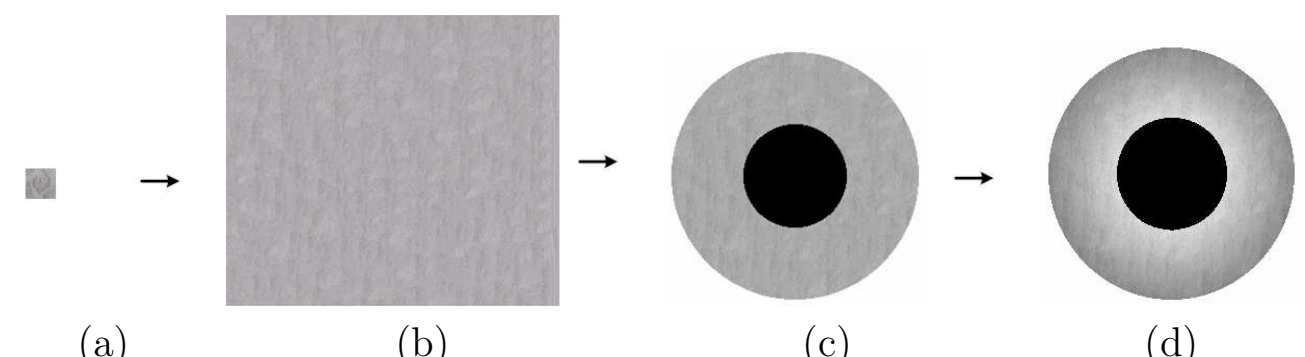

Figure 4.3: Texture synthesis using MRF. (a) Sample primitive from real iris image; (b) Bigger synthesized texture image; (c) Carved out circular iris; (d) Iris with radial gradient.

iris-sclera boundary. The intensity change between pixels near pupil-iris boundary and those on the iris-sclera boundary is made gradually.

\subsubsection{Feature synthesis}

In this chapter, the main focus is on the synthesis of iris features such as radial and concentric furrows, crypts and collarette (Figure 1.1). These features, as mentioned above, play a major role in recognizing an iris. To determine the number and position of these features, a variety of iris images in many of the publicly available iris databases for e.g. CASIA 63 were observed. Thus, the number and position of these features are parameterized based on their occurrence in real iris images. Let us consider the synthesis of these features one at a time.

(i) Radial furrows: These are the radial muscles in the iris. The contraction of these radial furrows cause pupillary dilation and thus, they control the amount of light entering the eye. They extend radially from the pupil towards the collarette. To generate them in the synthetic iris, initially straight lines are radially drawn from the center of the pupil. Each line is then sampled at discrete points and the coordinates of these points are randomly perturbed. Periodic cubic spline curves are used to interpolate through these coordinates resulting in a mesh with interlaced fibres (Figure 4.4). To impart texture to these radial furrows, Line Integral Convolution (LIC) has been used. LIC is a texture synthesis technique that is used to visualize 2-D data. LIC has been used before for imaging vector fields and producing effects such as motion blurring in images [78. Using a spatially uncorrelated gaussian noise, LIC computes the intensity values for all the coordinates of each radial furrow. It is a one dimensional filter to blur the noise image along the radial furrow resulting in a texture like appearance to the radial furrow. Consider a pixel at location $x_{0}=S\left(p_{0}\right)$ on the radial furrow. The intensity at that pixel is computed using 


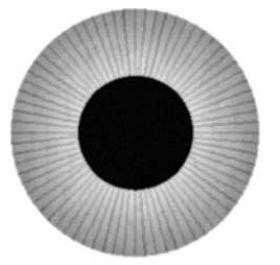

(a)

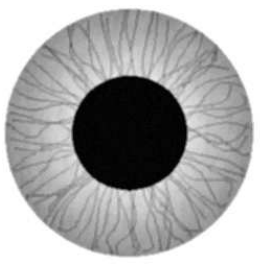

(b)

Figure 4.4: Synthesis of radial interlaced furrows. (a) Linear furrows; (b) Deformed furrows using periodic cubic splines.

one dimensional filtering as

$$
I\left(x_{0}\right)=\int_{p_{0}-L}^{p_{0}+L} k\left(p-p_{0}\right) T S(p) d p
$$

where, $T$ is a texture (gaussian noise image) and the kernel, $k$, is a one-dimensional low-pass filter with $L=25$ pixels. Thus, using LIC, texture is imparted to the radial furrows (Figure 4.5).

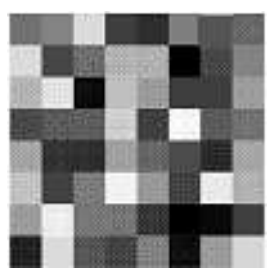

(a)

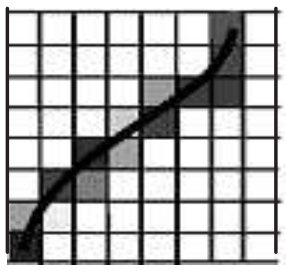

(b)

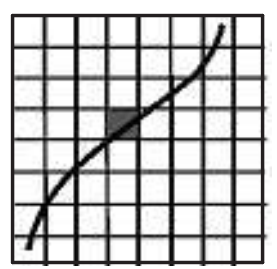

(c)

Figure 4.5: Lending texture to a streamline using LIC. (a) White noise image, T; (b) Convolving $\mathrm{T}$ with a streamline; (c) Result of LIC.

(ii) Collarette: The collarette is the boundary between the pupillary and the ciliary area. It appears as a zig-zag circumferential ridge around the pupil (Figure 1.1).

In order to create a collarette in the synthesized iris, a circle is drawn outside the pupil of radius 20 to 30 pixels greater then the pupil radius. This circle is then sampled randomly at discrete points and the coordinates of these points are randomly perturbed. Periodic cubic spline curves are used to interpolate through these coordinates resulting in a smooth zig-zag random curve representing the real shape of the collarette. All the generated radial furrows which lie within the generated curve are retained. Figure 4.6 shows the zig-zag random curve representing the collarette and also the synthesized iris containing the collarette.

(iii) Concentric Furrows: Concentric furrows are circular and concentric with the pupil. 


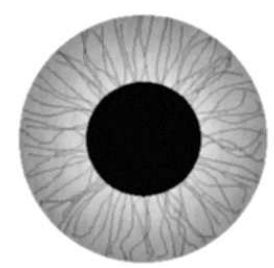

(a)

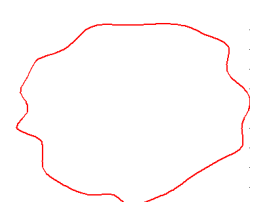

(b)

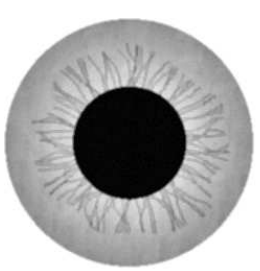

(c)

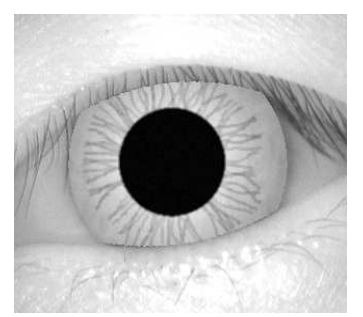

(d)

Figure 4.6: Synthesis of collarette. (a) Interlaced furrows ; (b) collarette shape generated using periodic cubic splines ; (c) Radial furrows circumscribed by collarette; (d) Synthesized iris with eyelids and eyelashes.

They typically appear in the ciliary area (Figure 1.1) and have darker color then the background texture.

To create concentric furrows, curves representing circular like shape are generated in the ciliary region with their radius 10 to 20 pixels less than the iris radius. They are made darker and blended in the background texture. Figure 4.7 shows the generated concentric furrows in the synthesized iris.

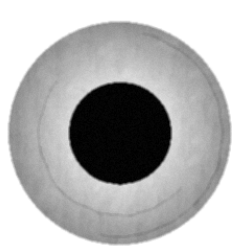

(a)

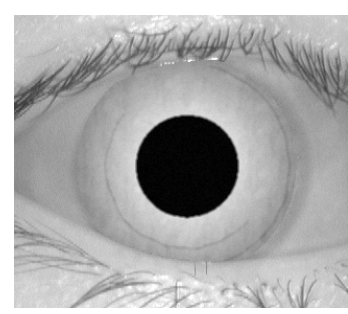

(b)

Figure 4.7: Synthesis of concentric furrows. (a) Synthesized iris with concentric furrows; (b) Synthesized iris with eyelids and eyelashes.

(iv) Crypts: Crypts are the pit-like depressions found in the iris and they typically appear near the collarette. They are the pigment related features and appear dark in color due to the dark color pigmentation of the posterior layer (Figure 1.1).

The shape and number of crypts in an iris are very random. To generate crypts, many circles (1 to 10 ) of radius 2 to 6 pixels are drawn on the periphery of the collarette. The circle is then sampled at discrete points and their coordinates are randomized. A periodic cubic spline is then used to interpolate through these points. The interpolated curve represents the actual shape of the crypts. The pixels inside the crypt are given a random value less than the value of the 
surrounding background texture. To blend the crypts in the background, the border of the crypt is found using edge detection and then pixel averaging is done along the border using a Gaussian lowpass filter. Figure 4.8 (a) and (b) show the shape of a crypt generated and its corresponding region of interest where averaging is done.

Multiple crypts are generated at random positions in the ciliary region of the iris. A Gaussian lowpass filter is applied in the region of interest (ROI) so that its intensity blends with the background texture (Figure 4.8).

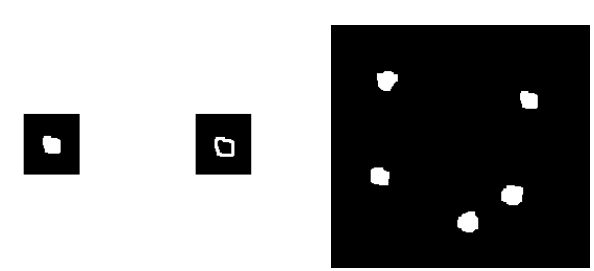

(a) (b) (c)

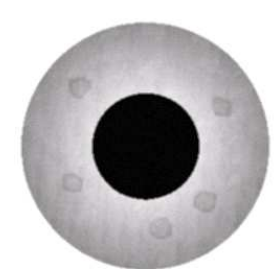

(d)

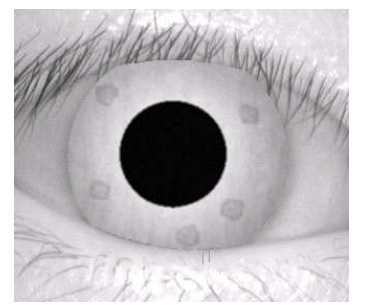

(e)

Figure 4.8: Synthesis of crypts. (a) A synthesized shape of crypt; (b) ROI for averaging; (c) Multiple crypts; (d) Synthesized iris with crypts; (e) Synthesized iris with eyelids and eyelashes.

\subsection{Results of synthesis}

The proposed synthesis scheme was used to generate a total of 1000 different classes of irises. The inter-class variability between these irises is due to the random feature synthesis and agglomeration process. Although these features are generated in a random fashion, their appearance and occurrences are parameterized based on similar features in real iris images. For example, the number of Concentric Furrows typically seen in an iris images is between 0 and 5. Hence, in our technique, the number of Concentric Furrows is randomly chosen between 0 and 5. In order to introduce intra-class variability (to obtain multiple samples for each iris), the generated synthetic irises were subjected to the following operations: (i) a random rotation in the $[-15,15]$ interval (degrees); (ii) a blurring operation using pixel averaging; (iii) addition of Gaussian noise over the entire image; and (iv) changing the pupil size by an additive factor sampled from the $[-10,10]$ interval (pixels). This resulted in 7 images per class. An example of the 7 images per class is shown in Figure 4.9 . 


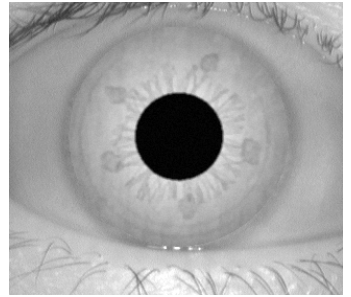

(a)

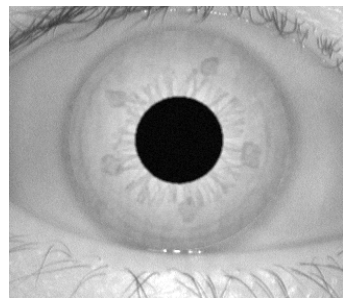

(e)

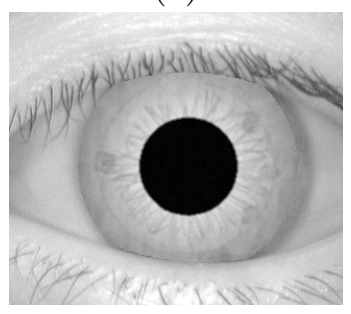

(a)

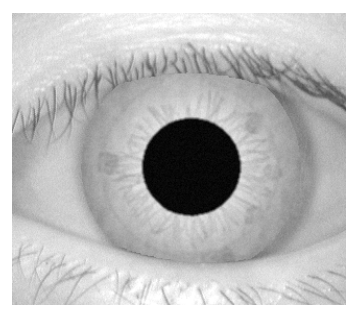

(e)

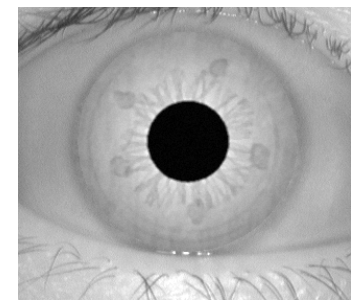

(b)

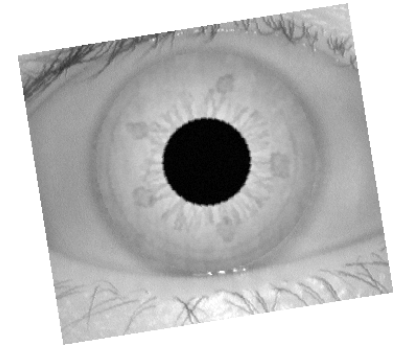

(f)

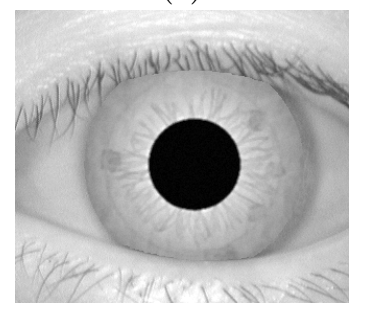

(b)

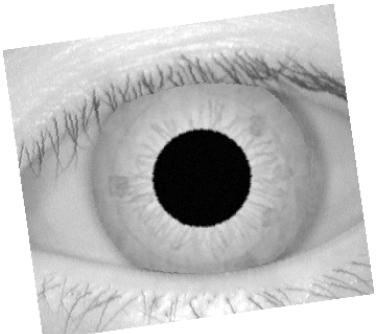

(f)

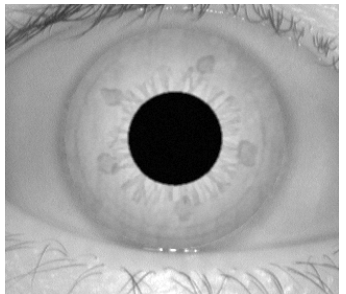

(c)

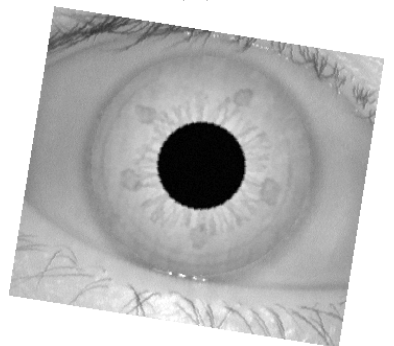

(g)

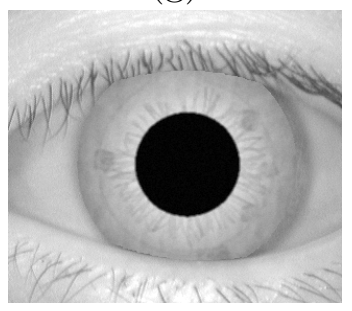

(c)

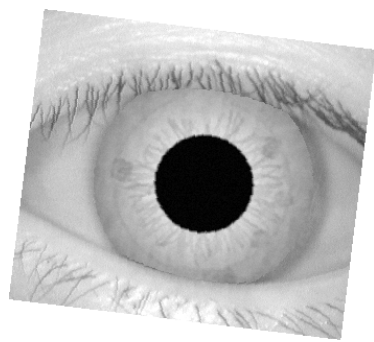

(g)

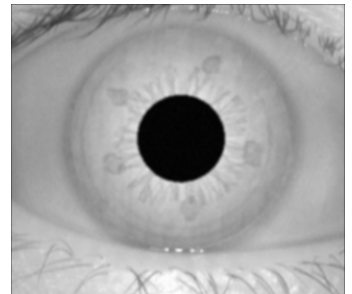

(d)

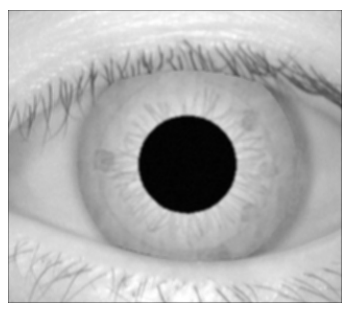

(d)

Figure 4.9: Examples of two synthesized iris classes with seven images per class. (a) Synthesized iris; (b) Pupil size decreased by 10 pixels; (c) Pupil size increased by 10 pixels; (d) Smoothed synthesized iris; (e) Synthesized iris added with noise; (f) Rotated synthesized iris (-10 degrees); (g) Rotated synthesized iris (10 degrees). 


\subsubsection{Clustering of iris images}

The synthetic iris database is created in a similar way as the real irises, rather than a deterministic way. To prove this, a simple clustering experiment using the K-means clustering method was performed to show that the generated synthetic iris images are similar to the real iris images and different than the irregular stochastic texture images from the Brodatz texture library. Cooccurrence matrix, which is used vastly in the field of texture classification and analysis, captures the second order statistics of the image. The co-occurrence matrix $C_{d}$ is of size $N * N$ where $N$ is the number of gray levels in an iris image ${ }^{11}$. Each entry of $C_{d}$ is the joint probability of a pair of pixel intensities occurring at a fixed distance $d$ apart in the image. Thus, the entry $(i, j)$ of $C_{d}$ is the probability of intensities $i$ and $j$ occurring at a distance $d=\left(d_{x}, d_{y}\right)$ apart. As there are no defined methods to select the value of $d[28$, it has been selected in increments of 5 pixels along the $\mathrm{x}$ - axis and $\mathrm{y}$ - axis respectively. A total of 111 co-occurrence matrices were considered. Various properties of the co-occurrence matrix, such as entropy, energy, contrast and homogeneity were computed. They are defined as follows:

$$
\begin{aligned}
\text { Energy } & =\sum_{i} \sum_{j} P_{d}^{2}(i, j) \\
\text { Entropy } & =-\sum_{i} \sum_{j} P_{d}(i, j) \log \left(P_{d}(i, j)\right) \\
\text { Contrast } & =\sum_{i} \sum_{j}(i-j)^{2} P_{d}^{2}(i, j) \\
\text { Homogeneity } & =\sum_{i} \sum_{j} \frac{P_{d}(i, j)}{1+|i-j|}
\end{aligned}
$$

These four properties were extracted from all the co-occurrence matrices resulting in a $111 x 4=444$ dimensional feature vector. These feature vectors were computed for $100 \mathrm{im}$ ages in the CASIA dataset, 100 images in the synthesized dataset and 100 texture images of the Brodatz library. Unsupervised K-means clustering was performed using the extracted feature vectors $(K=2)$. In this experiment, $99 \%$ of the real irises, $100 \%$ of the synthetic irises and $30 \%$ of the Brodatz images were clustered in a single class while $70 \%$ of the Brodatz textures along with $1 \%$ of the real irises were clustered in the other class. This suggests the textural similarity between the real and synthetic iris images. Table 4.2 summarizes the clustering experiment of

\footnotetext{
${ }^{1}$ Since we are using gray level iris images, $N=255$ in our experiments
} 
iris images (real and synthetic) along with the Brodatz texture images.

Table 4.2: Confusion matrix indicating iris and non-iris classification.

\begin{tabular}{|r||c|c|}
\hline \multicolumn{1}{|c||}{ True } & \multicolumn{2}{c|}{ Assigned Class } \\
\cline { 2 - 3 } Class & Iris & Non-Iris \\
\hline Real Iris & 99 & 1 \\
\hline Synthetic Iris & 100 & 0 \\
\hline Brodatz & 30 & 70 \\
\hline
\end{tabular}

\subsubsection{Comparison of genuine and impostor distributions}

In order to experiment with the synthetic images, a suitable representation and matching scheme is essential. Masek's code [18], which is a variation of Daugman's algorithm, was used to quantify and represent the textural content of the iris images. This process entails the application of a bank of Gabor filters on an iris image and examining the phase of the resulting response at regular intervals in the image. The phase response is viewed as binary information and, thus, each iris is represented using a string of 0's and 1's. The dissimilarity between two iris images is measured by employing the hamming distance metric that outputs a match score upon comparing two such binary strings. The experiments described below utilize the synthetic irises generated by the proposed method and the real irises obtained from the CASIA database 2 .

In the first experiment, three different impostor score distributions were plotted. These correspond to the impostor scores based on (a) comparing synthetic images only, (b) comparing real images only, and (c) comparing real images against synthetic images. From the distributions in Figure 4.10, it is clear that the impostor distributions are nearly the same for all the three cases. This suggests that the proposed method is able to capture the inter-class variability commonly observed in real images. In the second experiment, the genuine and impostor distributions corresponding to the real and synthetic irises were plotted (Figure 4.11). This graph also suggests that the intra-class and inter-class dynamics observed in real images are captured by the feature generation process. The genuine scores of the synthetic images were observed to be a little lower than that of real iris images. This is probably because, while generating multiple images per user, the amount of eyelid occlusion is not changed for the same user in the synthetic iris images.

\footnotetext{
${ }^{2}$ These experiments can be repeated with the other publicly available databases
} 


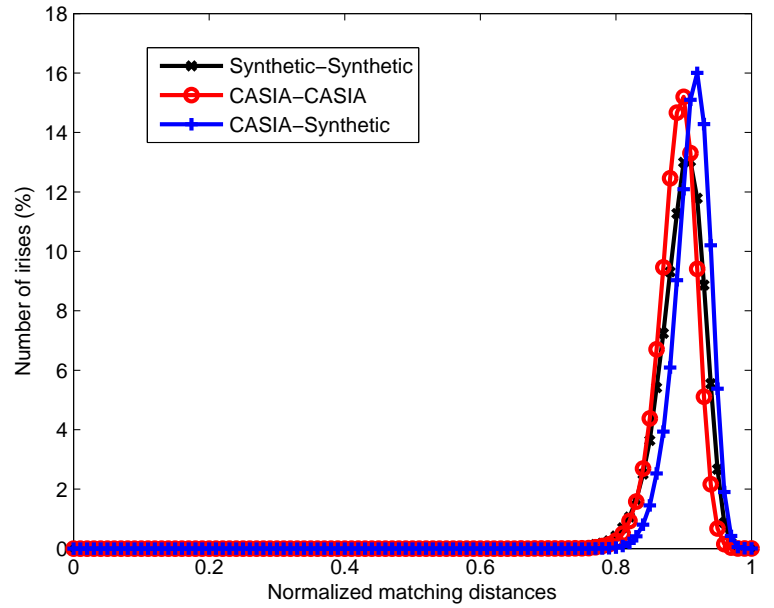

Figure 4.10: Impostor distributions for real and synthetic images.

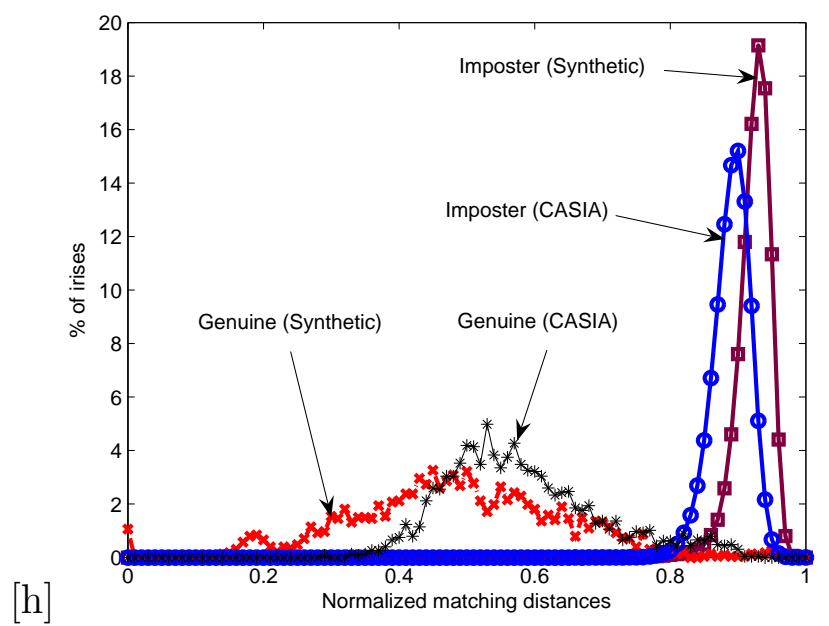

Figure 4.11: Genuine and impostor match score distributions of real and synthetic images. 


\subsection{Discussion}

In this chapter, a novel technique for generating synthetic iris images has been elaborated. Apart from synthesizing iris texture using Markov Random Fields, the proposed technique also synthesizes many iris features such as radial and concentric furrows, crypts and the collarette on the underlying texture. Makthal and Ross [69] generated a rectangular iris texture and then wrapped it to form the ring-like iris. Thus, unlike their approach which requires interpolation, in this work a circular iris structure is directly created. So the technique will not have missing points in the synthesized iris image. The synthetic irises generated using this technique are observed to be similar to the real iris images as demonstrated by the clustering experiment in which the synthetic irises are categorized alongside real iris images. Currently, the texture synthesis algorithm terminates after a predetermined number of iterations ${ }^{3}$. Hence the future work includes designing techniques to dynamically determine if the desired texture has been generated. Measures of realism that could mathematically establish the similarity between real and synthetic iris images have to be determined. Furthermore, the possibility of generating synthetic irises whose iriscode is similar to a (selected) real iris image can be examined. A few more examples of the synthesized iris images (different classes) is shown in Figure 4.12.

As a deliverable of this project, 1000 iris classes with 7 images per class were generated and distributed to some research groups and commercial organizations for research purposes. Also, a Graphical User Interface where a user can select various parameters such as number of radial fibers (between 100 and 200), number of concentric fibers (between 0 and 5), radius of the collarette, and the number of crypts (between 0 and 10) has been developed. Using these parameters, the user can generate synthetic iris images. To generate multiple iris images per class, the user can change the radius of the pupil, rotate the iris, add noise and blur the generated synthetic iris image. Figure 4.13 shows the GUI front page, parameter selection page and the result page of the GUI.

\footnotetext{
${ }^{3}$ In our experiment, texture synthesis algorithm terminates after 10 iterations
} 

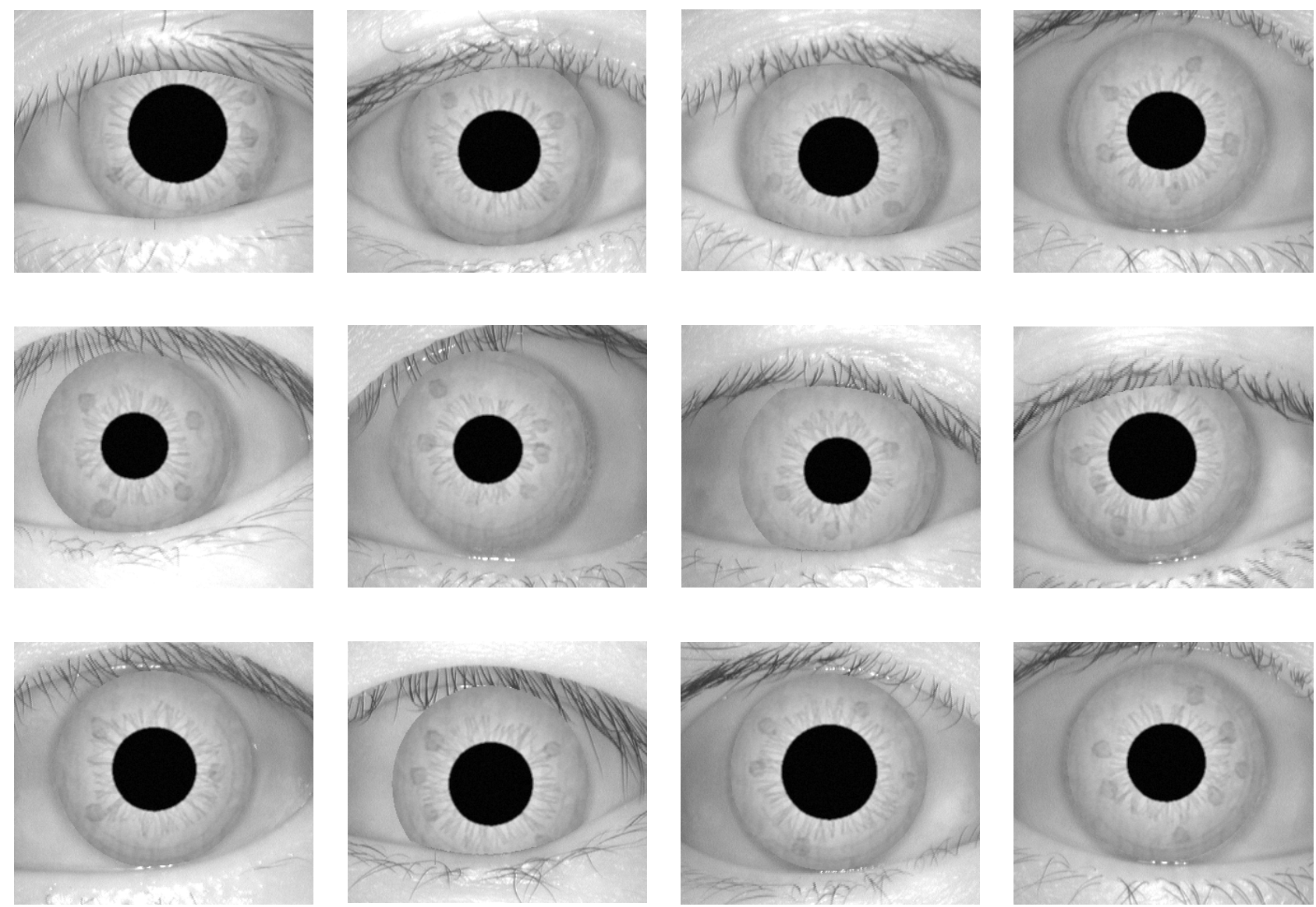

Figure 4.12: Examples of synthesized iris images of different classes. 


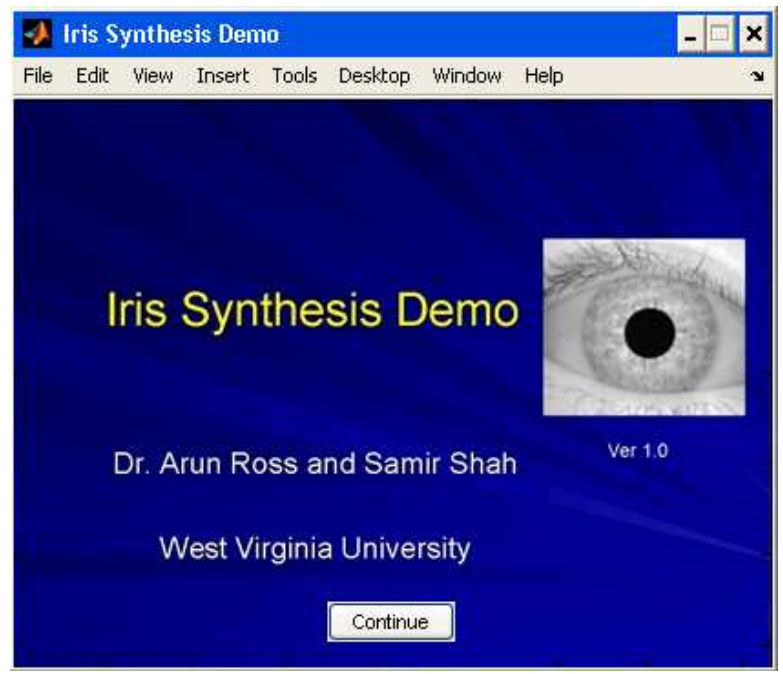

(a)

$\begin{array}{ll}\text { Radial Gradient } & \square \quad \text { LIC } \square \\ \text { Radial Furrows: } & \text { Number (0-200) } \\ \text { Colarette: } & \text { Radius (Pupil radius + 10 to Pupil radius + 35): } \\ \text { Crypts: } & \text { Number (0-10) } \\ & \text { Average Size (0-10) } \\ \text { Concentric Furrows: } & \text { Number (0-2) }\end{array}$

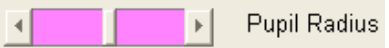

$4 \longdiv { \square }$ Smoothen $4 \sqrt{\square}$ Rotate

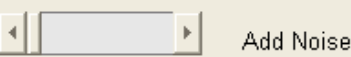

(b) 


\begin{tabular}{ll|l|} 
Radial Gradient & $\square$ & LIC $\square$ \\
Radial Furrows: & Number (0-200) & 200 \\
Colarette: & Radius (Pupil radius + 10 to Pupil radius + 35): & 60 \\
Crypts: & Number (0-10) & 5 \\
& Average Size (0-10) & 6 \\
\hline Concentric Furrows: & Number (0-2) & 1 \\
\hline
\end{tabular}
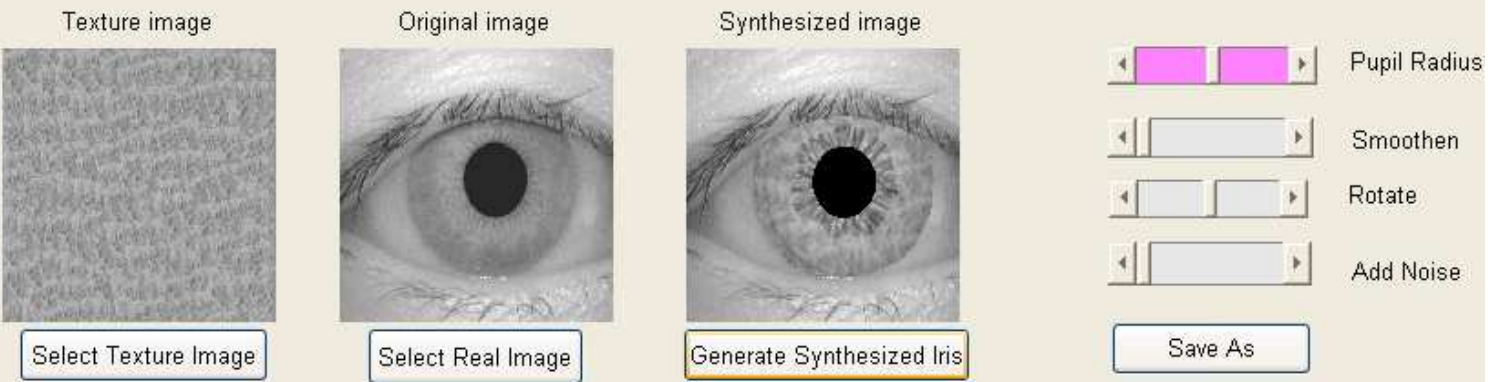

(c)

Figure 4.13: Graphical user interface. 


\section{Chapter 5}

\section{Summary and Future work}

In this thesis, a technique to enhance the performance of an iris recognition system by modifying the structure of differences in iriscodes has been presented. This novel scheme examines the block-wise structure of differences in iriscodes in order to compare two irises. This can be extremely helpful when accurate iris segmentation is not done. Experimental results on the CASIA indicate the benefits of the proposed algorithm.

The process of segmenting the iris plays a crucial role in iris recognition systems. Traditionally, systems have employed the integro-differential operator or its variants to localize the spatial extent of the iris. This thesis demonstrates a novel scheme using geodesic active contours (GAC) for iris segmentation. The GAC scheme is an evolution procedure that attempts to elicit the limbic boundary of the iris as well as the contour of the eyelid in order to isolate the iris texture from its surroundings. Experimental results on the CASIA and the WVU non-ideal dataset clearly indicate the benefits of the proposed algorithm. The algorithm also aids in accurately estimating the radius of the iris and its center. An anisotropic diffusion method was used to enhance the iris image prior to extracting its iriscode. In future, we would like to work upon improving the speed of the iris segmentation process. For example, a simple multi-resolution approach can be utilized for segmenting the iris. The evolution process can be done on a small resolution image to find the approximate position of the iris. Subsequently, the location details of the iris can be made finer by using next higher resolution image and starting the evolution from the previous coarser details of the iris. This process can be repeated until the evolution is done on the highest resolution image (input image) and thus obtaining the same level of accuracy in locating the iris but dramatically reducing the required time. The parameters in the GAC 
technique have to be tuned for each iris database separately. In future we would also like to work upon ways to generalize these parameters so that they do not need to be tuned separately for each iris database.

Multi-instance and multi-unit iris fusion systems have been evaluated in this thesis which fuse the information from two irises by using a simple score level fusion. This is particularly essential for a non-ideal iris database where the quality of the acquired irises is inferior. A significant performance improvement is observed for the WVU non-ideal iris database when the proposed fusion techniques are used. In future, an iris fusion scheme employing fusion at the feature level needs to be studied.

Also, a novel technique for generating synthetic iris images has been presented. The technique first generates background texture using MRF's and then embeds individual features of the iris such as radial and concentric furrows, crypts and the collarette on the underlying texture. The synthetic irises generated using this technique are observed to be similar to real iris images which is proven by the clustering experiment in which the synthetic irises are categorized as iris images. Currently, the synthesis algorithm terminates after predetermined number of iterations and hence we are trying to come up with a way to determine dynamically if the desired texture has been generated. We are currently working on developing measures of realism that could mathematically establish the similarity between real and synthetic iris images. Also, we would like to see if we can create a synthetic iris that can result in a nearly same "iriscode" as its primitive. 


\section{Appendix A}

\section{Iris Recognition based on Demographic}

\section{Attributes}

\section{A.1 Introduction}

As mentioned earlier, the WVU non-ideal iris database has the left and right irises of 207 individuals ${ }^{1}$. There are between $4-15$ images of both the left and right irises of each individual. The total number of images in the WVU non-ideal iris database is 2678. Each image is of size $640 \times 480$. Apart from these images, this database also has very important "meta-data" of the subjects whose iris images have been captured, viz.,

1. Ethnicity: The ethnicity of the subjects is categorized as one of the following: (a) American; (b) Asian; (c) Asian-Indian; (d) Caucasian; and (e) Other.

2. Eye color: The eye color of the subjects is categorized as one of the following: (a) Brown; (b) Black; (c) Green; (d) Blue; (e) Hazel; and (f) Other.

3. Age.

4. Sex.

5. Native Dialect.

6. Comfortable Dialect.

\footnotetext{
${ }^{1}$ This is an actively expanding database
} 
This meta data can provide very important information about the variability in iris images. It can help in answering some of these questions: How does the performance of an iris recognition system change when the color of the iris is different? Does the ethnicity of the subject affect the performance of iris recognition? How does age and sex affect it's performance? In this work, an attempt is made to study the performance of the iris recognition system for subjects with different ethnicity, eye color and sex.

\section{A.2 Experimental results}

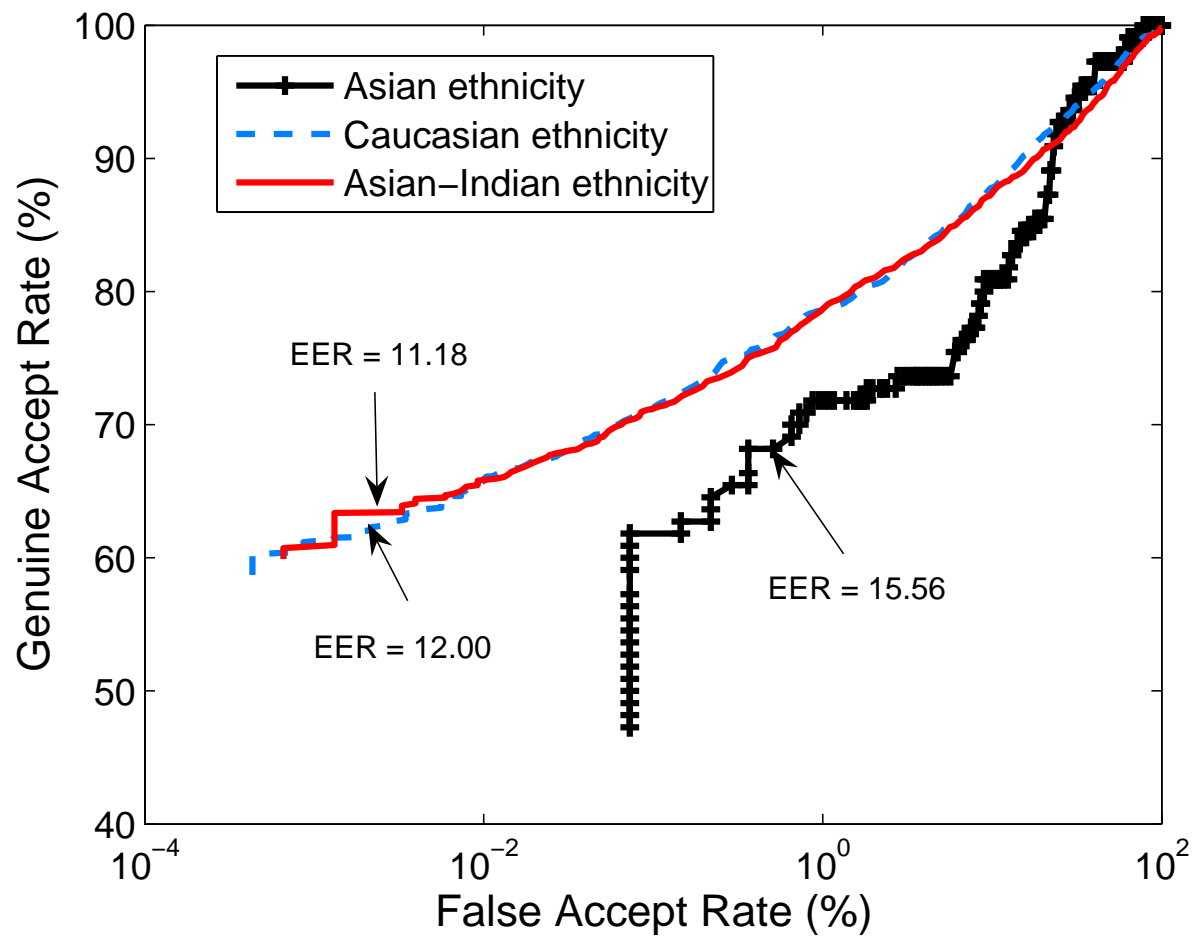

Figure A.1: Performance of iris recognition for subjects belonging to different ethnicity.

The ROC curves for subjects with different ethnicity, eye color and sex are plotted only if they are represented by at least 10 subjects. It is clear from the ROC curves in Figures A.1, A.2 and A.3 indicate that the performance of an iris recognition system may vary significantly due to these aspects. For example, the GAR at a fixed FAR of $0.1 \%$ for subjects belonging to Asian ethnicity (comprising majorly of Chinese subjects with little more occluded irises) is $45 \%$ $(E E R=15.56 \%)$, Asian-Indian ethnicity is $70 \%(E E R=11.18 \%)$ and Caucasian ethnicity is $70 \%(E E R=12.00 \%)$. The GAR at a fixed FAR of $0.01 \%$ for subjects with black iris color 


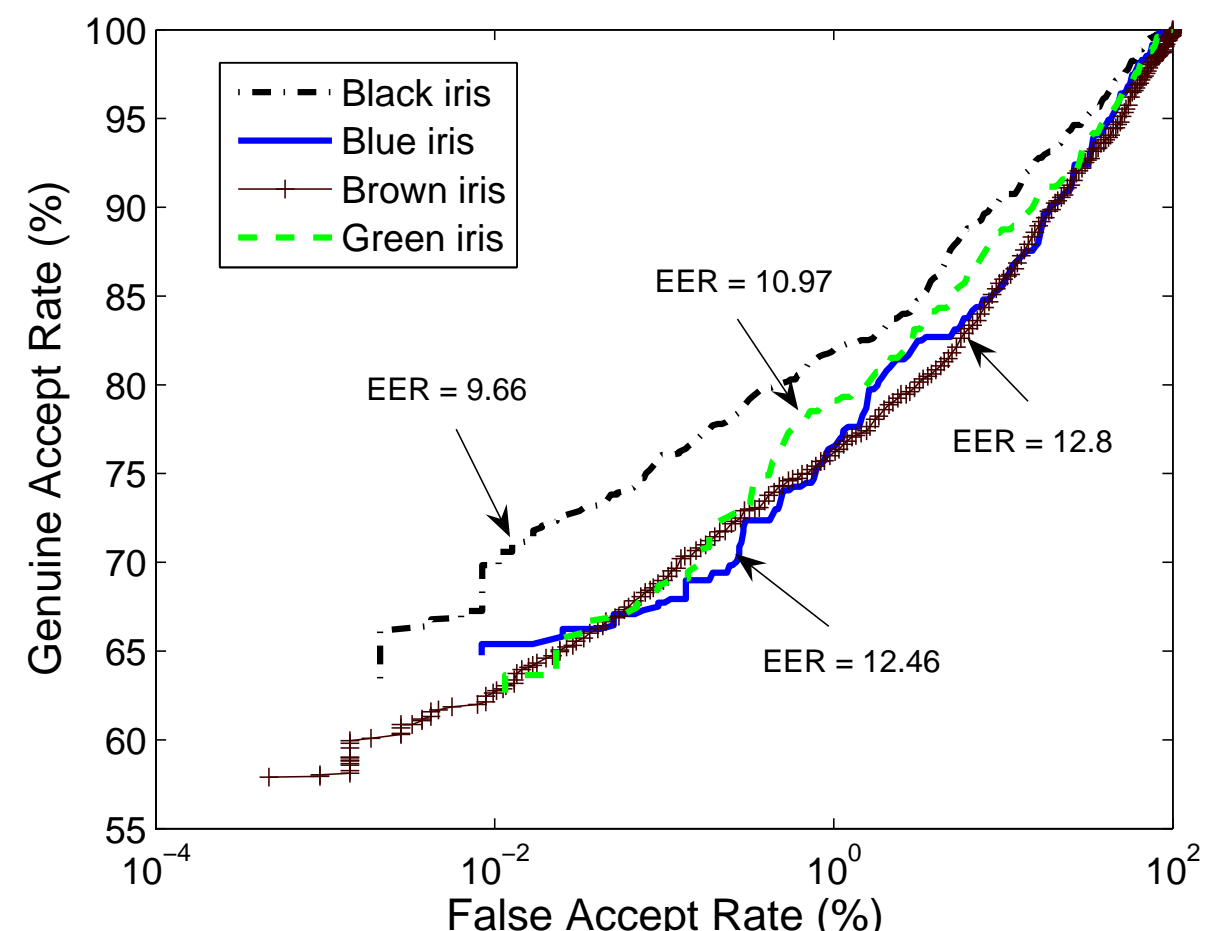

Figure A.2: Performance of iris recognition for subjects having different eye color.

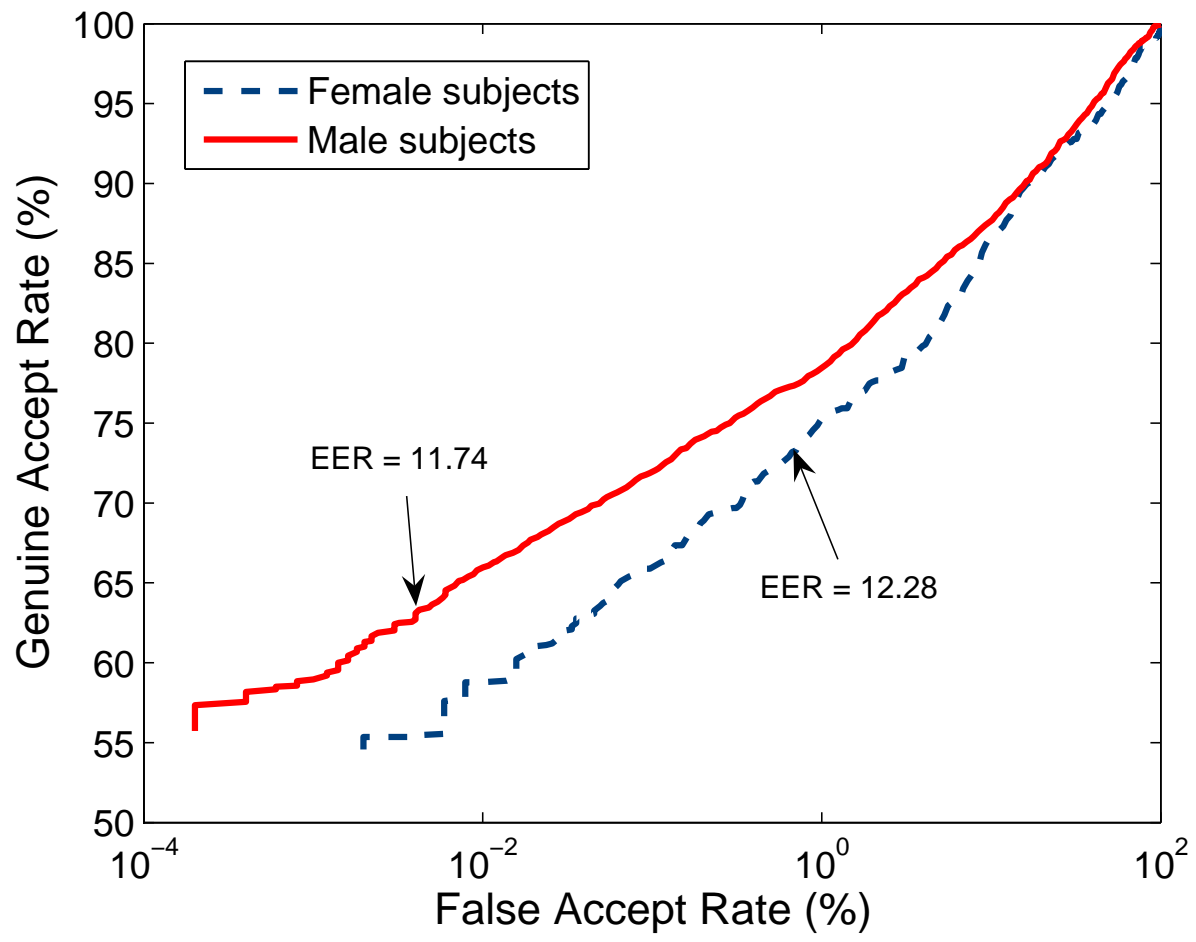

Figure A.3: Performance of iris recognition for subjects belonging to different sex. 
is $67 \%(E E R=9.66 \%)$, brown iris color is $63.0 \%(E E R=12.8 \%)$, blue iris color is $65 \%$ $(E E R=12.46 \%)$ and with green iris color is $63 \%(E E R=10.97 \%)$. The GAR at a fixed FAR of $0.01 \%$ for female subjects is $58 \%(E E R=12.28 \%)$ while for male subjects is $63 \%$ $(E E R=11.74 \%)$.

\section{A.3 Summary}

The ROC curves obtained using the "meta-data" suggest that the performance of an iris recognition system is not uniform across subjects with different ethnicity, sex and eye color. This analysis might be useful while designing an iris biometric system, for e.g., in a real world scenario like airport where subjects will be of diverse ethnicity, eye color, etc. In future, a detailed study has to be made in order to ascertain the reasons for differences in iris recognition performance for subjects with different ethnicity, eye color and sex. 


\section{References}

[1] D. Maltoni, D. Maio, A. K. Jain, and S. Prabhakar, Handbook of fingerprint recognition, 1st ed. New York, Berlin Heidelberg: Springer-Verlag, 2003.

[2] J. G. Daugman, "Demodulation by complex-valued wavelets for stochastic pattern recognition," International Journal of Wavelets, Multi-resolution and Information Processing, vol. 1, no. 1, pp. 1-17, January 2003.

[3] L. Flom and A. Safir, "Iris recognition system," U.S. Patent 4641349, February 03, 1987.

[4] J. G. Daugman, "High confidence visual recognition of persons by a test of statistical independence," IEEE Transactions on Pattern Analysis and Machine Intelligence, vol. 15, no. 11, pp. 1148-1160, November 1993.

[5] R. Wildes, J. Asmuth, G. Green, S. Hsu, R. Kolczynski, J. Matey, and S. Mcbride, "A system for automated iris recognition," in Proceedings of the Second IEEE Workshop on Applications of Computer Vision, 1994, pp. 121-128.

[6] L. Ma, T. Tan, and Y. Wang, "Efficient iris recognition by characterizing key local variations," IEEE Transactions on Image Processing, vol. 13, no. 6, pp. 739-750, June 2004.

[7] J. Daugman, "Biometric personal identification system based on iris analysis," U.S. Patent 5291 560, March 01, 1994.

[8] K. Bae, S. Noh, and J. Kim, "Iris feature extraction using independent component analysis," in Proceedings $4^{\text {th }}$ International Conference on Audio and Video Based Biometric Person Authentication (AVBPA), Guildford, UK, 2003, pp. 838-844.

[9] V. Dorairaj, N. A. Schmid, and G. Fahmy, "Performance evaluation of iris based recognition system implementing PCA and ICA encoding techniques," in Proceedings of SPIE Conference on Biometric Technology for Human Identification III, Orlando, FL, April 2005.

[10] J. G. Daugman, "Complete discrete 2-D gabor transforms by neural networks for image analysis and compression," IEEE Transactions on Acoustics, Speech, and Signal Processing, vol. 36, no. 7, pp. 1169-1179, July 1988.

[11] R. P. Wildes, "Iris recognition: An emerging biometric technology," in Proceedings of the IEEE, vol. 85, no. 9, September 1997, pp. 1348-1363.

[12] L. Ma, Y. Wang, and T. Tan, "Iris recognition using circular symmetric filters," in Proceedings of $16^{\text {th }}$ International Conference on Pattern Recognition (ICPR), vol. 2, Quebec City, Canada, August 2002, pp. 805-808. 
[13] — - "Personal identification based on iris texture analysis," IEEE Transactions on Pattern Analysis and Machine Intelligence, vol. 25, no. 12, pp. 1519-1533, December 2003.

[14] W. Boles and B. Boashash, "A human identification technique using images of the iris and wavelet transform," IEEE Transactions on Signal Processing, vol. 46, no. 4, pp. 1185-1188, April 1998.

[15] S. Lim, K. Lee, O. Byeon, and T. Kim, "Efficient iris recognition through improvement of feature vector and classifier," Journal of Electronics and Telecommunication Research Institute, vol. 33, no. 2, pp. 61-70, June 2001.

[16] A. Abhyankar and S. Schuckers, "Active shape models for effective iris segmentation," in Proceedings of SPIE Conference on Biometric Technology for Human Identification III, Orlando, FL, April 2006, p. 62020H.

[17] A. Abhyankar, L. Hornak, and S. Schuckers, "Bi-orthogonal wavelet based iris recognition," in Proceedings of SPIE Conference on Biometric Technology for Human Identification III, Orlando, FL, April 2005, pp. 59-67.

[18] L. Masek, "Recognition of human iris patterns for biometric identification," Ph.D. dissertation, The School of Computer Science and Software Engineering, The University of Western Australia, 2003.

[19] P. Kovesi, "Matlab functions for computer vision and image analysis." [Online]. Available: http://www.cs.uwa.edu.au/ pk/Research/MatlabFns/index.html

[20] J. Huang, L. Ma, Y. Wang, and T. Tan, "Iris model based on local orientation description," in Proceedings of Asian Conference on Computer Vision, Korea, April 2004, pp. 954-959.

[21] J. Huang, Y. Wang, T. Tan, and J. Cui, "A new iris segmentation method for recognition," in Proceedings of the 17th International Conference on Pattern Recognition (ICPR), vol. 3, Cambridge, England, UK, August 2004, pp. 23-26.

[22] X. Yuan and P. Shi, "Iris feature extraction using 2D phase congruency," in Third International Conference on Information Technology and Applications (ICITA), vol. 33, Sydney, Australia, July 2005, pp. 437-441.

[23] J. Thornton, M. Savvides, and B. Vijaya Kumar, "Robust iris recognition using advanced correlation techniques," in Second International Conference on Image Analysis and Recognition (ICIAR), vol. 3656. Toronto, Canada: Springer Berlin / Heidelberg, September 2005, pp. 1098-1105.

[24] D. M. Monro and D. Zhang, "An effective human iris code with low complexity," in International Conference on Image Processing (ICIP), Genova, Italy, September 2005, pp. $277-280$.

[25] C. Park, J. Lee, S. Oh, Y. Song, D. Choi, and K. Park, "Iris feature extraction and matching based on multiscale and directional image representation," in International Conference on Scale Space Methods in Computer Vision, vol. 2695, Isle of Skye, UK, June 2003, pp. 576583. 
[26] C. Park, J. Lee, M. Smith, and K. Park, "Iris-based personal authentication using a normalized directional energy feature," in Proceedings of International Conference on Audio and Video Based Biometric Person Authentication (AVBPA), Guildford, UK, June 2003, pp. 224-232.

[27] Z. Sun, T. Tan, and X. Qiu, "Graph matching iris image blocks with local binary pattern." in International Conference on Biometric Authentication (ICBA), Hong Kong, China, January 2006, pp. 366-372.

[28] L. Pau and P. Wang, The Handbook of Pattern Recognition and Computer Vision, $2^{\text {nd }}$ ed. World Scientific Publishing Co., 1998, ch. 2.1, pp. 207-248.

[29] [Online]. Available: http://www.accessexcellence.org/WN/SU/irisscan.html

[30] L. Zhukov, K. Museth, D. Breen, R. Whitaker, and A. Barr, "Level set modeling and segmentation of DT-MRI brain data," Journal of Electronic Imaging, vol. 12, no. 1, pp. 125-133, January 2003.

[31] D. Magee, A. Buliptt, and E. Berry, "Level set methods for the 3D segmentation of CT images of abdominal aortic aneurysms," in Proceedings of Medical Image Understanding and Analysis, 2001, pp. 141-144.

[32] R. Malladi, J. A. Sethian, and B. C. Vemuri, "Shape modeling with front propagation: A level set approach," IEEE Transactions on Pattern Analysis and Machine Intelligence, vol. 17, no. 2, pp. 125-133, February 1995.

[33] J. G. Daugman, "Statistical richness of visual phase information: Update on recognizing persons by iris patterns," International Journal of Computer Vision, vol. 45, no. 1, pp. 25-38, 2001.

[34] L. Masek and P. Kovesi. Matlab source code for biometric identification system based on iris patterns. The School of Computer Science and Software Engineering, The University of Western Australia. [Online]. Available: http://www.csse.uwa.edu.au/ pk/studentprojects/ libor/sourcecode.html

[35] [Online]. Available: http://www.mathworks.com/moler/leastsquares.pdf

[36] M. Bertalmio, G. Sapiro, V. Caselles, and C. Ballester, "Image inpainting," in Proceedings of the $27^{\text {th }}$ annual conference on Computer graphics and interactive techniques (SIGGRAPH 2000), New York, NY, USA, 2000, pp. 417-424.

[37] G. Emile-Male, The Restorers Handbook of Easel Painting. Van Nostrand Reinhold, New York, 1976.

[38] S. Osher and J. A. Sethian, "Fronts propagating with curvature-dependent speed: algorithms based on hamilton-jacobi formulations," Journal of Computational Physics, vol. 79, no. 1, pp. 12-49, 1988.

[39] J. A. Sethian, "A review of recent numerical algorithms for hypersurfaces moving with curvature dependent speed," Journal of Differential Geometry, vol. 31, pp. 131-161, 1989. 
[40] G. Y. Chen, Y.G. and S. Goto, "Uniqueness and existence of viscosity solutions of generalized mean curvature flow equations," Journal of Differential Geometry, vol. 33, pp. 749-786, 1991.

[41] V. Caselles, R. Kimmel, and G. Sapiro, "Geodesic active contours," International Journal of Computer Vision, vol. 22, no. 1, pp. 61-79, February/March 1997.

[42] [Online]. Available: http://csee.wvu.edu/ tmcgraw/cs593spring2006/lecture13.pdf

[43] L. D. Cohen, "On active contour models and balloons," Computer Vision, Graphics, Image Processing: Image Understanding, vol. 53, no. 2, pp. 211-218, 1991.

[44] J. Sethian and J. Strain, "Crystal growth and dendritic solidification," Journal of Computational Physics, vol. 98, pp. 231-253, 1992.

[45] F. L. Bookstein, "Principal warps: Thin-plate splines and the decomposition of deformations," IEEE Transactions on Pattern Analysis and Machine Intelligence, vol. 11, no. 6, pp. 567-585, June 1989.

[46] M. Kass, A. Witkin, and D. Terzopoulos, "Snakes: Active contour models," International Journal of Computer Vision, vol. 1, no. 4, pp. 321-331, 1987.

[47] P. Perona and J. Malik, "Scale-space and edge detection using anisotropic diffusion," IEEE Transactions on Pattern Analysis and Machine Intelligence, vol. 12, no. 7, pp. 629-639, July 1990.

[48] H. Proena and L. A. Alexandre, "UBIRIS: A noisy iris image database," in Proceedings of International Conference on Image Analysis and Processing (ICIAP), vol. 1, 2005, pp. 970-977. [Online]. Available: http://iris.di.ubi.pt

[49] “The MMU1 iris image database." [Online]. Available: http://pesona.mmu.edu.my/ ccteo/

[50] N. Kalka, J. Zuo, V. Dorairaj, and N. Schmid, "Image quality assessment for iris biometric," in Proceedings of SPIE Conference on Biometric Technology for Human Identification, vol. 6202, April 2006, pp. 61020D-1-61 020D-11.

[51] Y. Chen, S. Dass, and A. K. Jain, "Localized iris image quality using 2-d wavelets," in International Conference on Advances in Biometrics (ICB), vol. 3832, Hong Kong, China, January 2006, pp. 373-381.

[52] International Biometric Group, "Independent testing of iris recognition technology (ITIRT) - Final Report," May 2005.

[53] J. Jang, K. Park, J. Son, and Y. Lee, "A study on multi-unit iris recognition," in Proceedings of the $8^{\text {th }}$ conference on Control, Automation, Robotics and Vision (ICARCV), vol. 2, Kunming, China, December 2004, pp. 1244-1249.

[54] — - "Multi-unit iris recognition system by image check algorithm," in International Conference on Biometric Authentication (ICBA), vol. 3072, Hong Kong, China, 2004, pp. 450457.

[55] [Online]. Available: ftp://ftp.panasonic.com/pub/Panasonic/CCTV/SpecSheets/ BM-ET300.pdf 
[56] Z. Sun, Y. Wang, T. Tan, and J. Cui, "Cascading statistical and structural classifiers for iris recognition." in International Conference on Image Processing (ICIP), Singapore, October 2004, pp. 1261-1264.

[57] C. Park and J. Lee, "Extracting and combining multimodal directional iris features," in International Conference on Biometric Authentication (ICBA), vol. 3832, Hong Kong, China, January 2006, pp. 389-396.

[58] A. Ross, S. Shah, and J. Shah, "Image versus feature mosaicing: A case study in fingerprints," in Proc. of SPIE Conference on Biometric Technology for Human Identification III, Orlando, FL, April 2006, p. 620208.

[59] R. Singh, M. Vatsa, A. Ross, and A. Noore, "Performance enhancement of $2 \mathrm{~d}$ face recognition via mosaicing," Buffalo, USA, October 2005, pp. 63-68.

[60] K. Lee, K. Park, S. Lee, and J. Kim, "Multi-unit biometric fusion in fingerprint verification." in International Conference on Biometric Authentication (ICBA), Hong Kong, China, July 2004, pp. 395-402.

[61] A. Ross, K. Nandakumar, and A. Jain, Handbook of Multibiometrics (International Series on Biometrics). Secaucus, NJ, USA: Springer-Verlag New York, Inc., 2006.

[62] M. ALMualla, "The UAE iris expellees tracking and border control system," Biometric Consortium, Crystal City, VA, September 2005.

[63] "The CASIA iris image database." [Online]. Available: http://www.sinobiometrics.com

[64] M. Dobes and L. Machala, "UPOL iris image database." http://phoenix.inf.upol.cz/iris/, 2004.

[65] [Online]. Available: http://iris.nist.gov/ice/

[66] R. Cappelli, A. Erol, D. Maio, and D. Maltoni, "Synthetic fingerprint-image generation," in Proceedings of the $15^{\text {th }}$ International Conference on Pattern Recognition (ICPR), vol. 3, Barcelona, Spain, September 2000, pp. 475-478.

[67] "University of bologna: Biometric systems laboratory." [Online]. Available: http: //bias.csr.unibo.it/research/biolab/sfinge.html

[68] J. Cui, Y. Wang, J. Huang, T. Tan, and Z. Sun, "An iris image synthesis method based on PCA and super-resolution," in Proceedings of the $17^{\text {th }}$ International Conference on Pattern Recognition (ICPR), vol. 4, Cambridge, England, UK, August 2004, pp. 471-474.

[69] S. Makhtal and A. Ross, "Synthesis of iris images using markov random fields," in Proceedings of 13th European Signal Processing Conference (EUSIPCO), Antalya, Turkey, September 2005 .

[70] J. Zuo and N. Schmid, "Generation of synthetic irises," in Proceedings of the Biometrics Consortium Conference (BCC), Crystal City, VA, September 2005.

[71] A. Lefohn, B. Budge, P. Shirley, R. Caruso, and E. Reinhard, "An ocularist's approach to human iris synthesis," IEEE Computer Graphics and Applications, vol. 23, no. 6, pp. 70-75, November/December 2003. 
[72] A. K. Jain and G. R. Cross, "Markov random field texture models," IEEE Transactions on Pattern Analysis and Machine Intelligence, vol. 5, no. 1, pp. 25-39, 1983.

[73] R. Paget, "Strong markov random field," IEEE Transactions on Pattern Analysis and Machine Intelligence, vol. 26, no. 3, pp. 408-413, March 2004.

[74] S. Z. Li, Markov Random Field Modeling in Image Analysis. Springer-Verlag, 2001.

[75] A. K. Jain, S. C. Dass, and X. Lu, "Face detection and synthesis using markov random field models," in Proceedings of International Conference on Pattern Recognition (ICPR), Quebec City, Canada, August 2002, pp. 201-204.

[76] L. Y. Wei, "Texture synthesis by fixed neighborhood searching," Ph.D. dissertation, Stanford University, 2001.

[77] L. Y. Wei and M. Levoy, "Fast texture synthesis using tree-structured vector quantization," in Proceedings of the 27th Annual Conference on Computer Graphics and Interactive Techniques, New Orleans, LA, USA, July 2000, pp. 479-488.

[78] B. Cabral and L. Leedom, "Imaging vector fields using line integral convolution," in Proceedings of the 20th Annual Conference on Computer Graphics and Interactive Techniques, Anaheim, CA, USA, 1993, pp. 263-270. 